\title{
A methodology for optimal design of the Canadian SCWR reactor core
}

\author{
by \\ Mohammadhossein Nimrouzi
}

A thesis submitted to the Faculty of Graduate Studies and Research in partial fulfillment of the requirements for the degree of

Master of Applied Science in Mechanical and Aerospace Engineering Carleton University

Ottawa, Ontario, Canada, K1S 5B6

(c) Copyright 2014, Mohammadhossein Nimrouzi 
The undersigned recommend to

the Faculty of Graduate and Postdoctoral Affairs

acceptance of the thesis

\title{
A methodology for optimal design of the Canadian SCWR reactor core
}

\author{
submitted by \\ Mohammadhossein Nimrouzi, B.A.Sc. \\ in partial fulfillment of the requirements for \\ the degree of Master of Applied Science in Mechanical and Aerospace Engineering \\ Carleton University
}

Thesis Supervisor, John A Goldak

Thesis Co-supervisor, Dr. Metin Yetisir. AECL

Metin Yaras, Chair, Department of Mechanical and Aerospace Engineering

Carleton University

April 2014 


\section{Abstract}

The goal of this thesis is to demonstrate a capability to do design by analysis for pressure vessels, i.e., design based on a 3D nonlinear coupled holistic macroscopic analyses.

This thesis represents a robust methodology for optimization of the GEN IV reactor core that is time-efficient and reasonably capable to do accurate nonlinear thermal/stress analysis. The optimized design of Canadian SCWR provided by parameterization of different parts of the plenum are presented. A parametric mesh has three important advantages. The parameters can be used as design variables to optimize the design using Design of Experiment and optimization. Because a parametric mesh can be created automatically by a computer program, the user does not need to create new meshes to explore a design space. Because the parametric meshes are created automatically, time is minimized and the risk of human error is reduced Moreover, the sensitivity of the defined objective functions to each mesh parameter was computed.

Also, the optimized profile of temperature and pressure for start up and shut down cycles was investigated with respect to an objective function to minimize the effective plastic strain. The optimized value of effective plastic strain for the case that pressure is a function of saturated temperature was determined.

The capability of $\operatorname{VrSuite}^{1}$ to capture the shake down behavior of a structure by using the Bree test was verified. Using the described analysis methodology, the need

\footnotetext{
${ }^{1}$ www.Goldak-VrWeld.com.
} 
for optimizing the design to tolerate a fatigue load of repeated start up shut down cycles was provided and possible ways to improve the design were suggested. 


\section{Acknowledgments}

I wish to express my deepest gratitude to Prof. John A Goldak, my supervisor, for many suggestions and constant supports during this thesis work. I would have been lost without them. His tireless and detailed involvement in my research has been an invaluable asset for me. I would also like to sincerely thank my co-supervisor, Dr. Metin Yetisir for his support, guidance, encouragement and knowledge.

I would also like to extend a heartfelt thanks to Goldak Technologies Inc, Mr. Dan Downey, Mr. Stanislav Tchernov and Mr. Jianguo Zhou, for all their efforts in the research group to provide a better research environment and all their kindness during the period that I was working on this project.

I would like to thank Natural Resources Canada (NRCan) and the National Sciences and Engineering Research Council (NSERC) for their support of the Canadian Generation IV National Program.

Furthermore, I would also like to thank Mr. Michel Gaudet and the Atomic Energy of Canada Ltd (AECL) for all their technical supports and providing information. 
To my parents 


\section{Contents}

1 Introduction 1

1.1 Background ........................... 1

1.2 Scope and Organization of the Thesis . . . . . . . . . . . . . . 3

2 Verification of Bree test with VrSuite $\quad 6$

2.1 Introduction . . . . . . . . . . . . . . . . 6

2.2 Definition of Bree problem . . . . . . . . . . . . . . . 14

2.3 The assumptions of the Bree test . . . . . . . . . . . . . . 15

2.4 VrSuite verification . . . . . . . . . . . . . . . 16

3 Thermal-Stress analysis of The Canadian SCWR reference design 23

3.1 Analysis of the reference design . . . . . . . . . . . . . . 23

3.1.1 Thermal analysis ................... 23

3.1.1.1 Domain ................. 24

3.1.1.2 Meshing and the Material properties . . . . . . . 24

3.1.1.3 Boundary condition and Initial condition . . . . . . 25

3.1.1.4 Governing equations ............ . 27 
$3.1 .2 \quad$ Stress analysis . . . . . . . . . . . . . . . . . . . . . 29

3.1.2.1 Displacement and effective stress . . . . . . . . . 29

\section{Optimization Of Temperature Profile Of Startup-Shutdown 34}

4.1 Introduction . . . . . . . . . . . . . . . . . . 34

4.2 Problem definition $\ldots \ldots \ldots \ldots \ldots \ldots \ldots$

4.2 .1 Optimization requirement . . . . . . . . . . . . . 41

4.2.1.1 Design space and design of experiment matrix . . . . 41

4.2.1.2 Objective Function . . . . . . . . . . . . . 43

4.2 .1 .3 Constraints . . . . . . . . . . . . . . . . 43

4.2 .2 Methods of Optimization . . . . . . . . . . . . . . 45

4.2.2.1 Global Optimization . . . . . . . . . . . . 45

$4.2 .2 .2 \quad$ Local Optimization . . . . . . . . . . . . . . 46

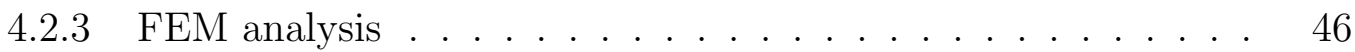

4.2 .3 .1 Domain . . . . . . . . . . . . . . . 47

4.2.3.2 Meshing and the Material properties . . . . . . . 47

4.2.3.3 Boundary condition and Initial condition . . . . . . 48

4.2 .4 Shake down Analysis . . . . . . . . . . . . . . . . . . 48

4.3 Result and discussion . . . . . . . . . . . . . . . . . . . . . . 49

4.4 Which points in the plenum are most susceptible to crack initiation? 52

4.5 Optimized startup scenario $\ldots \ldots \ldots \ldots \ldots \ldots$

4.5 .1 Objective Function $\ldots \ldots \ldots \ldots \ldots \ldots$

4.5.2 The best scenario for ramping up temperature and time $\ldots \quad 55$ 
4.5.3 The effect of pressure ramping on the optimized thermal start

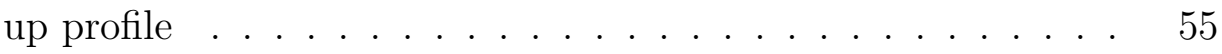

4.6 Shake down analysis for the most susceptible points for initiation of a

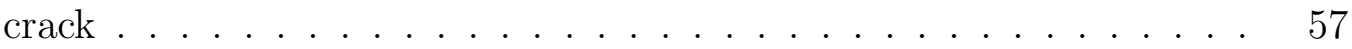

5 Optimization Of GEN IV plenum geometry $\quad 70$

5.1 Introduction . . . . . . . . . . . . . . . . 70

5.2 Problem definition . . . . . . . . . . . . . . . . . . . . 71

5.2.1 Tube sheet. . . . . . . . . . . . . . 72

5.2 .2 Plenum .............................. 72

5.2.3 Nozzle and Vessel mesher . . . . . . . . . . . . . . . 73

5.2.4 Constraints ....................... 74

5.3 The sensitivity of vessel mesher to its parameters . . . . . . . . . 75

5.3.1 Effect of changing nozzle angle and nozzle height on EPS . . . 76

6 Conclusion and possible Future works $\quad 83$

6.1 Bree test verification with VrSuite . . . . . . . . . . . 83

6.2 Reference analysis conclusion . . . . . . . . . . . . . . 84

6.3 Optimization of startup and shut down applied pressure and temper-

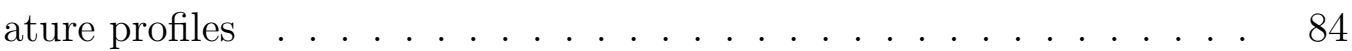

6.4 Parametrization and future work . . . . . . . . . . . . 85

6.4.1 Future works . . . . . . . . . . . . . 86

$\begin{array}{ll}\text { References } & 98\end{array}$ 


\section{List of Tables}

3.1 Summary of the GEN IV insulation based on SCWL [18] . . . . . . . 26

4.1 The comparison of CPU time for doing stress or thermal analysis for different meshes and their number of elements. . . . . . . . . . . . 50

4.2 The difference of EPS and error percentage based on 2D axisymmetric analysis result in effective stress of three mesh cases with 2D axisymmetric mesh. . . . . . . . . . . . . . . . . . . 52

1 The temperature dependent material properties of SA533 that was used by thermal solver. . . . . . . . . . . . . . . .

2 The temperature dependent material properties of SA533 that was

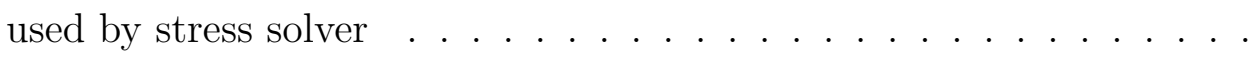




\section{List of Figures}

2.1 Stress Strain curve that shows ratcheting [11] A) With translation of the yield surface from its origin B) Without restriction on kinematic movement of yield surface . . . . . . . . . . . . . . . . . . 8

2.2 Stress Strain curve for uniaxial test that depicts alternating plasticity

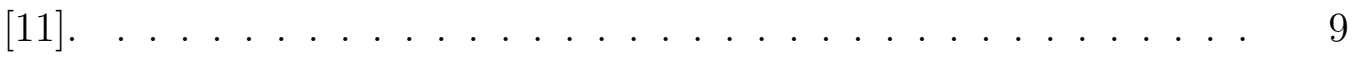

2.3 Three states of plastic strain response to fatigue load [12]. . . . . . . 9

2.4 THe schematic of Bree domain [12] the defines the outer side $(x=$ $1 / 2 d)$, inner side $(x=-1 / 2 d)$, the dash line shows where the mean temperature is constant. . . . . . . . . . . . . . . . . 10

2.5 Modified R5 model that is presented in [15] . . . . . . . . . . 13

2.6 The Bree diagram with six distinct region [7]. Two shake down regions $\left(S_{1}\right.$ and $\left.S_{2}\right)$, two ratcheting regions $\left(R_{2}\right.$ and $\left.R_{1}\right)$, reverse plasticity $(R P)$ and elastic region $(E) \ldots \ldots \ldots \ldots$

2.7 The temperature of the most inner and outer points of cylinder. . . . 18

2.8 The maximum increment of EPS for total time of analysis which suggest shake down of the cylinder. . . . . . . . . . . . . . 
2.9 Nodal stress changes along a line from an inside point of cylinder to an outer point. a) In VrSuite 3D simulation b) In Bree's 1D analytical solution [7]. The ts denotes time step and 6 lines denotes 6 components of stress. . . . . . . . . . . . . . . . .

2.10 Nodal stress changes along a line from an inside point of cylinder to an outer point. a) In VrSuite 3D simulation b) In Bree's 1D analytical solution [7]. The ts denotes time step and 6 lines denotes 6 components of stress. . . . . . . . . . . . . . . . .

2.11 The principal nodal stresses that are visualized as a cube for each Gauss

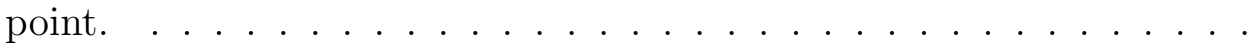

3.1 Schematic view of total reactor mesh; a) isometric view b) cut plane

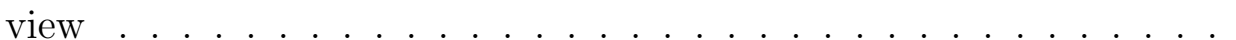

3.2 Schematic view of plenum A) isometric view B) section view of the reactor plenum (bottom tube sheet not shown). . . . . . . . . . .

3.3 Maximum and minimum temperature in the plenum after completing one complete thermal cycle. . . . . . . . . . . . . . . .

3.4 Maximum displacement of plenum A) Deformed geometry B) Undeformed geometry ...................... . . . . 32

3.5 Maximum effective stress A) Cut plane view B) Top view. . . . . . . . 32

3.6 Maximum EPS A) Isometric view after 10 cycle. B) Section view of the reactor plenum after 1 cycle. . . . . . . . . . . . . . . . 33

3.7 EPS for three points after 10 cycles. . . . . . . . . . . . . . 33

4.1 Constant pressure startup system for HPLWR. [22]. . . . . . . . . . 37 
4.2 Constant pressure startup curve for SCLWR-H. [23] . . . . . . . . . . 37

4.3 Sliding pressure startup curve for HPLWR. [22] . . . . . . . . . . . . 38

4.4 Start up stages for FR with bypass system [21] . . . . . . . . . . 38

4.5 By pass system in FR which feeds the main reactor and the path of water to turbines $[21] \ldots \ldots \ldots \ldots$

4.6 The mesh that was employed in the $2 \mathrm{D}$ axisymmetric mesh. . . . . .

4.7 EPS is shown as a contour plot. a) 3D axisymmetric mesh with 40 circumferential elements. b) 3D axisymmetric mesh with 120 circumferential elements. . . . . . . . . . . . . . . . . . .

4.8 Showing the maximum EPS in the domain. Three points that have the maximum EPS are shown. . . . . . . . . . . . . . .

4.9 Design space for DOE temperature. Each profile is associated with one point in the design space $\left(t_{i}, T_{i}\right) \ldots \ldots \ldots \ldots$

4.10 Pressure as a function of temperature with a cubic function fitted to it. The gray line is the one with temperature shifted 20 degrees to the

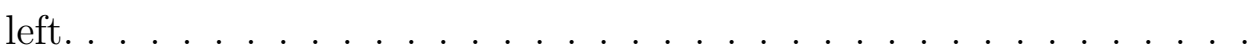

4.11 Arbitrary cases which shows; a) The ambient temperature applied to the inner surface of plenum. b) The corresponding pressure curve. . .

4.12 Optimized value of DOE temperature. a) Optimized value of DOE temperature and its DOE time with the objective function value. b) evolution of objective function during Kriging iterations. . . . . . .

$4.132 \mathrm{D}$ plot of objective function EPS as function of DOE temperature, $T_{2}$ and DOE time, $t_{2}$ for the Sutanto start up scenario. . . . . . . . 
4.14 Optimized value of DOE temperature. a) Optimized value of DOE temperature and its time with the objective function value. b) evolution of objective function during Kriging iterations. . . . . . . . . .

$4.152 \mathrm{D}$ plot of objective function EPS as function of DOE temperature, $T_{2}$ and DOE time, $t_{2}$ for the case which the pressure is ramped up linearly

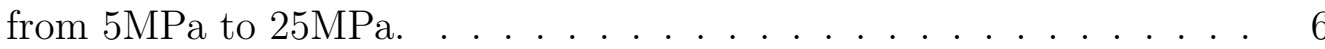

4.16 Optimized value of DOE temperature. a) Optimized value of DOE temperature and its DOE time with the objective function value. b) evolution of objective function during Kriging iterations. . . . . . .

$4.172 \mathrm{D}$ plot of objective function EPS as function of DOE temperature, $T_{2}$ and DOE time, $t_{2}$ for the case which the pressure is ramped up linearly from $10 \mathrm{KPa}$ to $25 \mathrm{MPa} . \ldots \ldots$. . . . . . . . . . . . 66

4.18 Pressure cycles used to find shake down bounds. . . . . . . . . . . . . 67

4.19 The upper and lower bounds of shakedown with comparing of the EPS for each loading case that is shown in Fig. 4.18 for point1. . . . . . . 67

4.20 The upper and lower bounds of shakedown with comparison of the EPS for points 2 and $3 . \ldots . \ldots . \ldots . \ldots . . \ldots 68$

4.21 Displacement versus time for point 1 in the domain which is shown to be increasing. . . . . . . . . . . . . . . . . .

5.1 Parametrized tube sheet mesher; a) 2D view b) Sub-domains of tube sheet. ........................... 73

5.2 Parametrized Plenum. . . . . . . . . . . . . . . . 78

5.3 parametric FEM mesh generated with 3 separate parts. . . . . . . . 78 
5.4 The meshed vessel with vessel that is most similar to the mesher to the AECL design . . . . . . . . . . . . . . . . . . . . 79

5.5 The maximum EPS for vessel that is similar to AECL geometry that is meshed with vessel mesher. . . . . . . . . . . . . . . . . 80

5.6 The maximum EPS as a function of the angle between nozzles. . . . . 81

5.7 The maximum EPS change as a function of the height of nozzles. . . 82

1 Contour Plots of Temperature Field for Semi infinite Bar. . . . . . . . 93

2 Comparison of FEM solution and exact solution for 3 different times. $\quad 95$

3 The error which made by choosing wrong time steps or element length. 96

4 Comparison of FEM solution with end effect and eliminating end effect. 96

5 Comparison of temperature field for 2D, 3D and exact solution. . . . 97

6 Comparison of nodal stresses for 2D, 3D and exact solution at the last time step. . . . . . . . . . . . . . . . . . . . . . . 97 


\section{Chapter 1}

\section{Introduction}

\subsection{Background}

Motivated by the loss of life and property damage due to boiler explosions in previous years, the first version of the ASME Pressure Vessel and Piping Code was published in the 1911. Since then, the ASME Boiler and Pressure Vessel Code (B \& PV) [1] has been developed. The ASME B \& PV codes provides the requirements for designing pressurized equipment by specifying simplified design rules based on current best practice to minimize the risk of failure. Such a method is called "design by rule". As the designed structures became more complicated and sophisticated numerical tools were developed, the ASME B \& PV has been developed to cover design cases that used analysis results. This method is called "design by analysis". If the designer can convince the ASME committee through detailed analysis, the design would be accepted under the code.

To prove the validity of the design, the value of one or more specified objective 
function must be computed. The objective function could change as new knowledge is acquired [2]. This computation is made by contributions from parameters that are called design variables. The design variables should have constraints that bound the range of each design variable. A set of these constraints defines a design space. Constraints define the upper bound and lower bound of each design variable. There should be minimum increment size of changing a variable within the constraint range. See [3].

To begin design of experiment procedure, designer should create a prototype which is reasonable and be applicable to run the the experiments [2]. This is called the reference design. All variables do not have the same effect on the objective function. The sensitivity of the objective function to each parameter could be measured using Taguchi design of experiment (DOE) matrices or Box-Behnken design of experiment (DOE) matrices. G. Box is one of the pioneers in doing optimization by design of experiment method [2]. Box used the design of a paper helicopter to show the concept of design of experiment optimization. The objective function which he used was maximizing the flight time of a paper helicopter. Recently, Asadi et al. [4] and [5] tried to use optimization based on DOE to minimize the deformation in a welded pipe and Masabuchi bar [6].

As a part of worldwide activities directed to the development of the next generation nuclear reactors, Atomic Energy of Canada Ltd (AECL) developed a reactor core concept that uses supercritical water as coolant. This concept is called Canadian super critical water reactor (SCWR). Although, the designed core was based on ASME rules, there was a need to analyze the thermo-structural behavior of the core and optimize the design if necessary. 
This work is an attempt to develop and demonstrate a new methodology for the design optimization of pressure vessels such as the inlet plenum of Canadian SCWR reactor core. This methodology is based on a thermo-viscoelastic-plastic model of the Canadian SCWR reactor core, that produces reasonably accurate and time- and cost-efficient solutions. This analysis approach includes a stress analysis coupled with a temperature field and optimization of the 3D transient nonlinear coupled holistic macroscopic model of the plenum. The thermal stress and the energy equation are coupled in the sense that for each time step the displacement FEM solver gets transient temperatures from the heat equation. For each time step, the heat equation gets displacements from the displacement stress solver. The time steps need not be synchronized. The heating effect of the elastic pressure time volume change is ignored and the heating effect of the stress times the plastic strain rate is ignored. Both are negligible in the applications considered in this thesis. The model is also used for the preliminary optimization of the design of the reactor core plenum.

\subsection{Scope and Organization of the Thesis}

The main purpose of this thesis is to demonstrate a robust and efficient methodology for the optimization of the Canadian SCWR reactor core.

Chapter 2 deals with the verification of the FEM software used to model the Bree test. The Bree test is an important reference for doing shake down analysis. The 1D model of the Bree test is simulated with a 3D cylinder in VrSuite. The compatibility of results will be shown.

Chapter 3 introduces the reference design. The geometry of the reference design 
is provided by AECL in the form of IGES files. A coupled thermal/stress analysis with ramping up pressure and temperature design for two days, has been done. The chapter details the methodology used for the analysis, mentions the assumptions and simplifications, elaborates the points that are susceptible to initiate a crack, states essential analysis parameters, such as mesh parameters, BCs and governing equations, and provides an interpretation of the obtained results. It also mentions the possibility of shake down of different points in the plenum by running ten similar startup-shut down cycles.

Chapter 4 describes the optimization of ramped temperature profile with pressure that is a function of saturated water temperature. The objective function for optimization is to minimize the effective plastic strain (EPS). Also the difference in computation time for stress and thermal analysis between 2D, 3D axisymmetric mesh and 3D non axisymmetric mesh was discussed. The effect of changing pressure on the optimized value was investigated. Finally, the shake down analysis is done on the 2D axisymmetric mesh to find the maximum pressure that leads to shake down for different points in the plenum.

Chapter 5 describes the parametrization of the plenum design. In this chapter, the vessel mesher (as well as three other parametric meshers) as a robust parametric mesh of the plenum was introduced. Also the constraints to preserve the topology of the mesh was discussed. The sensitivity of the objective function to design variables was considered. However, the lack of some sensitivity algorithm such as Taghuchi was noted.

Chapter 6 summarizes of the work done with respect to the main goal of the work, which is the development of a methodology for the design and optimization 
of the Canadian SCWR reactor core, and with respect to the thermal-mechanical analysis of the plenum, giving the overall conclusions about the effectiveness of the developed methodology and proposing possible future research activities and ways for improvement of the presented numerical model, and the existing Canadian SCWR mechanical design. 


\section{Chapter 2}

\section{Verification of Bree test with}

\section{VrSuite}

\subsection{Introduction}

The Bree test was originally developed to model the behavior of the fuel sheath of a fast breeder reactor under cyclic load conditions [7]. It has been used by many researchers as a benchmark test for components failure modes under cyclic loading. Any structure can fail by imposed mechanical or thermal cycling loads. It depends on the state of strain versus time curve. Three conditions that may happen are:

- If the total strain tensor component in each loading cycle decreases and in the limit of infinite cycles, the rate of change of the plastic strain approaches zero the material is said to shake down. (It could fail by low-cycle fatigue first). In this condition, doing more cycles on the structure would not produce any additional plastic deformation (total strain tensor components remain constant). Rupture 
in this state will be governed by high cycle fatigue which will be in the elastic region.

- If the total strain tensor component in each loading cycle increases with constant amplitude and the total displacement increases simultaneously, the material is said to ratchet. In this case, some components of the total plastic strain in each cycle will increase with constant amplitude and this increase will lead to the final rupture of material. See the Fig. 2.1 and 2.3 [8].

- If a plastic strain tensor component oscillates with the same amplitude for all loading cycles and the maximum plastic strain tensor components remain bounded by a constant value in each cycle, the material is said to be in alternating plasticity. The rate of change of the maximum and minimum strain tensor components during each cycle will be zero. This state is called cyclic plasticity. Rupture in this state will be governed by low cycle fatigue. See the Fig. 2.2 and $2.3[9]$ and [10].

Figure 2.3 depicts three types of plastic strain response to cyclic loads. For the red line, the total maximum strain remains constant while the strain tensor component is fluctuating. This is reverse plasticity. The green line illustrates ratcheting, where the total maximum strain and minimum strain tensor components increase. The blue line represents shake down where, the amplitude of fluctuation of the plastic strain tensor components converge to zero and the maximum total strain equals the mean value of fluctuating strain tensor component value.

To determine the behavior of material undergoing cyclic loading, many papers have been published in the past five decades. One of the first papers was published 


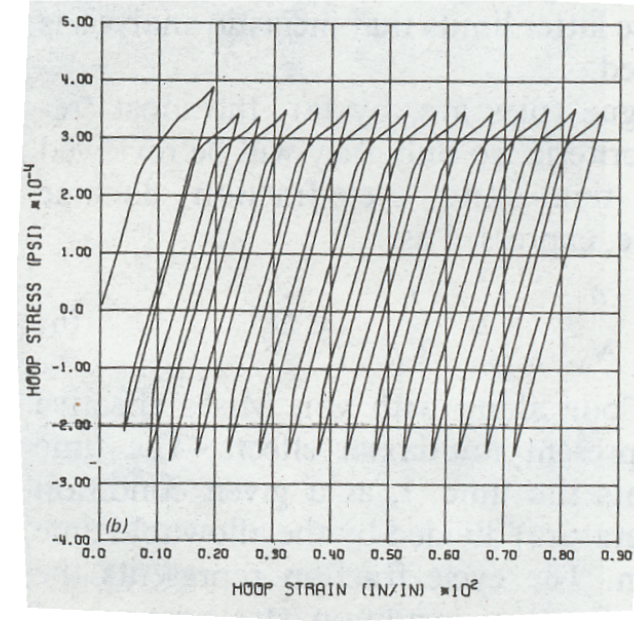

A)

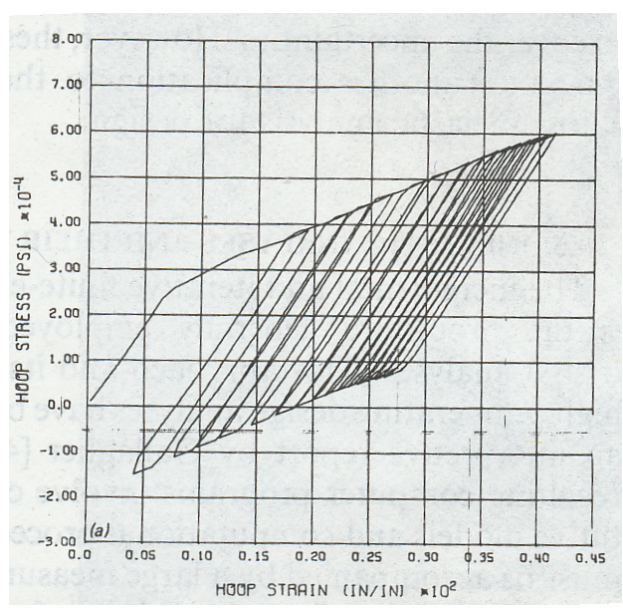

B)

Figure 2.1: Stress Strain curve that shows ratcheting [11] A) With translation of the yield surface from its origin B) Without restriction on kinematic movement of yield surface

by J. Bree [7] in 1967. Bree developed a 1D model for a fuel sheath in a fast breeder reactor. The fuel sheath cylinder was pressurized by the gas from fusion which was accompanied by a heat flux. Mechanical and thermal loading was applied to the fuel sheath. In Bree's model, the material has constant elastic modulus which was temperature independent. The numerical method assumed a non-work hardening material. Bree extended the definition of the problem to work hardening material in [13]. Bree tried to model a 1D strain problem. To achieve such a 1D model, the following simplifications were assumed:

1. The height of the cylinder is much larger than its thickness.

2. The wall thickness of cylinder is much more smaller than its radius. 


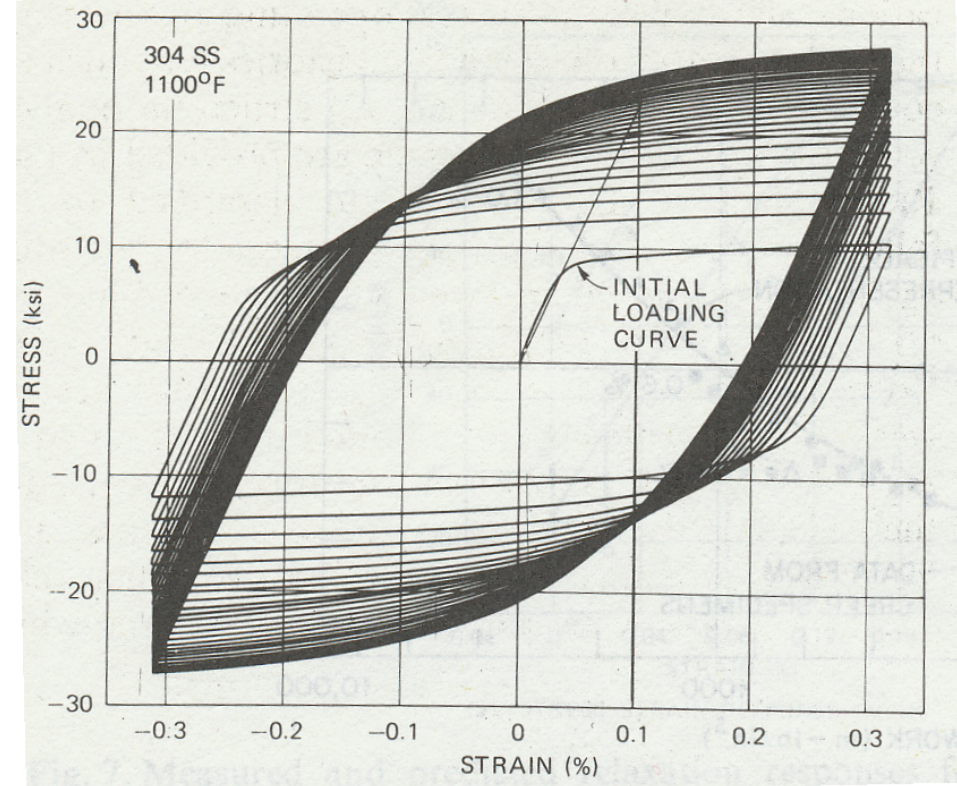

Figure 2.2: Stress Strain curve for uniaxial test that depicts alternating plasticity [11].

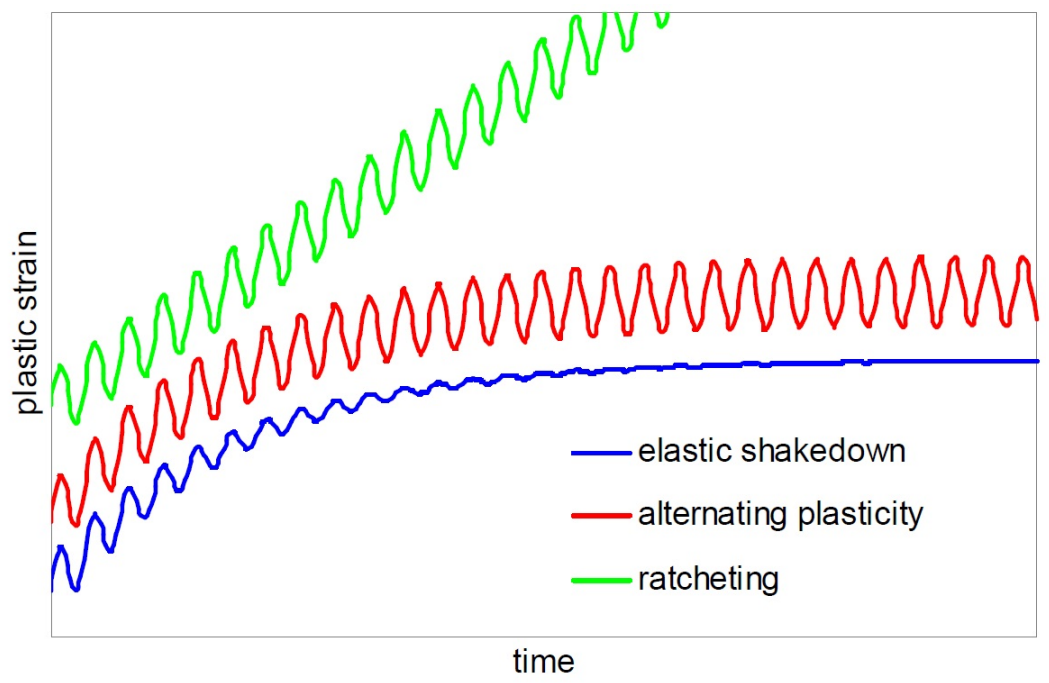

Figure 2.3: Three states of plastic strain response to fatigue load [12]. 
The hoop and axial strains depend on the coordinates of elements and the radial stress could be neglected. The model should be constrained in such a way that the domain doesn't bend in the hoop and axial directions. The best way to explain the Bree problem is to use an axisymmetric cylinder. The hoop stress has a linear change from the mid surface. The mean axial stress should be zero, which means the cylinder ids free to expand in the axial direction. The temperature changes linearly (assigned temperature to each node) in the radial direction in the cylinder wall. The mid wall temperature should be always constant (See Fig. 2.4). If the temperature in the right hand side (outer) of mid plate is increasing the left hand side (inner) is decreasing with same rate of change as the right hand side (outer) and vice versa. This temperature variation will cause compression in the left side (inner) and tension in right side (outer) of plane in start up and the opposite in shut down.

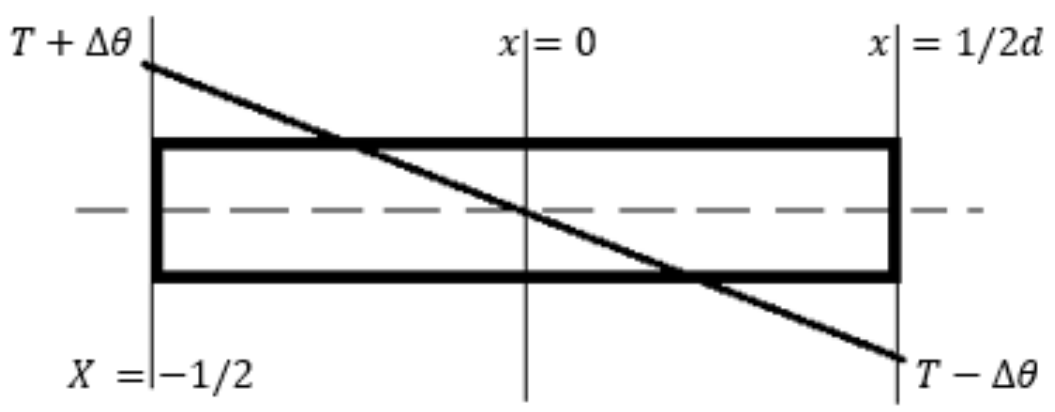

Figure 2.4: THe schematic of Bree domain [12] the defines the outer side $(x=1 / 2 d)$, inner side $(x=-1 / 2 d)$, the dash line shows where the mean temperature is constant.

By plotting the $\sigma_{t}$ (thermal stress) versus $\sigma_{p}$ (pressure), Bree identified six distinct regions which are: elastic region, two shake down regions, cyclic plasticity region and 
two ratcheting regions. By attaching the reverse plasticity line and shake down line the boundary between ratcheting (or cyclic plasticity) and shake down regions will be well defined. So tracking the ratcheting (onset of ratcheting) line is the most critical point of doing a Bree analysis. The behavior of the cylinder in the first cycle of start up-shut down cycle in ratcheting region, $R_{1}$, is expressed as: The stress increases during start up to the plastic region. Then the stress in both the first half of the cycle (in half of the wall) and the second half of the cycle remain constant, where as, the hoop strain increases linearly in start up. During shut down the rate of strain change is negative. In the next start up stress increases linearly from inside to the other side. They also claim that the ratcheting area $R_{2}$ can be accessed when there is a axial or hoop stress from internal pressure.

They also created a Bree diagram for the case with creep too. Creep strain is irreversible and it's time dependent. The stress is not uniform in the presence of creep. For this case, the ratcheting region, $R_{1}$, is described as follows: The stress increases linearly during start-up, the plastic strain remains constant. However, the creep strain increases in the whole wall in normal operation, the stress relaxes and the strain remains constant. Due to the decreasing temperature in shut down the creep strain rate goes to zero and the creep strain remains constant. In a general case the summation of thermal stress $\left(\sigma_{t}\right)$ and pressure stress $\left(\sigma_{p}\right)$ should be less than the yield stress $\left(\sigma_{y}\right)$. In the case in which creep plays a role, the pressure stress $\left(\sigma_{p}\right)$ is multiplied by creep coefficient $(\lambda)$. The $\lambda$ is temperature dependent and varies from 0.7 (when $\sigma_{t}=\sigma_{y}$ ) to 1 (when $\sigma_{t}=0$ ). It's clear that by substituting this inequality instead instead of the inequality for case one, the elastic line will shift to a horizontal line which will result in shrinkage of elastic region. 
Note that by considering the effect of irradiation on the materials, the yield stress increases and the ductility of the metal decreases.

The need for a comprehensive model which explains the procedure of doing analysis on the structure that imposed thermal and mechanical loads, always exists. The R5 model [14] is one of the models which are used widely for life assessment. Chen et al. [15] presented a simplified $\mathrm{R} 5$ model. The $\mathrm{R} 5$ flowchart for a structure that is subjected to thermal and mechanical loads is shown in Figure 2.5. The flow chart is divided into two separate parts: Part I discusses shakedown and limit analyses, creep rupture analysis and the evolution of creep during cyclic loading without residual stresses. Part II considers the residual stress field in the analysis. Part II of the flowchart is discussed in detail in [16]. Chen claims there are two inherent disadvantages with R5 models: The range of material data does not support the constitutive description of the problem and in a full inelastic problem, the level of loading which determines the significant behavior of the material is not fully described. A new method, linear matching method (LMM), was developed in the last few years [17][10]. The LMM tries to apply a non linear material to a linear material problem. It also tries to present a powerful model that could capture the upper bound of shake down [13].

More important than finding the upper bound and lower bound of shake down is the application of the shake down theorem in designing a structure that tolerates the fatigue load. One of the papers that discusses the design of refinery reactors based on the shake down theorem, is presented by the Fluor company [13]. They tried to provide some guidelines for testing new ASME Section VIII, Division 2 Code as an example of User's Design Specification for hydro cracking reactors. Instead 


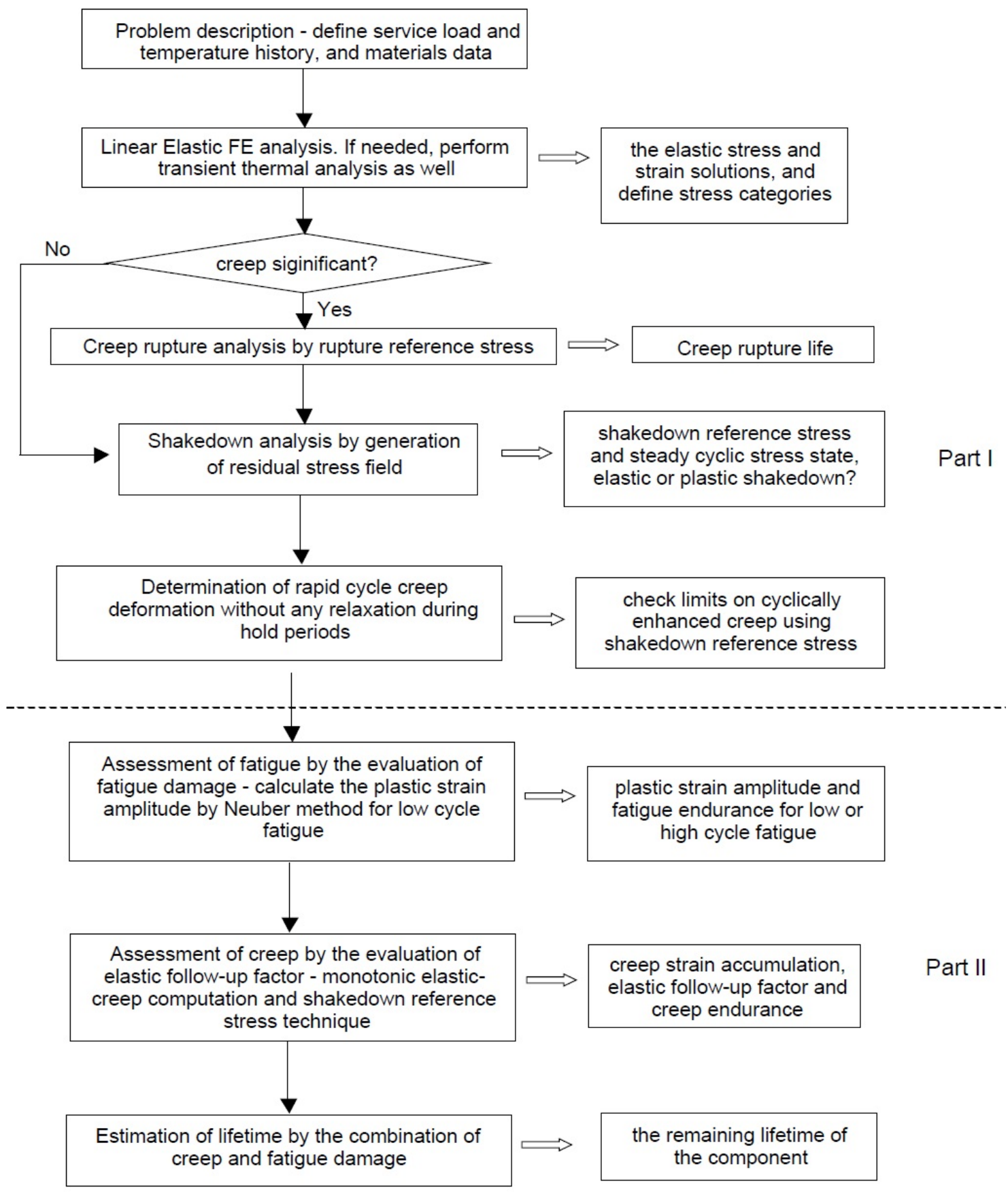

Figure 2.5: Modified R5 model that is presented in [15]. 
of analyzing the whole design space, five critical points have been chosen. They analyzed the creep and fatigue tests for critical components. They studied these pressure/temperature cycles:

1. Normal Operating Condition. 2. Initial / Normal Start-up. 3. Normal (Planned) Shutdown. 4. Unplanned/Emergency Shutdown. 5. Start-up After Unplanned Shutdown.

They used a simplified, approximate ratcheting analysis to demonstrate elastic shakedown. After passing two complete normal startup and shut down cycles, they plotted the stress strain hysteresis (with an elastic perfectly plastic model) and total strain versus time plots. Because both plots suggest shake down after two cycles, they claim the structure will shake down and the controlling mechanism for determining the life of the reactor will be defined by creep. The total strain will be the sum of creep strain and strain from heating and pressure load. By using the total strain, the number of startup shut down cycles that the structure is allowed to have will be evaluated.

\subsection{Definition of Bree problem}

As was discussed in the first chapter, the Bree cylinder problem has been studied by a number of researchers (Bree, 1967) [7]. Bree analyzed the elasticplastic behavior of a thin cylindrical tube subjected to internal pressure and cyclic high temperature gradient across the tube thickness. Bree (1967) [7] used a simple one-dimensional model and assumed a linear temperature drop distribution across the cylinder thickness. The Bree test was developed to model cyclic loading of the sheath of a fast 
nuclear reactor fuel element. To have a better understanding of the six regions that Bree identified for thermal versus mechanical loading (described in first part of this chapter), see the Fig 2.6.

\subsection{The assumptions of the Bree test}

Bree made the following assumptions to devise a one dimensional model:

1. In the first paper of Bree (1967) [7], the measured strain was hoop strain.

2. The Tresca yield criteria was used.

3. The Poisson's ratio was set to zero $(\nu=0)$.

4. Bree assumed rate independent plasticity, i.e., viscosity is zero $(\mu=0)$.

5. The material was elastic - ideal plastic, i.e., zero strain hardening modulus $(\alpha=0)$.

6. When the analysis included creep, Bree assumed creep completely relaxes the internal stress during a power on stage.

7. Bree parameterizes temperature by a mean temperature and a temperature gradient. In other words, the mid-surface was assigned to the mean temperature and the temperature gradient generates temperatures above and below the mean temperature.

8. In the last Bree paper the axial loading was added to the cylinder. 


\section{$2.4 \quad$ VrSuite verification}

To verify the analysis of the Bree test with VrSuite, a new material was defined $\left(\sigma_{y}=208 \mathrm{MPa}, \kappa=24.6\left[\mathrm{~W} / \mathrm{m}^{\circ} \mathrm{K}\right], \mu=0, \nu=0, E=0.186 e 12 \mathrm{~Pa}\right.$ and $\left.\alpha=0\right)$.

To approximate a thin cylinder for the Bree test the ratio of inside radius to the thickness $(R / t)$ was set to forty. The rectangular plate (in the $x y$ plane) mesh that has two element in thickness and eight element in radius was rotated wrt the $z$ axis to generate 64 circumferential elements. The $x z$ and $y z$ planes are restricted in the $\mathrm{y}$ and $\mathrm{x}$ direction respectively and the mid plane of the cross section is fixed in the $z$ direction.

A cyclic pressure was applied on the inside surface of the cylinder. The temperature was assigned to each node in the domain. In fact, the temperature varied linearly between inside and out side of cylinder. It means the temperature was assigned as data and was not solved. The middle plane of the cylinder was kept at a mean temperature $\left(300^{\circ} \mathrm{K}\right)$. Where the outer side temperature was $300+\Delta T$ and inside surface temperature was 300- $\Delta T$ and vice versa. Fig. 2.7 depicts the the inner and outer wall temperatures for several thermal cycles as functions of time.

To find the elastic upper bound as a function of the temperature and the pres-

sure. The temperature and the pressure were increased separately until the material started to deform plastically. The maximum pressure and temperature gradient at which the domain begins to deform plastically (elastic bounds) is $4 M p a$ and $90^{\circ} \mathrm{K} / \mathrm{m}$ respectively.

The maximum increment of EPS is shown in Fig 2.8. The increment of EPS decreases exponentially, that suggests shake down of the structure. 
Fig 2.11 illustrates each Gauss point that is visualized with a rectangular blocks. The colors of the six faces of the cube show the components of principal stresses at time step 10. It's obvious that the hoop stress is the component that has the highest value.

To verify that the behavior of S1 region in the Bree diagram could be captured, one point in the $\mathrm{S} 1$ region was chosen. By comparing the principal nodal stresses, it was found that the shake down behavior was captured. Figures 2.9 and 2.10 demonstrate the comparison of nodal stresses in the Bree paper [7] and in VrSuite along the line that starts from the inside point in the cylinder to the most outside one. 


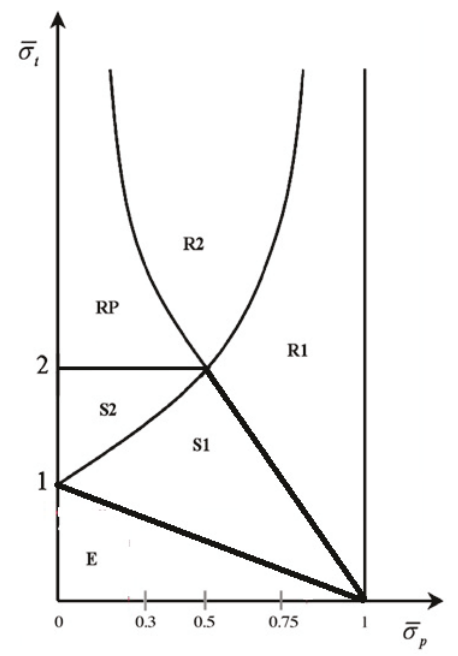

Figure 2.6: The Bree diagram with six distinct region [7]. Two shake down regions $\left(S_{1}\right.$ and $\left.S_{2}\right)$, two ratcheting regions $\left(R_{2}\right.$ and $\left.R_{1}\right)$, reverse plasticity $(R P)$ and elastic region $(E)$.

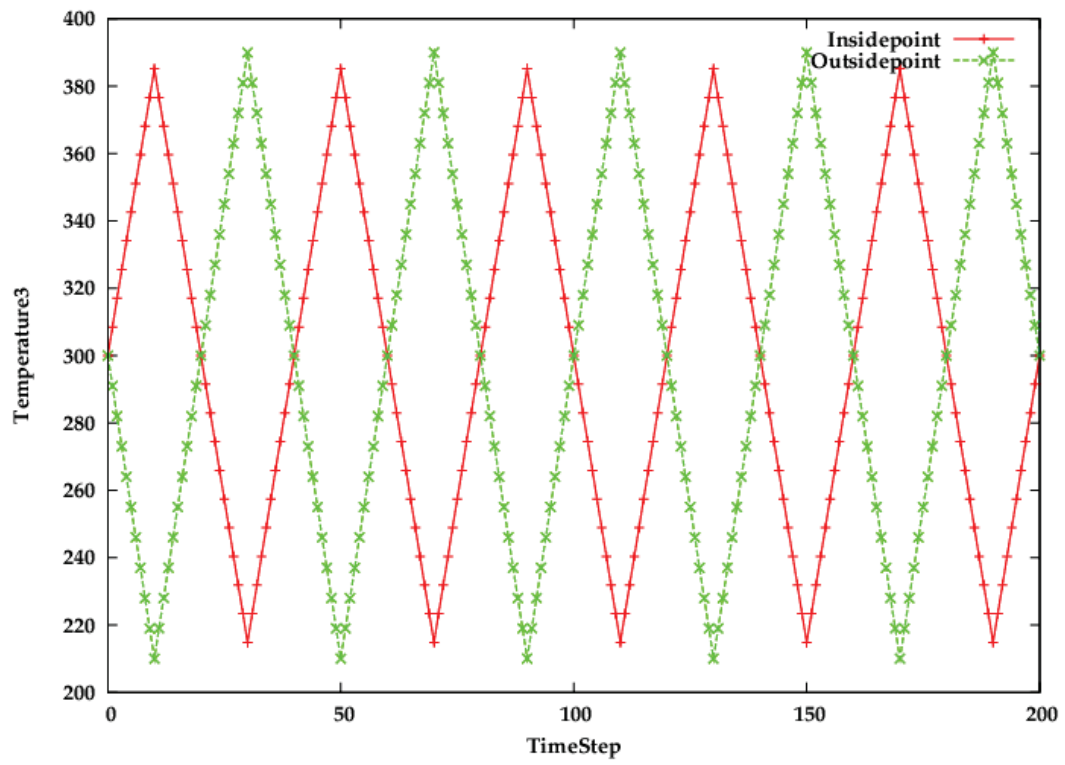

Figure 2.7: The temperature of the most inner and outer points of cylinder. 


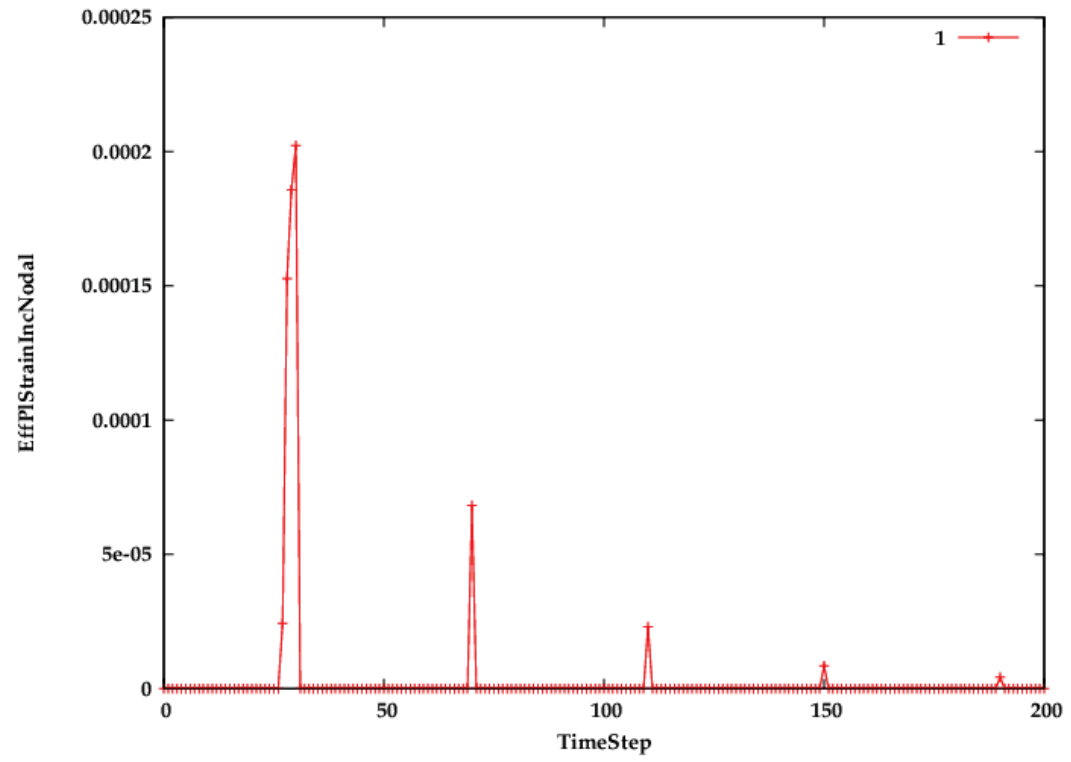

Figure 2.8: The maximum increment of EPS for total time of analysis which suggest shake down of the cylinder. 

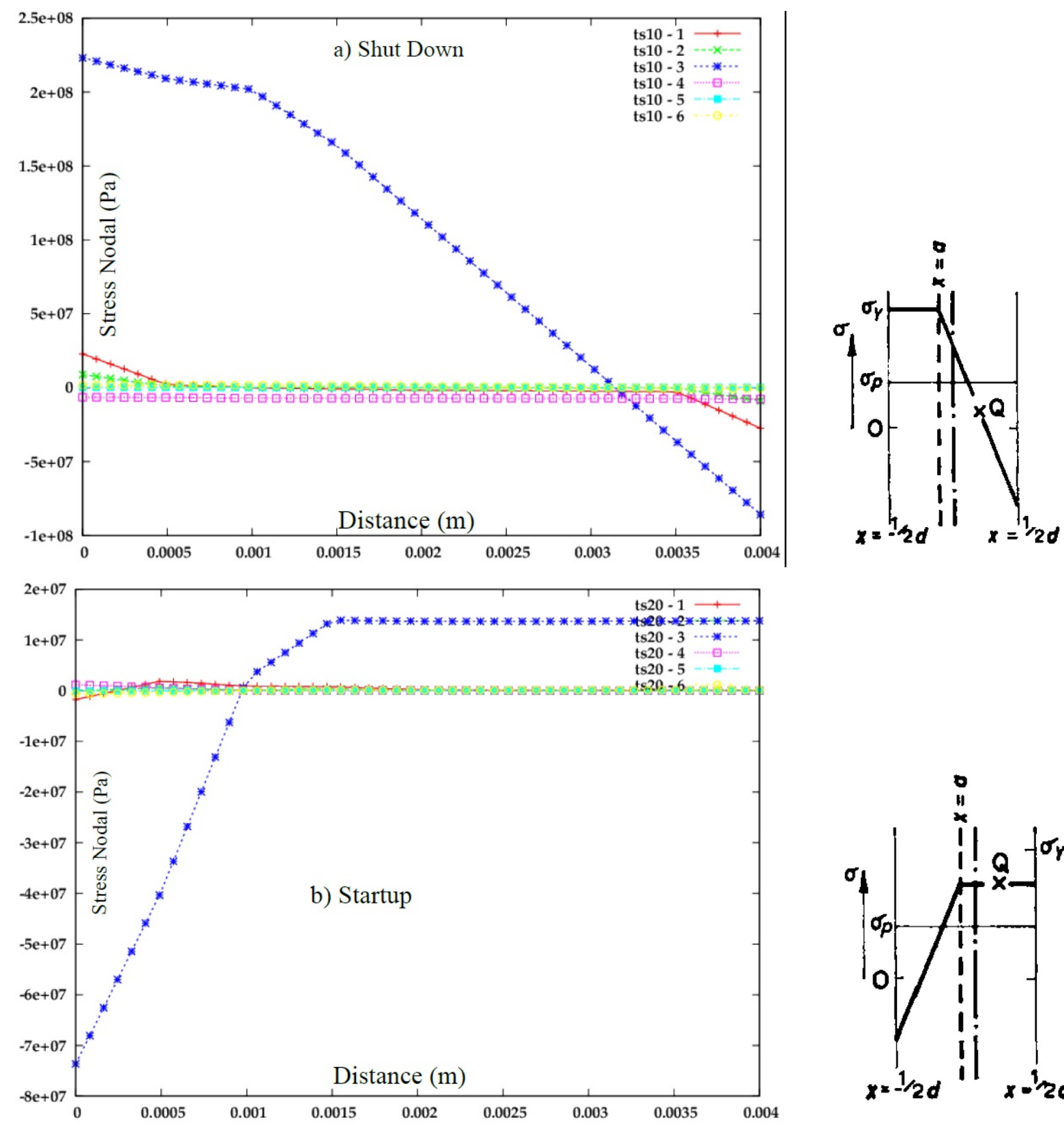

a)

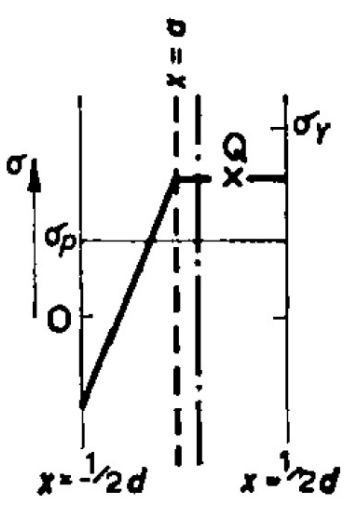

b)

Figure 2.9: Nodal stress changes along a line from an inside point of cylinder to an outer point. a) In VrSuite 3D simulation b) In Bree's 1D analytical solution [7]. The ts denotes time step and 6 lines denotes 6 components of stress. 

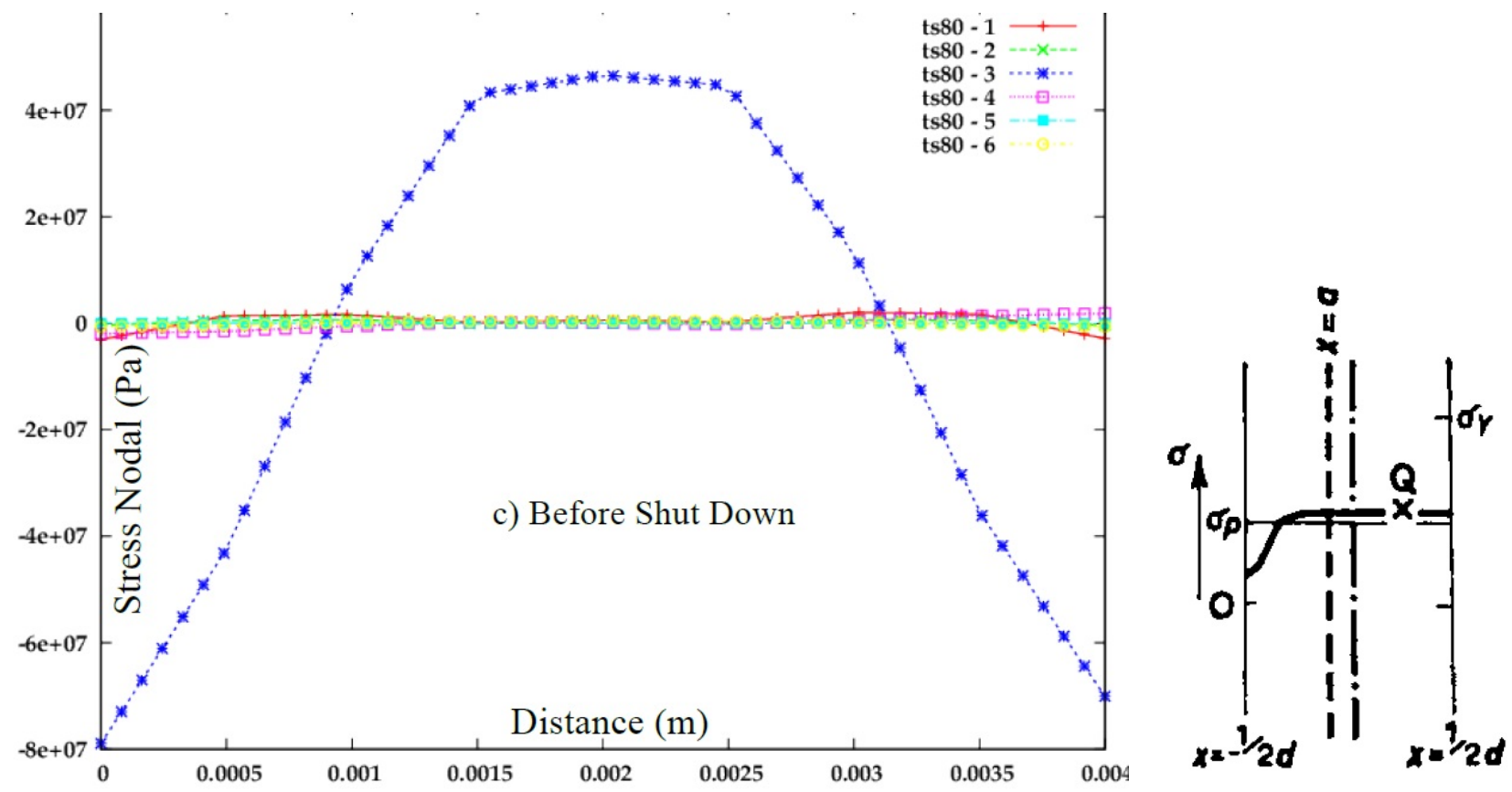

a)

b)

Figure 2.10: Nodal stress changes along a line from an inside point of cylinder to an outer point. a) In VrSuite 3D simulation b) In Bree's 1D analytical solution [7]. The ts denotes time step and 6 lines denotes 6 components of stress. 


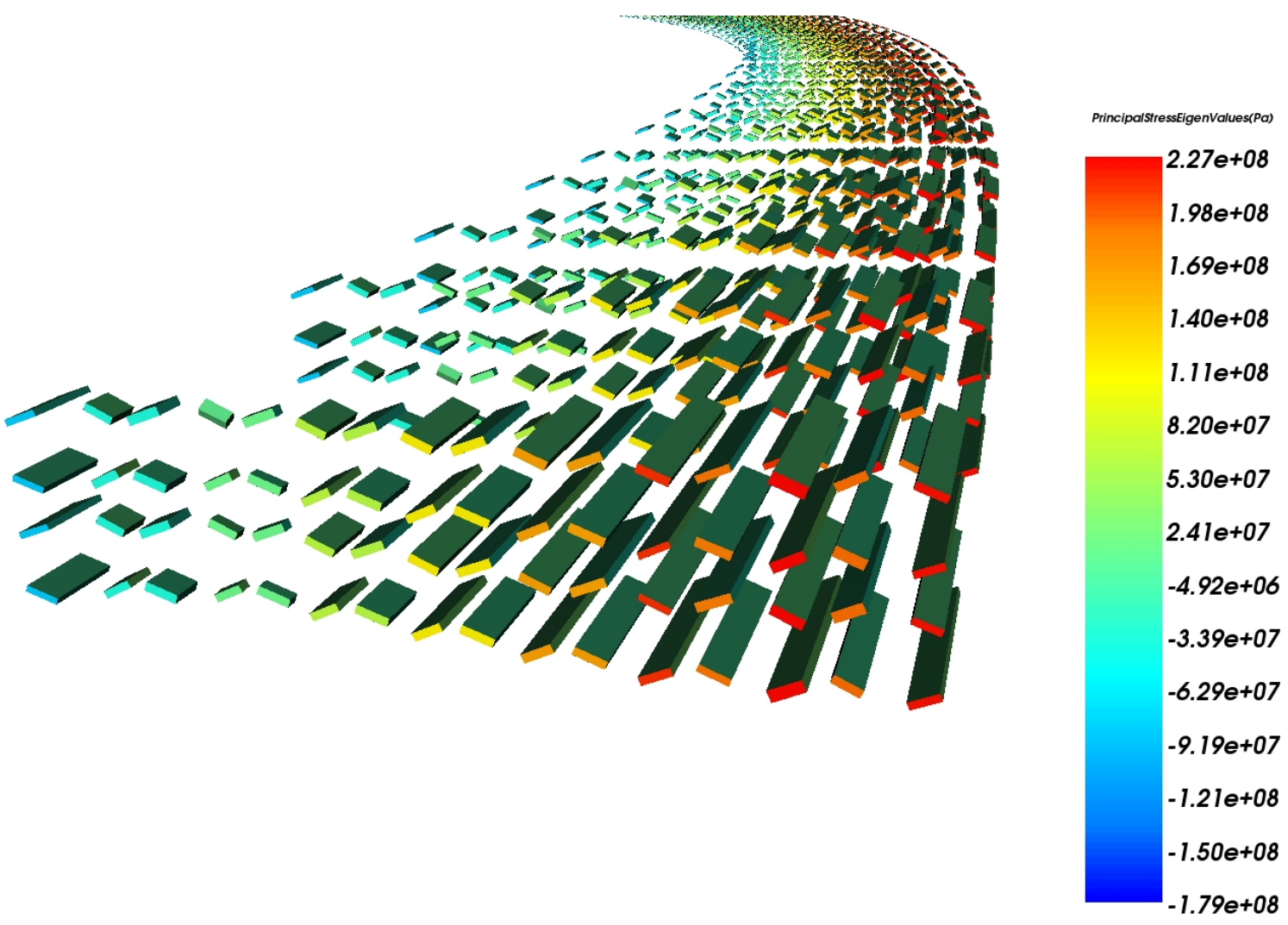

Figure 2.11: The principal nodal stresses that are visualized as a cube for each Gauss point. 


\section{Chapter 3}

\section{Thermal-Stress analysis of The Canadian SCWR reference design}

\subsection{Analysis of the reference design}

A thermal and stress analysis of the design that was provided by AECL was done. Although the immediate goal of this analysis is to verify that the stress levels in the the plenum stay within an acceptable range, the longer term goal of these analyses is to gain a deeper insight into the behavior of the design

\subsubsection{Thermal analysis}

The thermal analysis was performed and transient temperature fields were imported to the stress solver. The details are as follows: 


\subsubsection{Domain}

The initial geometry of the Canadian SCWR was received from AECL as IGES files. This geometry was partitioned into different parts (See Fig. 3.1 for all Canadian SCWR meshed parts). Among all parts, the inlet plenum is the largest pressure carrying component in the reactor core. The plenum itself contains two separated parts: the lid and the body, which are connected to each other by 18 bolt and nuts

joints. The body itself consists of 8 nozzles; 4 smaller cold water inlet nozzles and four larger hot water outlet nozzles which are located below the four inlet nozzles. The clamps which hold the outer header and the support which is holding reactor cores (main constraint). See Fig. 3.2.

The plenum is a part of GEN IV reactor which is contained in a bounding box of approximately $7 \times 7 \times 6.3 \mathrm{~m}$ with the maximum thickness of $92 \mathrm{~cm}$. It weights approximately 600 tons. For this analysis, the holes are omitted from the tube sheet to avoid the need for reactions to balance the force that would be applied by the pressure tubes.

\subsubsection{Meshing and the Material properties}

The plenum is meshed with 8-node hexahedron elements with three conforming mesh parts. Mesh independent analysis is done that means the mesh was refined up to level that the results does not changes with changing of mesh grid size. To resolve stress concentrations, corners are meshed with a fine mesh. The plenum material is SA533 steel. The thermal and mechanical properties of SA533 are listed in Appendix A. 


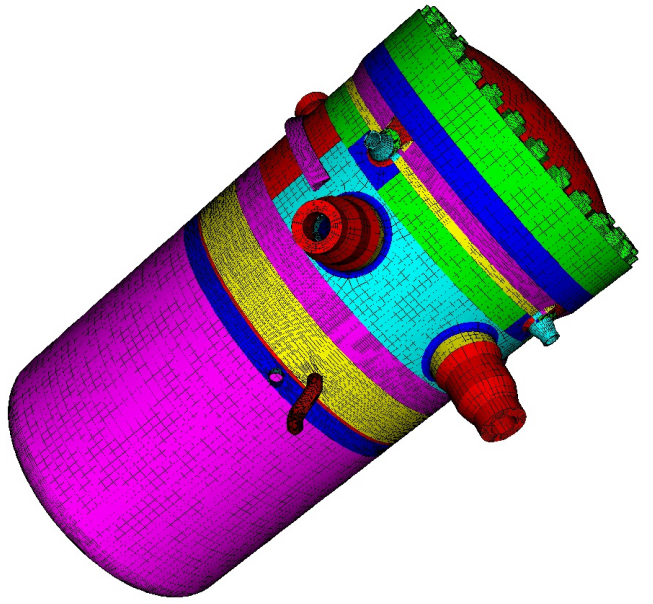

a)

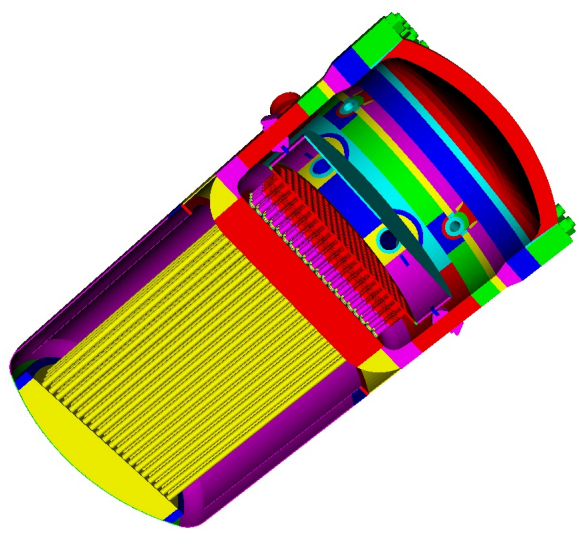

b)

Figure 3.1: Schematic view of total reactor mesh; a) isometric view b) cut plane view

\subsubsection{Boundary condition and Initial condition}

The thermal boundary condition is a convection boundary condition. The convection coefficient for the inside surface is considered to be $1000 \mathrm{~W} / \mathrm{m}^{2 \circ} \mathrm{K}$. Appendix B shows the Churchill and Chu equation for computing the convection coefficient. The outside surface is insulated. Table 3.1 shows the characteristics of the insulation based on the SCWL [18]. The heat transfer coefficient based on the insulator thermal resistance can be computed as follows:

$$
h_{\text {wall }}=\frac{2 k}{d_{\text {in }} \ln \left(d_{\text {out }} / d_{\text {in }}\right)}
$$

$k$ is the insulator thermal conductivity, $d_{i n}$ and $d_{\text {out }}$ are the inner and outer diameters of the plenum, respectively. $h$ is computed convection coefficient. Also the overall equivalent convection coefficient for the different plenum sections are: 


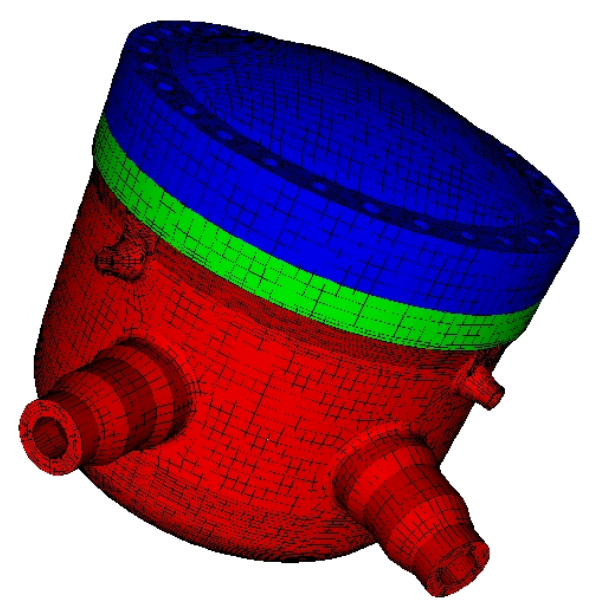

A)

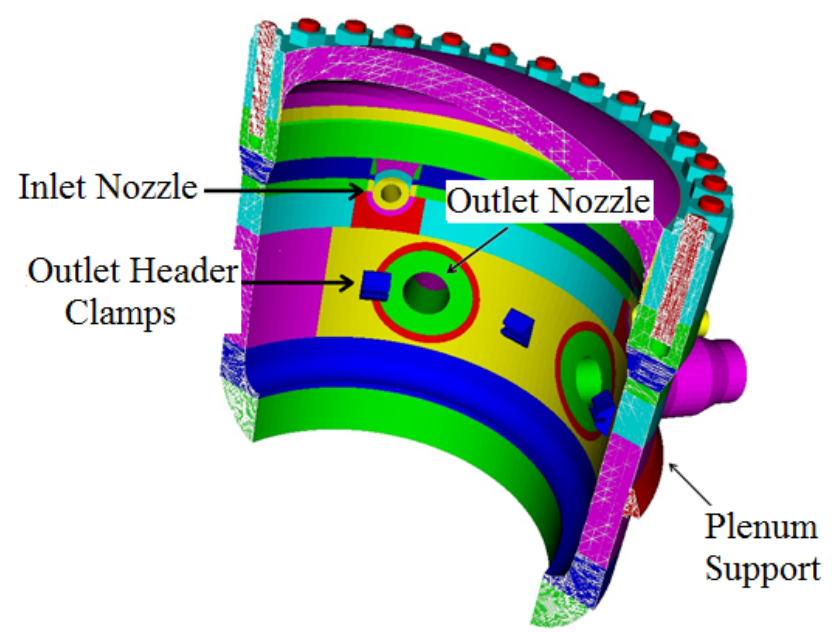

B)

Figure 3.2: Schematic view of plenum A) isometric view B) section view of the reactor plenum (bottom tube sheet not shown).

\begin{tabular}{|l|l|l|l|}
\hline Insulation material & $\begin{array}{l}\text { Insulation } \\
\text { thickness, }[m]\end{array}$ & $\begin{array}{l}\text { Insulator surface } \\
\text { temperature, }\left[{ }^{\circ} \mathrm{K}\right]\end{array}$ & $\begin{array}{l}\text { Thermal conductivity } \\
\text { at } 600^{\circ} \mathrm{K},\left[\mathrm{W} /\left(\mathrm{m}^{\circ} \mathrm{K}\right)\right]\end{array}$ \\
\hline Alumina Silica Fibre & 0.35 & 42.7 & 0.09 \\
\hline Mineral Wool Fibre & 0.165 & 56.2 & 0.1 \\
\hline
\end{tabular}

Table 3.1: Summary of the GEN IV insulation based on SCWL [18]

$$
U=\frac{1}{\frac{1}{\bar{h}}+\frac{1}{h_{\text {wall }}}}
$$

$\bar{h}$ is average convection coefficient on the external surface of the plenum. Based on overall equivalent convection coefficient the convection coefficient is computed value of $0.5 \mathrm{~W} / \mathrm{m}^{2 \circ} \mathrm{K}$ for the thinner part of the plenum and it was applied on the outer surface of the plenum.

The ramping up time for inside AT is 180,000 seconds that varies linearly in time. 
After start up and shut down there is a delay time to give time to the domain to reach steady state. Settling time is about 280,000 $(s)$. The shut down time and second settling time is similar to startup time and first settling time respectively. Fig 3.3 shows the minimum and maximum temperature after solving the energy equation for one cycle.

Time steps for applied pressure and temperature are the same (See the Appendix C for discussion on how to choose time step size). The time step was set to be 10000 $(s)$ for a total time of analysis of 920,000 $(s)$. VrSuite does sub time stepping. The result was consistent with choosing time steps based on based on the Fourier number:

$$
F_{O}=\frac{D t}{l^{2}} \geq 1 \longrightarrow \Delta t \geq \frac{D t}{l^{2}}
$$

A Neuman pressure boundary condition is applied to the inside surface of the plenum. The pressure ramping up time is same as the thermal problem. The same time stepping was set. The pressure ramped up from $5 \mathrm{MPa}$ to $25 \mathrm{MPa}$. The same pattern of startup and shut down procedures as thermal BCs were used for stress

BCs. The total time for the stress problem is the same as thermal problem. The maximum number of Newton-Raphson iterations in a time step was set to 15 and the convergence tolerance was set to $10 e-4$. Initial temperature of the domain is 300 ${ }^{\circ} K$.

\subsubsection{Governing equations}

Thermal and stress governing equations used as follows:

The heat equation is based on the conservation of energy principle [19]. 


$$
\begin{aligned}
& \dot{h}+\nabla \cdot q-Q=0 \\
& q=-\kappa \nabla T \\
& d h=c_{p} d T
\end{aligned}
$$

where $h$ is enthalpy, $\kappa$ is the heat conduction coefficient (matrix), $q$ is the flux, $c_{p}$ is specific heat, $Q$ is the heat generated per unit volume. and $T$ is the temperature.

The governing equation for stress is:

$$
\begin{aligned}
& \nabla \cdot \sigma+b=0 \\
& \sigma=D \varepsilon \\
& \varepsilon=\left(\nabla u+(\nabla u)^{T}+(\nabla u)^{T} \nabla u\right) / 2
\end{aligned}
$$

The first equation above is the conservation of momentum equation, where the inertial term $\rho \frac{\partial v}{\partial t}+v \cdot \nabla v$, with a density field $\rho$ and a velocity field $v$, is ignored. The second and third equations are the stress-strain constitutive the relation and relation between displacement and strain. In the above governing equations, $\sigma$ is the Cauchy stress tensor, $D$ is the $6 x 6$ matrix for the elasticity tensor, $\varepsilon$ is the GreenLagrange strain tensor, and $b$ is a body force. Green-Lagrange strain equality is the compatibility equation in which the strain is the symeterized spatial gradient of the displacement. 


\subsubsection{Stress analysis}

The supports for the plenum are designed in the way that they provide a flexible joint. Due to high stress around supports that cause stress concentrations in the vicinity of joint between the plenum and supports, the supports are removed. Instead, two perpendicular planes in $x$ and $y$ direction are defined. $z$ displacement in a plane between hot and cold nozzle. The displacement in all the nodes sufficiently close to the mentioned plane were constrained with soft spring (stiffness $1 \mathrm{~N} / \mathrm{m}$ ) that constrains the displacement of nodes in the direction of their fixities.

\subsubsection{Displacement and effective stress}

The displacement of the plenum for one complete startup-shutdown cycle is shown in the Fig 3.4. The maximum displacement occurs where the hot nozzles join the pipes. The plenum tries to bend so the maximum distortion happens in the middle of plenum where the hot nozzles are located. The displacement could be added to the pipes as a Dirichlet boundary condition. The other place which has the most displacement is top of the plenum.

Fig 3.5. shows the effective stress in the plenum (Appendix B discuses the computation of the effective stress and the effective plastic strain ). The regions with high effective stress are more susceptibility for plastic flow and crack initiation. The maximum displacement and effective stress happens at time step 20 and 14 respectively. High effective stress regions are: top of the head of the plenum, the outer fillets of the hot nozzles, the inner edge of the hot nozzles and the corner that joins the plenum to the tube sheet. 
The cumulative effective plastic stress was computed for ten cycles. The isometric view of EPS after 10 identical cycles is shown in Fig. 3.6 a). However, the Maximum EPS happens at the inside edge of nozzles Fig. $3.6 \mathrm{~b}$ ). After 10 cycles the outside point on the nozzles and the top head will ratchet. The point that connects the tube sheet to the plenum will shake down (See Fig. 3.7). 


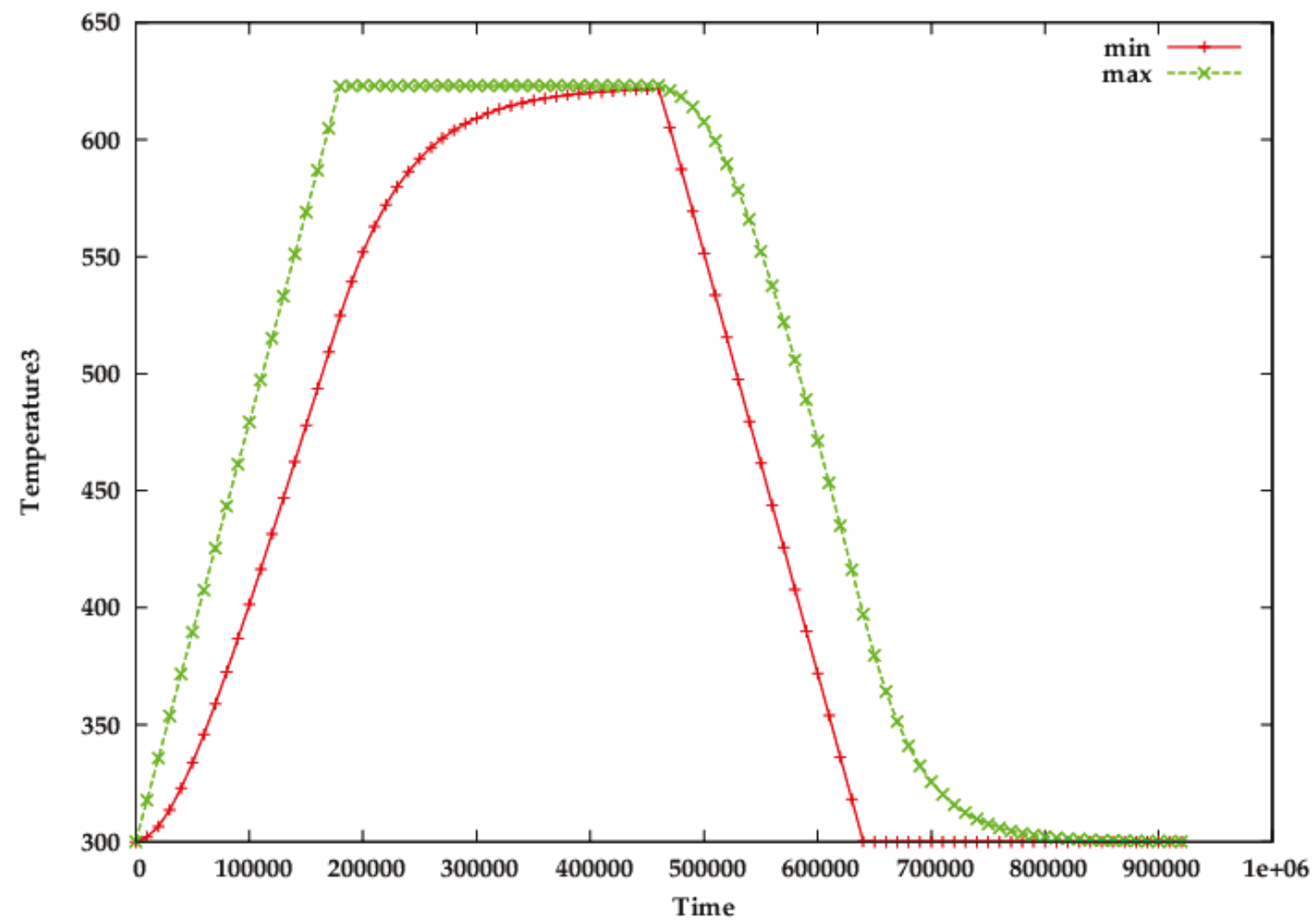

Figure 3.3: Maximum and minimum temperature in the plenum after completing one complete thermal cycle. 


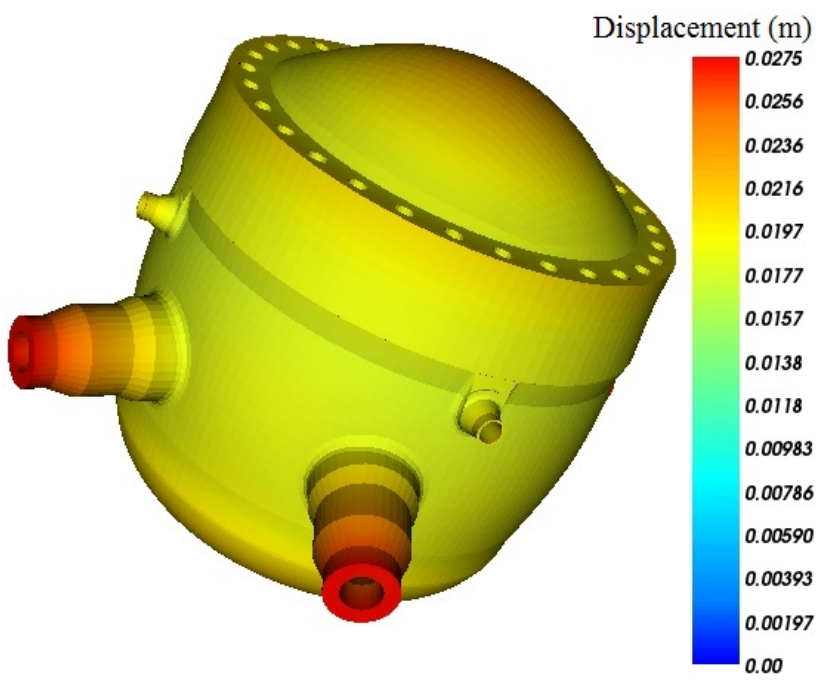

A)

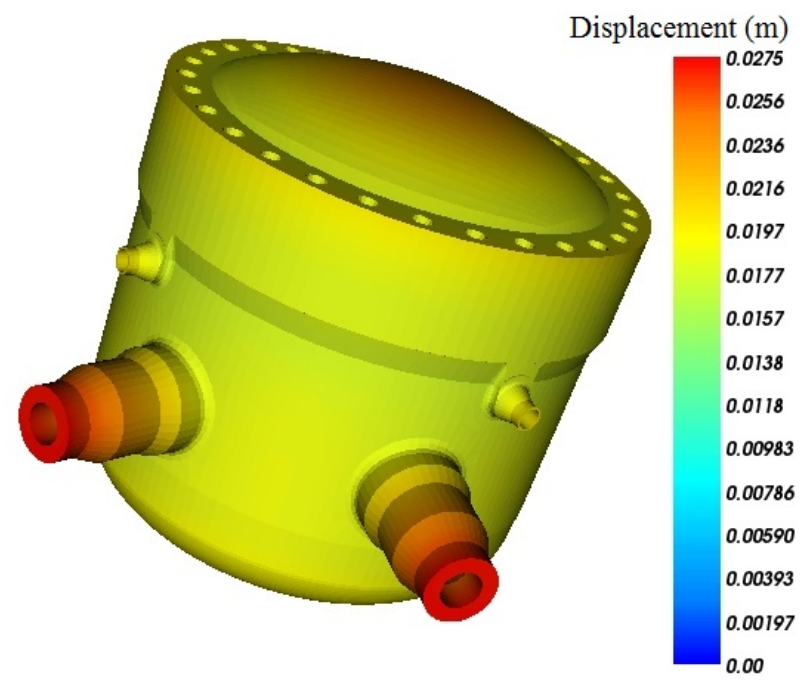

B)

Figure 3.4: Maximum displacement of plenum A) Deformed geometry B) Undeformed geometry

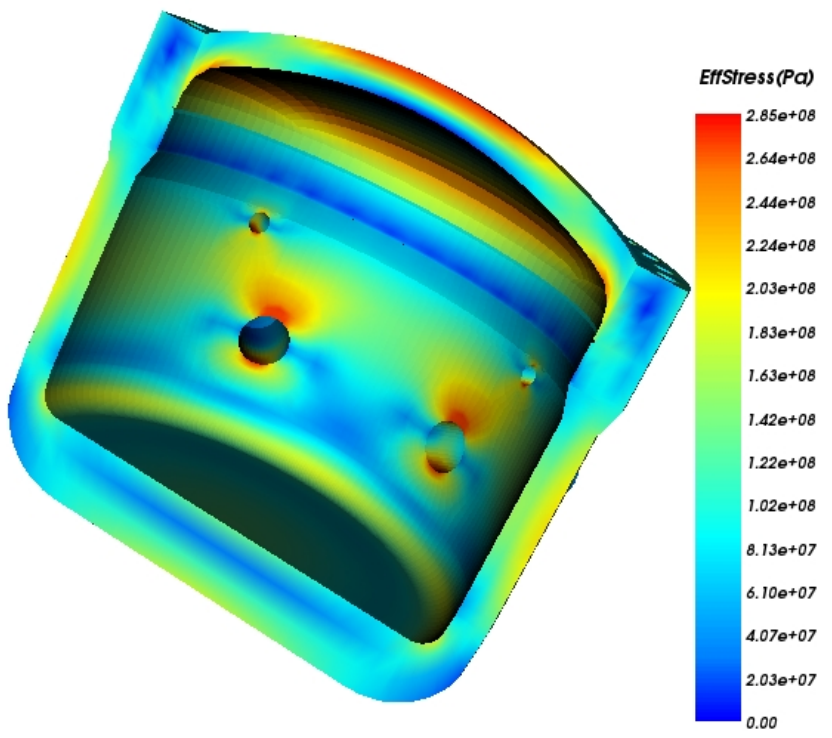

A)

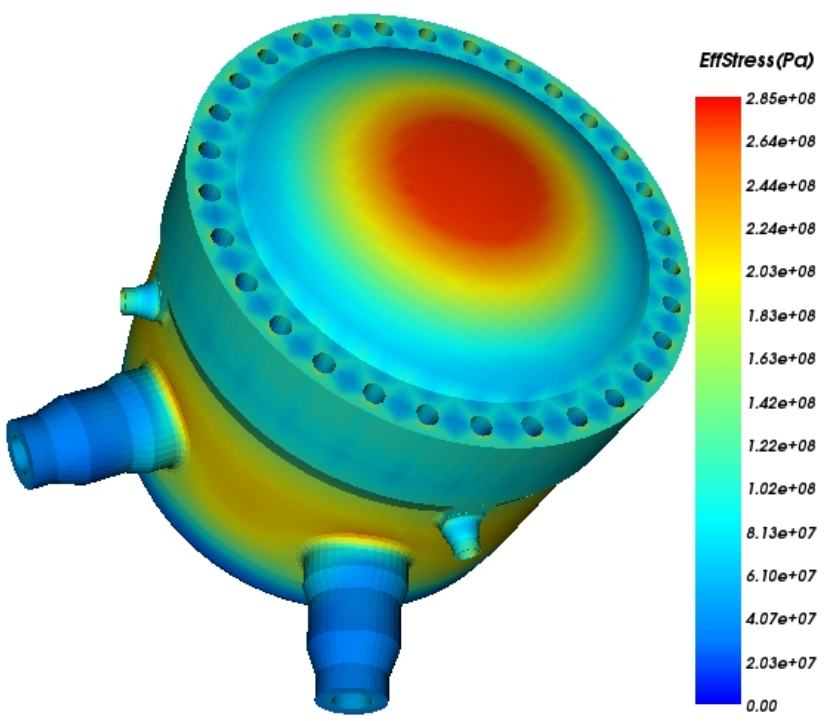

B)

Figure 3.5: Maximum effective stress A) Cut plane view B) Top view. 


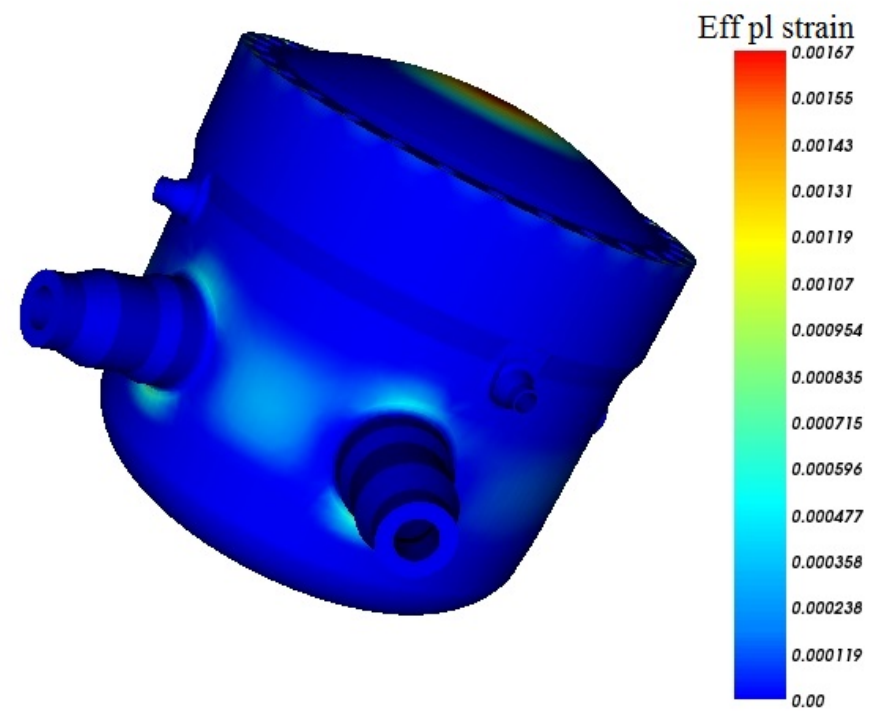

A)

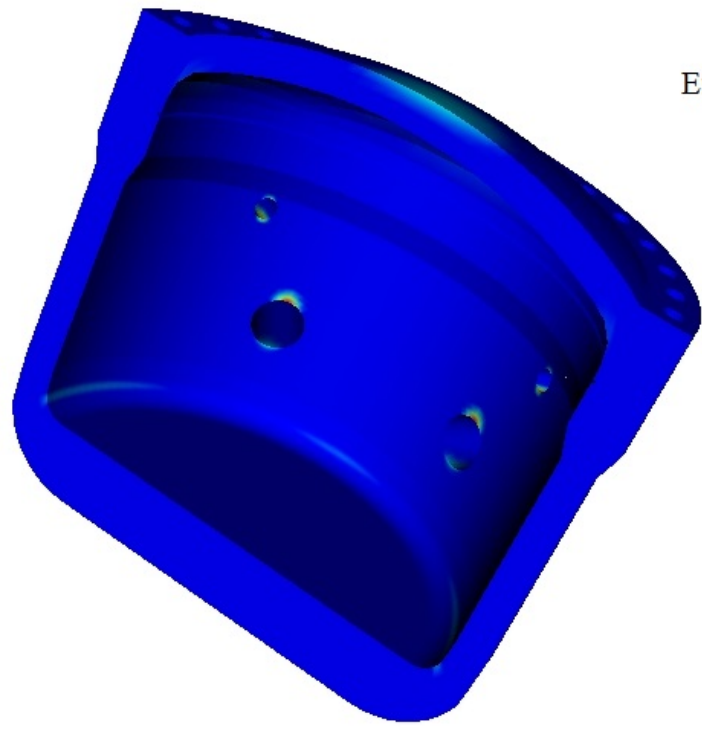

B)

Figure 3.6: Maximum EPS A) Isometric view after 10 cycle. B) Section view of the reactor plenum after 1 cycle.

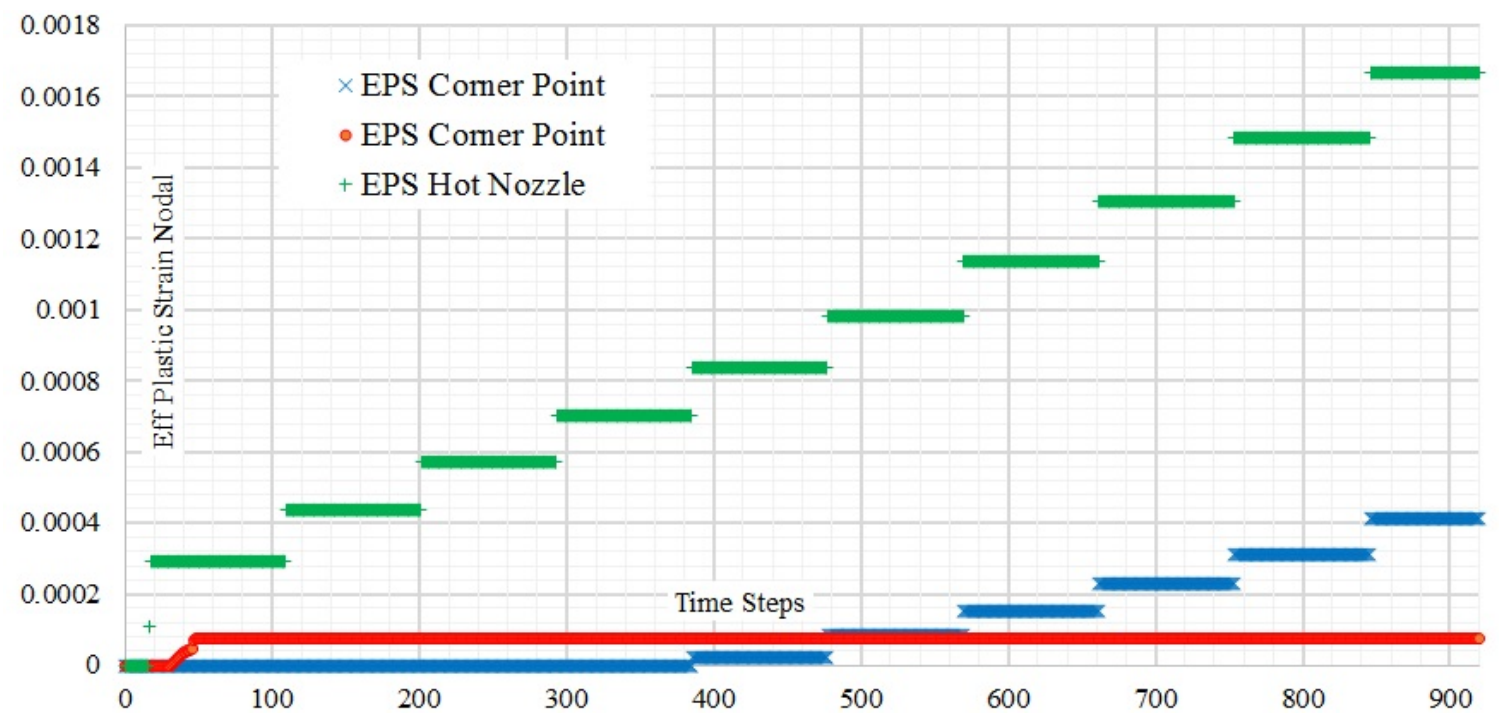

Figure 3.7: EPS for three points after 10 cycles. 


\section{Chapter 4}

\section{Optimization Of Temperature Profile Of Startup-Shutdown}

\subsection{Introduction}

Reactors experience multiple start up and shut down cycles during their operational lives. Depending on how fast the reactor temperature and pressure heat up and cool down, the fatigue load intensity will change. There are many published papers which discuss the thermal analysis of fast reactors (FR) and its affect on the reactor core design. Based on start up procedures for supercritical fossil fired power plants (FPP) and light water reactors (LWRs), supercritical pressure water reactor (SCWRs) start up systems have been developed. Basically there are two kinds of start up systems: Constant pressure start up and sliding pressure startup. Each startup method needs its own equipment and requirements to change the flow from the bypass system to the once-through line. In a constant pressure method, the reactor starts at supercritical 
pressure, and the reactor bypass system is equipped with a flash tank and a pressure control valve. In the sliding pressure method, the boiler and the reactors work at sub-critical pressure and a steam water separator and drain tank are required [20].

The constant pressure startup method is based on FPPs and it has some characteristics which are:

- This system has a complex switching valve that starts at super critical pressure. In this method, nuclear heating starts after supercritical pressure is provided by pumps. The core of the reactor is depressurized by flowing the steam into a flash tank. Saturated steam goes to a heater and the extra steam goes to a condenser. If the pressure of the flash tank is adequate then the steam goes to the turbines [20]. Fig. 4.1 shows the schematic of a constant pressure startup system. The stages of constant pressure startup system are depicted in Fig 4.2.

In the sliding pressure start up method the pressure for starting the nuclear heating is sub-critical and a steam water separator is needed (See Fig 4.3). A sliding pressure startup systems does not need super heaters so they are more economical in comparison with constant pressure ramping up systems. One of the most novel idea in sliding start up systems is proposed by Sutanto et al. [21].

Start up systems are designed in two ways in SCWR:

- Using the by pass system of FPPs. This system is divided into two classes. One which uses a recirculation pump and the other that uses an additional heater instead of a pump. In this system the power remains constant while the pressure is increased by the pumps. Turbines will work at sub-critical pressure 
with supercritical steam which are provided by boilers. There are some thermal limitations in this start up scenario:

1. The maximum cladding surface temperature (MCST) is a constraint. So the power should be constant and low enough to satisfy this constraint. Also the flow rate should be high enough to avoid boiling in the fuel rods. Boiling depends on pressure, temperature and flow rate. When the flow rate reaches $28 \%$ of maximum flow rate the nuclear heating will be started. The core power is less than $20 \%$ of its total power when the turbines start to work.

2. The steam which enters the turbines, is saturated steam (the enthalpy should be high enough into the sub-critical pressure). Meanwhile, the flow rate should be minimized to minimize the level of water and steam in the separator.

- Using recirculation systems similar to boiling water reactors (BWR). This system is separated from the main line. The pressurization is a function of power. The recirculation system contains a steam drum, a heat exchanger and a circulation pump. See Fig. 4.4 and Fig 4.5. The flow rate is constant during the start up procedure. MCST is still a constraint in this system. However, there is no limitation for the inlet steam turbine (inlet steam is supercritical steam at supercritical pressure).

Sutanto et al. used a 1D analysis of the water path through the plenum. They solved conservation of mass, energy and momentum equation and by calculation of stress, they calculated the steam drum diameter, $D$ and its wall thickness $t$. 


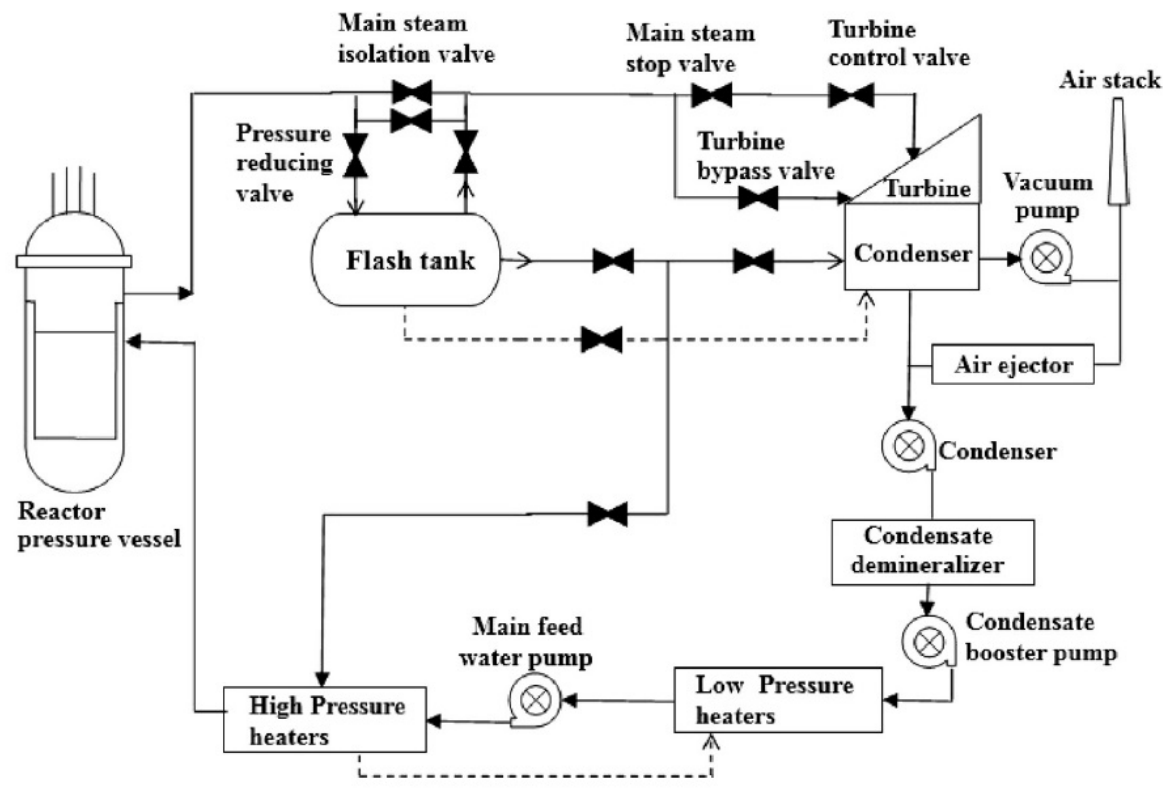

Figure 4.1: Constant pressure startup system for HPLWR. [22].

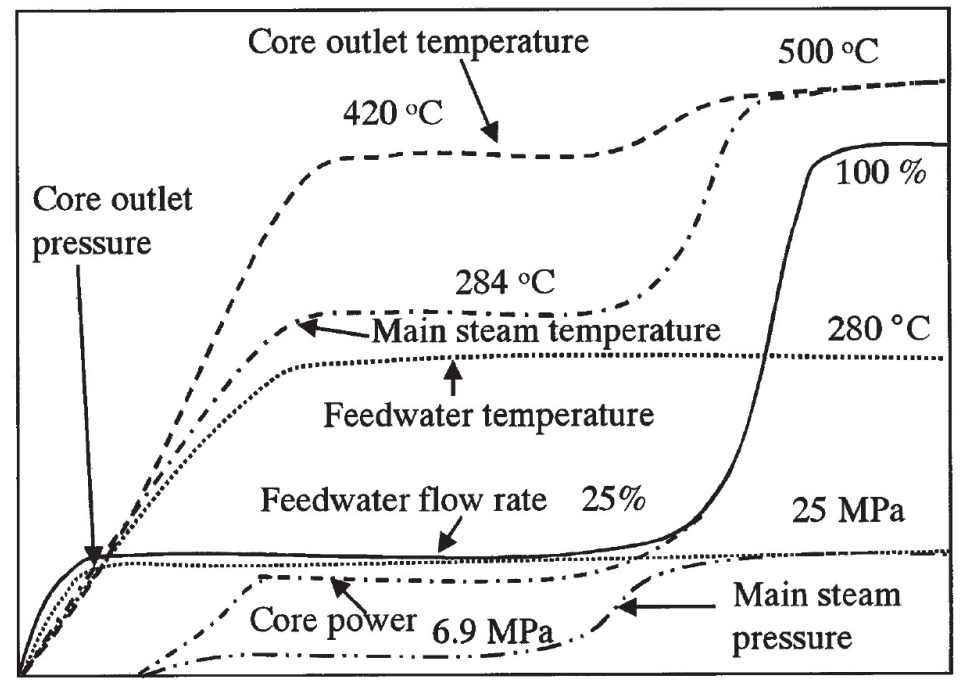

(a)

(b)

(c)

(d)

(e)

Time

Pressurization

(Startup of

Start of Turbine nuclear startup

Line

Power

feedwater pump) heating

Switching

raising

Figure 4.2: Constant pressure startup curve for SCLWR-H. [23]. 


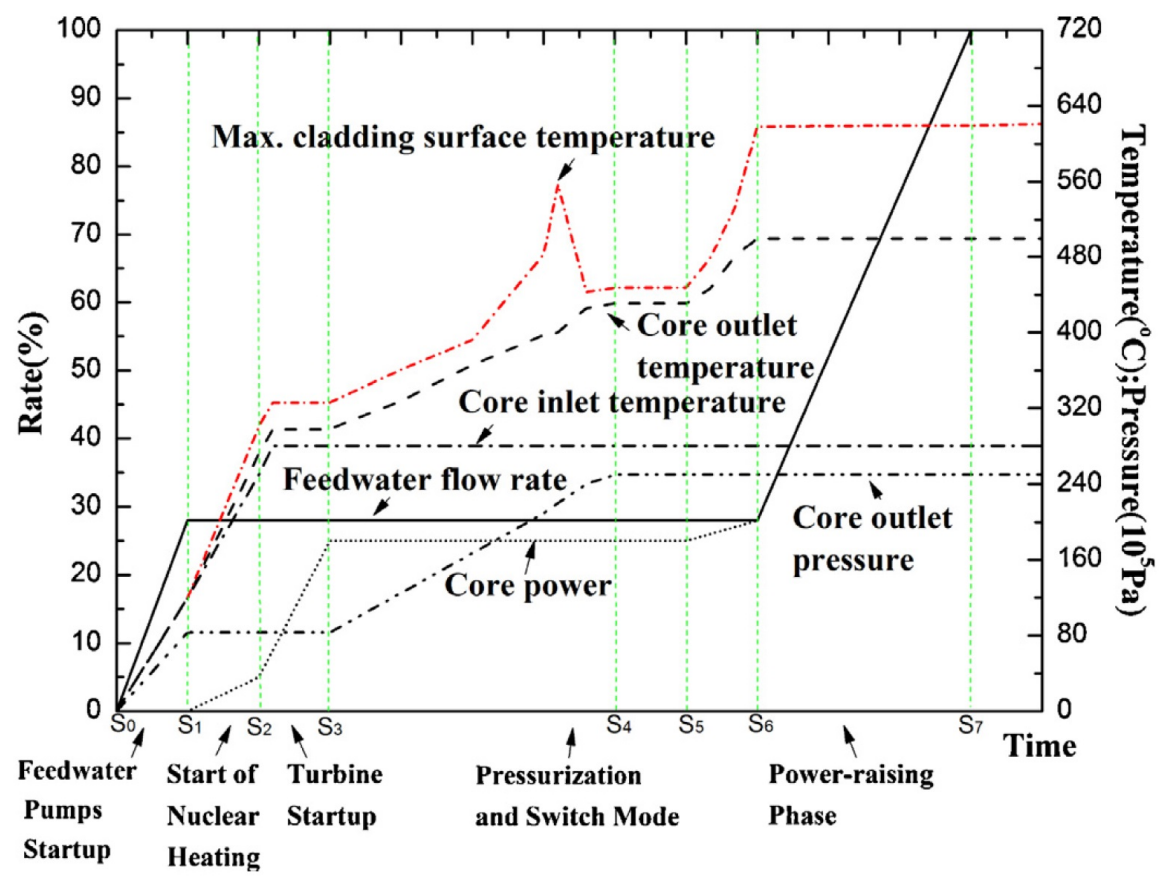

Figure 4.3: Sliding pressure startup curve for HPLWR. [22].

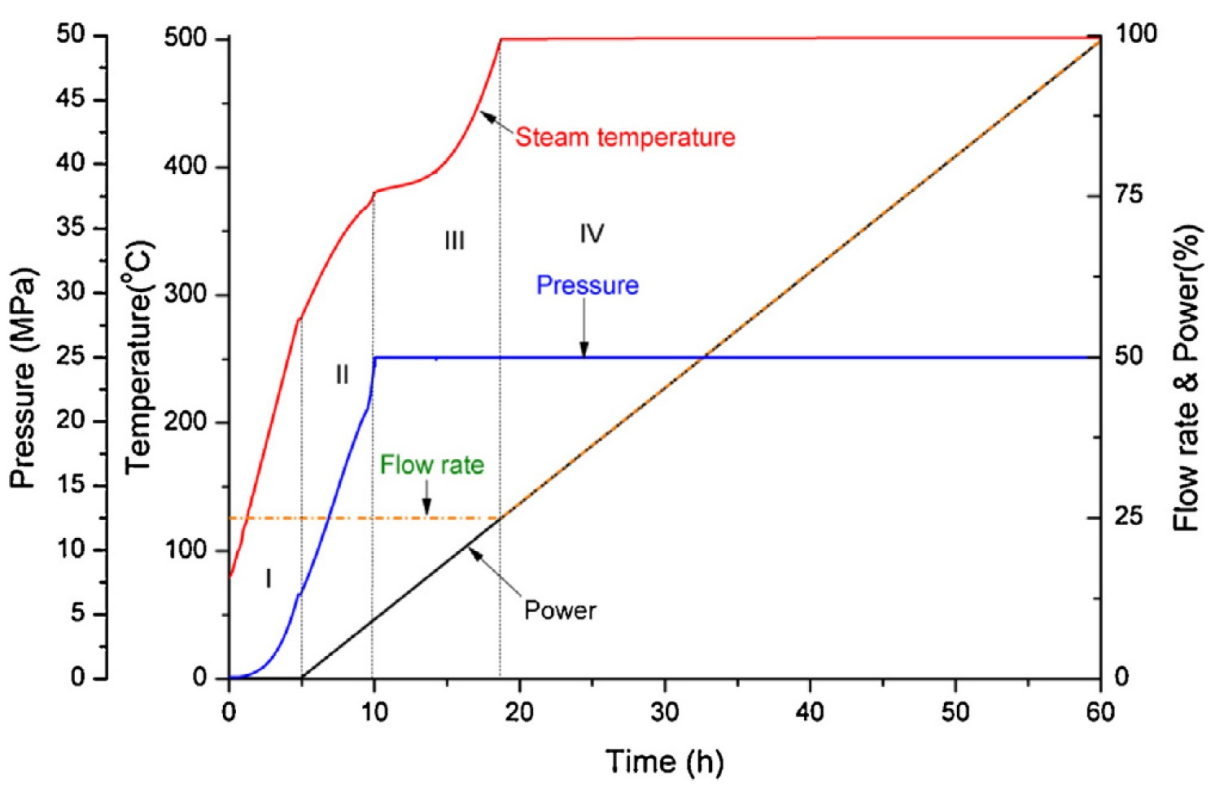

Figure 4.4: Start up stages for FR with bypass system [21]. 
Sutanto et al. classified the start up procedures into four distinct regions. (See the Fig. 4.4) :

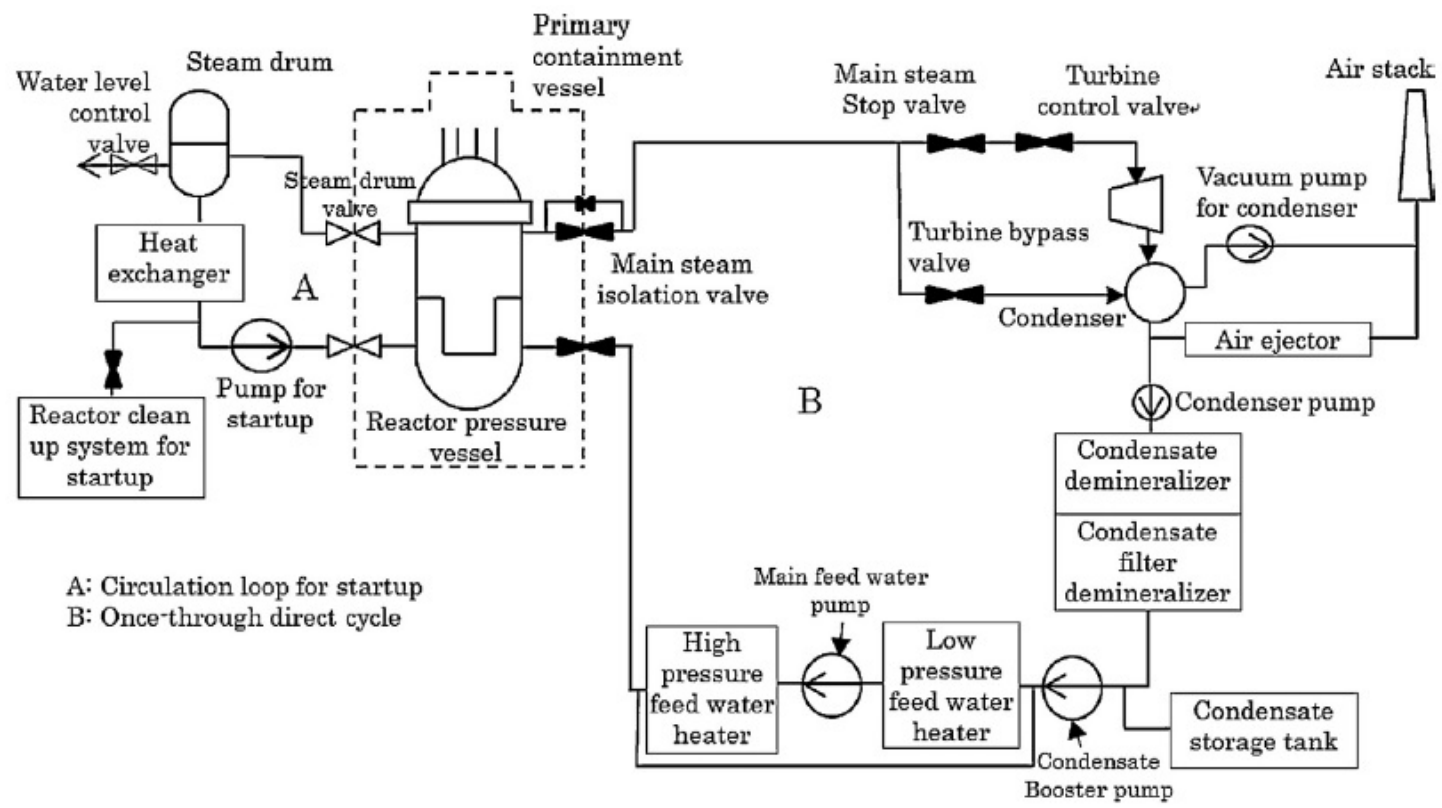

Figure 4.5: By pass system in FR which feeds the main reactor and the path of water to turbines [21].

$i$ Temperature rising: The water temperature rises from $80^{\circ} \mathrm{C}$ to $280^{\circ} \mathrm{C}$ with constant small power. After passing a maximum temperature the heat exchanger keeps the water temperature constant. The pressure increased from $1 \mathrm{~atm}$ to almost $5 M P a$.

ii Pressurization: Pressurization is done by nuclear heating in this stage. The steam drum pressure increased by nuclear power. Stabilizing the sub-saturated pressure in the core was done by maintaining steam drum water level at $3 \mathrm{~m}$. After reaching to the critical pressure $(22.1 \mathrm{MPa})$, the steam drum water level control valve will close. (It will cause a rapid change of pressure to reach $25 \mathrm{MPa}$ ). 
iii Switching from the primary start up mechanism to the main reactor: The pressure remains constant at $25 \mathrm{MPa}$. The temperature increases from $380^{\circ} \mathrm{C}$ to $500^{\circ} \mathrm{C}$. Also the power increases linearly. In this stage, the main steam stop valve and turbine control valve are opened. At this moment the by pass system is disconnected from the reactors. In all three mentioned steps, the flow rate remains $25 \%$ of total power.

$i v$ Final power raising: power is raised to $100 \%$. Flow rate tracks the power line in this stage.

Sutanto proposed two issues in the thermal analysis of start up system: a) Heat flux margin: To avoid dry out, the generated heat flux should be less than critical heat flux $(\mathrm{CHF})$. They illustrated that, there is safe margin between the CHF and generated heat flux. b) Start up sensitivity: All components of the fuel rod have a lower temperature than MCST. Also by increasing the flow rate, the MCST and the pressure decrease. Although, the flow rate should be low enough to minimize the level of water in the steam separator. It's obvious that as the inlet temperature drops the pressure decreases too.

\subsection{Problem definition}

Based on the information that is provided by AECL, the required useful life of a plenum is defined to be roughly 80 years. Due to maintenance and overhaul requirements, the reactor is expected to experience approximately three start-up and shut down cycles per a year (a total of 240 cycles in its life time). Each day of lost power 
will cost about $\$ 1 \mathrm{~m}$ for a nuclear power plant. So doing start up and shutdown as quickly as possible is one of the priorities for nuclear power plants. However, there are some physical limitations. Starting up and shutting down too fast will increase damage to the plenum and shorten its life. So there is a trade-off between starting up fast and minimizing the growth of damage in the structure. Depending on the magnitude of mechanical and thermal load that is caused by startup shut down cycles, the structure will experience damage due to elastic or plastic deformation. So there is a need to minimize the risk of producing a crack in the reactor body. To minimize this risk, optimization of the profiles of applied pressure and temperature versus time (for a fixed start up time of temperature and pressure) to minimize the rate of growth of damage should be done. The assumptions for this optimization are given below:

\subsubsection{Optimization requirement}

To define an optimization problem there is a need to set an objective function, design variables and equality and inequality constraints. There a procedure for optimization that is followed:

\subsubsection{Design space and design of experiment matrix}

The initial step in each optimization process is providing a definition for the design space. Each design variable in the design space is defined by its upper bound and lower bound values and a minimum resolution. The number of design variables define the dimension of the design space for the optimization problem.

Each discrete optimization process can be characterized by a design of experiment matrix (DOE matrix). The DOE matrix is a $m \times n$ matrix, where $m$ denotes the 
number of analyses or numerical experiments and $n$ denotes the number of design variables as parameters. In this thesis, each row represents a FEM analysis with $n$ design variables. Each row in this matrix has one value of the objective function.

The optimization of the profile of applied pressure and temperature versus time will be investigated for the GEN IV plenum. The temperature and pressure are applied as BCs (Convection coefficient, ambient temperature and Neumann pressure) on the inside surface of the hot plenum. The details of each boundary condition will be discussed in BC part for each problem. The ambient temperature (AT), convection coefficient and pressure are ramped up in start up. In the examined optimization problem, each ramping up profile of temperature and pressure is described by two piecewise linear functions. Each ramped up profile (in a general case) is defined three points. The first and the last points are constrained to the cool down state and the operating state respectively (These points are not in the design space. They are bounds on operating temperature and pressure) and the middle point is free to move between upper and lower bounds in the design space. This is demonstrated more clearly in Fig. 4.9 for startup pressure and AT ramp up that represents the design space for pressure, temperature and time for ramping up. The design variables of the middle point varies in 2 dimensions, i.e., it has 2 degrees of freedom (DOFs) for pressure (pressure and its time) and also 2 DOFs for temperature (temperature and its time). So in a general case, this optimization problem will have a 4D design space. These four design variables are: Ambient temperature in convection $\mathrm{BC}$ and the pressure for the middle point and their associated time. However, it will be explained that if the operators choose to make pressure a function of temperature, the optimization problem will be a 2D problem (two pressure parameters are a function 
of the temperature parameters).

Based on the design that will be assumed, the upper bound and lower bound of pressure in the design space and temperature will be different (In each optimization scenario it will be described separately). In the mentioned optimization process for a plenum, the DOE matrix dimension is $m \times 4$, while $m$ varies with the number of analyses that the user defines. The choice depends on the method of optimization.

\subsubsection{Objective Function}

To pose an optimization problem, at least one objective function should exist. The optimization process tries to optimize the value of the objective function. In fact, it solves one value of each objective function for each row of the DOE matrix, i.e., it does one FEM analysis and reports the value of the objective function for that row with respect to the values of the design variables in the row. The optimum value for an optimization problem will be chosen from the reported values of the objective function. The objective function which is minimized throughout the process of optimization is EPS that is measured at the end of one complete cycle of start up and cooling down. Two cases are considered for all analyses: a) minimizing the maximum EPS in the domain, i.e., the complete structure; b) minimizing the EPS for a specific point of

plenum. As the EPS is non-decreasing in time, the EPS for one cycle is computed and set as the value of objective function.

\subsubsection{Constraints}

There are some constraints that are defined for optimization problems. The shut down profile is constrained to the startup profiles. In other words, the shut down 
profiles are set in a similar way so that absolute value of the slope of the line equals the slope of ramping up. After start up and shut down there is a delay time that allows the domain to reach steady state. The delay time value has been considered to be fixed and its value for start up is the same as the one for the shut down settling time value.

So any optimization process can be defined with four requirements:

1. Objective function: A function of design variables that will be minimized by the optimization processes.

2. A vector of optimization variables: Each optimization problem has set of design variables which changes to minimize the objective function.

3. The upper and lower bound for each design variable: Each design variable, has an upper bound and lower bound. Design variables change within the range that is defined by the upper and lower bound.

4. Equality and inequality constraints: In addition to the upper and lower bound on each design variable, equality and inequality constraints can be defined that governed by physics and initial conditions of the optimization problem. Conservation of mass, momentum and energy are possible equality constraints. Bounding the stress to be less than the yield stress is a possible inequity constraint.

Below is the mentioned requirements in mathematical form: 
$F(\mathbf{X})$ (Objective function that is function of vector of design variable $\mathbf{X}$ ) (4.1)

$$
\begin{array}{ccr}
g_{j}(\mathbf{X}) \leq 0 & j=1, m & \text { (inequality constrain) } \\
h_{k}(\mathbf{X})=0 & k=1, n & (\text { inequality constrain) } \\
X_{i}^{l} \leq X_{i} \leq X_{i}^{u} & \text { (Upper and lower bound of each variable } \left.\mathbf{X}_{\mathbf{i}}\right)
\end{array}
$$

In the problem that is defined below the temperature $\left(T_{i}\right)$ and time $\left(t_{i}\right)$ are considered as design variables. The objective function is EPS. The pressure boundary condition is an equality constraint. Also for each case the upper and lower bound of each variable is well defined.

\subsubsection{Methods of Optimization}

The optimum obtained by optimization could be global or local which are defined in the following section:

\subsubsection{Global Optimization}

VrSuite allows global optimization to be conducted in two ways:

1. Full grow: It will find the optimum value by searching the complete discrete design space. It needs the minimum and maximum value of each design variable in the design space and the resolution intervals that are a power of two. Refining the grid will result in a more accurate value of optimum.

2. Kriging: The Kriging ( [24], [25] and [26]) seeds a number of points that is set 
by the user for the first iteration based on statistical considerations, e.g., LATIN hypercube. In the next iteration Kriging will seed points in the design space in the vicinity of local optimum. A linear combination of a polynomial function and Gaussian distribution function at each design point will be fitted through the point. The purple stripes in Fig. 4.12 shows the uncertainty adjacency of each design point. The Kriging algorithm tries to minimize the uncertainty in the global problem in each iteration by putting more design points to detect and correct the behavior of the fitted surface. Finally it will report the global optimum.

\subsubsection{Local Optimization}

There are several ways to find local optimum in the VrSuite. To define such an optimization problem, one must define the initial value and bounds on the increments of marching through the design space.

\subsubsection{FEM analysis}

To report the objective value for each row of the DOE matrix, a thermal and stress FEM analysis of the plenum is done. Thermal analysis will be done and subsequently input the temperature field as a data flow for stress analysis. Stress solver will compute and report the EPS for each time step. The conditions for doing FEM analysis for the plenum are listed below: 


\subsubsection{Domain}

If the nozzles are removed from 3D domain, the remaining part is a 3D axisymmetric part, which can be modeled with a 2D axisymmetric domain. The 2D axisymmetric domain is considered as the domain for all optimization problems in this section. In the next part the difference between a discrete 3D axisymmetric analysis results and the result from a 2D axisymmetric domain will be discussed.

\subsubsection{Meshing and the Material properties}

The $2 \mathrm{D}$ axisymmetric domain is made of conforming quadrilateral elements that is illustrated in Fig 4.6. Mesh independent analysis is done. To resolve stress concentrations, corners are meshed with a fine mesh. The plenum material is SA533 steel.

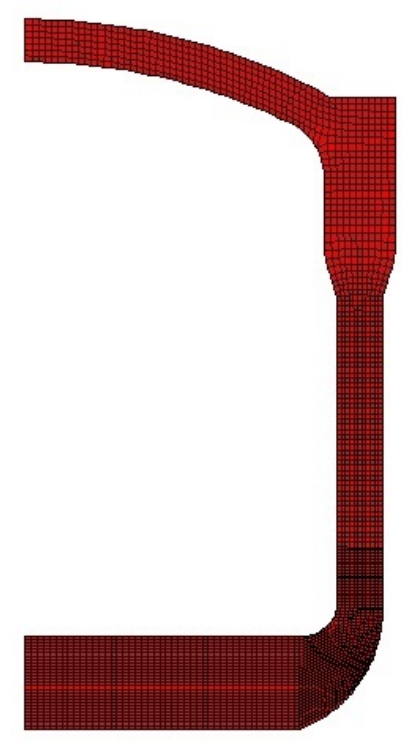

Figure 4.6: The mesh that was employed in the 2D axisymmetric mesh. 


\subsubsection{Boundary condition and Initial condition}

A Neuman pressure boundary condition is applied to the inside surface of the plenum. During startup the pressure changes from atmospheric pressure $(1 \mathrm{~atm})$ to the operating pressure $(25 \mathrm{MPa})$. For thermal analysis, the inside ambient temperature is ramped up from $300^{\circ} \mathrm{K}$ to $623^{\circ} \mathrm{K}$ in the most extreme case. (In some cases the range of the design space will shrink). The AT for the outside surface will be always room temperature $\left(300^{\circ} \mathrm{K}\right)$. The convection coefficient for the inside and outside surface is considered to be $1000 \mathrm{~W} / \mathrm{m}^{2 \circ} \mathrm{K}$ and $0.5 \mathrm{~W} / \mathrm{m}^{2 \circ} \mathrm{K}$ respectively.

In the axisymmetric $2 \mathrm{D}$ solver, the part which is adjacent to the axisymmetric axis ( $\mathrm{Y}$ direction) is fixed in the $\mathrm{X}$ direction by axisymmetry. So only one node in the middle outside of plenum is fixed in $\mathrm{Y}$ direction.

The initial condition and governing equations are the same as described in chapter 2 .

\subsubsection{Shake down Analysis}

There is a need to do more analysis to find out if the life of the plenum will exceed 240 thermal/stress load cycles. So overall, the behavior of the plenum in each circumstances should be tested to determine if the plenum is in the elastic region or not. If the plenum is in the plastic region, the designer should make sure that the points at highest risk will shake down after a finite number of cycles. After that the deformation will be in the elastic region. To capture the behavior, in addition to EPS, the displacement and plastic strain tensor should be considered too. The maximum displacement should have a descending trend. 


\subsection{Result and discussion}

A 2D axisymmetric plenum without nozzles has been chosen as a reference design for optimization. Before doing optimization, doing thermal and stress analysis on the reference design is necessary. There is difference in the results and how accurate the $2 \mathrm{D}$ axisymmetric is in comparison with $3 \mathrm{D}$ axisymmetric and the original 3D mesh. To compute the difference in effective stress and EPS between the 3D meshes and reference design mesh (2D axisymmetric mesh) a set of analyses has been done with one cycle of a linear ramped up ambient temperature and pressure. Four cases were compared: two cases with a 3D axisymetric mesh with 40 and 120 circumferential elements, a 2D axisymmetric mesh and a 3D mesh with nozzles in it. See Table 4.1 that compares the CPU time for doing stress and thermal analysis for different meshes and the number of $2 \mathrm{D}$ or $3 \mathrm{D}$ elements in each mesh. The table will justify the reason of using 2D axisymmetric mesh for startup-shutdown optimization process. Doing thermal/stress analysis on the $2 \mathrm{D}$ axisymmetric mesh is almost 70 times faster then doing it on the AECL 3D geometry.

The difference in EPS and the error of effective stress based on 2D axisymmetric mesh is presented in the Table 4.2 (Appendix $\mathrm{C}$ compares the difference of 2D, 3D axisymmetric mesh stress and thermal results with an exact solution). The reason for the difference between 2D axisymmetric mesh and 3D axisymmetric mesh with different number of circumferential elements is due to the discretization. Equation 4.5 is the element stiffness matrix for a 3D axisymmetric mesh in $(r, z)$ coordinates. As the number of elements in the circumferential direction increases, the result approaches to $2 \mathrm{D}$ axisymmetric results. Equation 4.6 represent the element stiffness for a 3D 


\begin{tabular}{|l|l|l|l|}
\hline Domain type & Numberofelement & Thermal time $(s)$ & Stress time $(s)$ \\
\hline $2 D$ axisymmetric & 3309 & 57.6 & 40.2 \\
\hline $3 D(40$ Element $)$ & 35674 & 129.7 & 848.52 \\
\hline $3 D(120$ Element $)$ & 109200 & 465.9 & 4740 \\
\hline $3 D$ mesh & 76392 & 1262 & 5306.2 \\
\hline
\end{tabular}

Table 4.1: The comparison of CPU time for doing stress or thermal analysis for different meshes and their number of elements.

axisymmetric mesh in Cartesian $(x, y, z)$ coordinates.

$$
\begin{gathered}
\mathbf{K}=\int_{\Omega^{e}} \mathbf{B}_{\mathbf{r}, \mathbf{z}}^{\mathbf{T}} \mathbf{D}_{\mathbf{r}, \mathbf{z}} \mathbf{B}_{\mathbf{r}, \mathbf{z}} 2 \pi d r d z \\
\mathbf{K}=\int_{\Omega^{e}} \mathbf{B}_{\mathbf{x}, \mathbf{y}, \mathbf{z}}^{\mathbf{T}} \mathbf{D}_{\mathbf{x}, \mathbf{y}, \mathbf{z}} \mathbf{B}_{\mathbf{x}, \mathbf{y}, \mathbf{z}} d x d y d z
\end{gathered}
$$

A comparison of EPS for two different 3D axisymmetric meshes has been shown in the Fig. 4.7. The EPS for a 2D axisymmetric mesh for the reference design (linear ramping up) is shown in the Fig. 4.8. Fig. 4.7 represents the EPS for reference design start up for 3D axisymmetric analysis for 40 and 120 circumferential elements. The same analysis was done for a 3D mesh for a design provided by AECL (Chapter 2). The maximum EPS occurred at the nozzles. However, similar to $2 \mathrm{D}$ and $3 \mathrm{D}$ axisymmetric mesh, 3 points with high EPS are captured. 


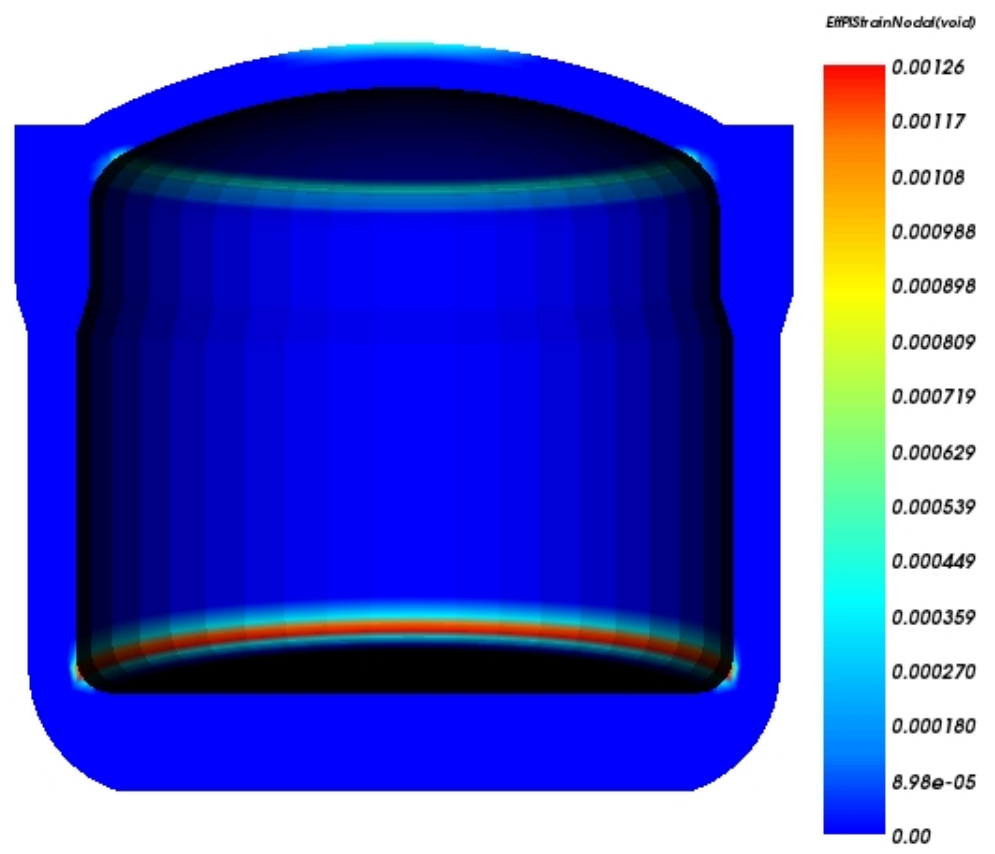

a)

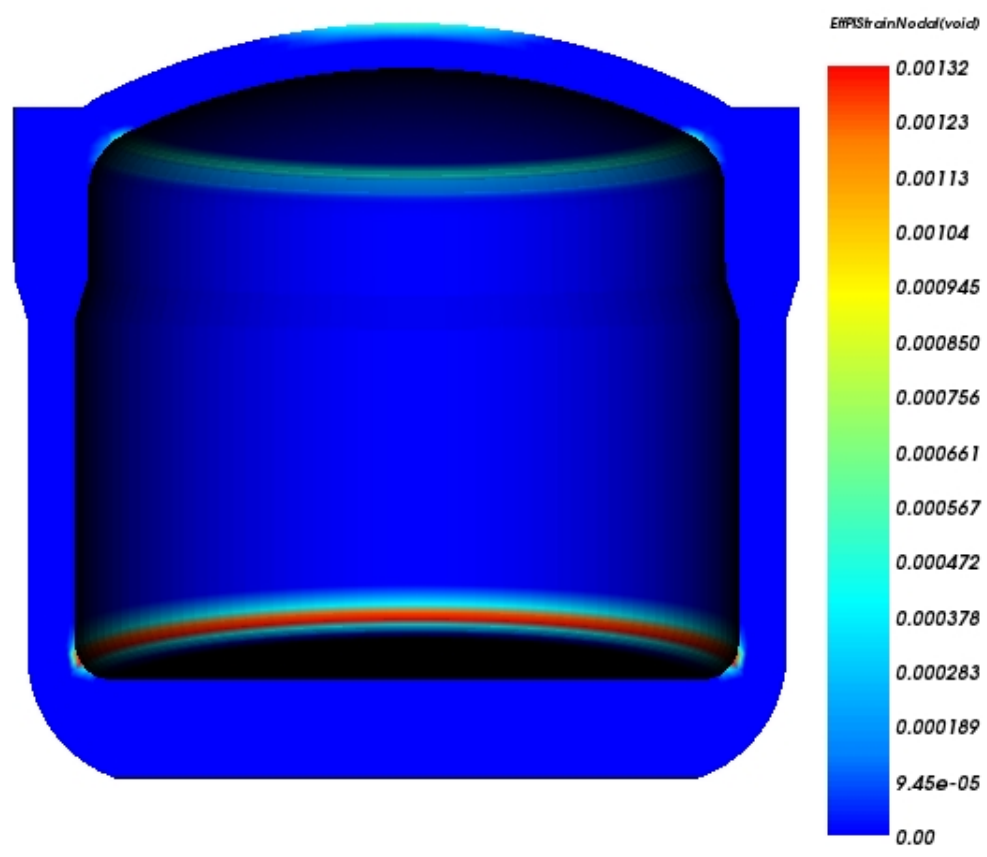

b)

51

Figure 4.7: EPS is shown as a contour plot. a) 3D axisymmetric mesh with 40 circumferential elements. b) 3D axisymmetric mesh with 120 circumferential elements. 


\begin{tabular}{|l|l|l|}
\hline mesh type & EPS difference mesh & Error in Eff stress \% \\
\hline $3 D$ axisymmetric $(40 \mathrm{El})$ & 0.00133 & $12.67 \%$ \\
\hline $3 D$ axisymmetric $(120 \mathrm{El})$ & 0.00139 & $9.86 \%$ \\
\hline $3 D$ mesh & 0.0025 & $10.67 \%$ \\
\hline
\end{tabular}

Table 4.2: The difference of EPS and error percentage based on 2D axisymmetric analysis result in effective stress of three mesh cases with $2 \mathrm{D}$ axisymmetric mesh.

\subsection{Which points in the plenum are most suscep- tible to crack initiation?}

The 2D axisymmetric of the plenum has been analyzed to predict the points that deform plastically and the point that has the most severe plastic deformation. To analyze the behavior of the plenum, a linear ramped up ambient temperature and pressure similar to the reference design analysis was applied to the inner surface of the plenum.

By comparing analyses for all the meshes and solving approaches, it was determined that the maximum EPS is located at the corner of the plenum where the main body is attached to the tube sheet and this location is independent of meshing and solver type.

Three points have been identified as the points of the design with highest risk of cracking for both 3D and 2D designs. The risk is based on the magnitude of the value of the EPS. In the basis of their EPS values the points are ordered. Point 1 has the largest EPS value (Fig. 4.8) where as point 2 and 3 have lower values. It was shown that different start up scenarios do not change the positions of these three points. So the main challenge for designing the plenum is to minimize the EPS in these three points. 


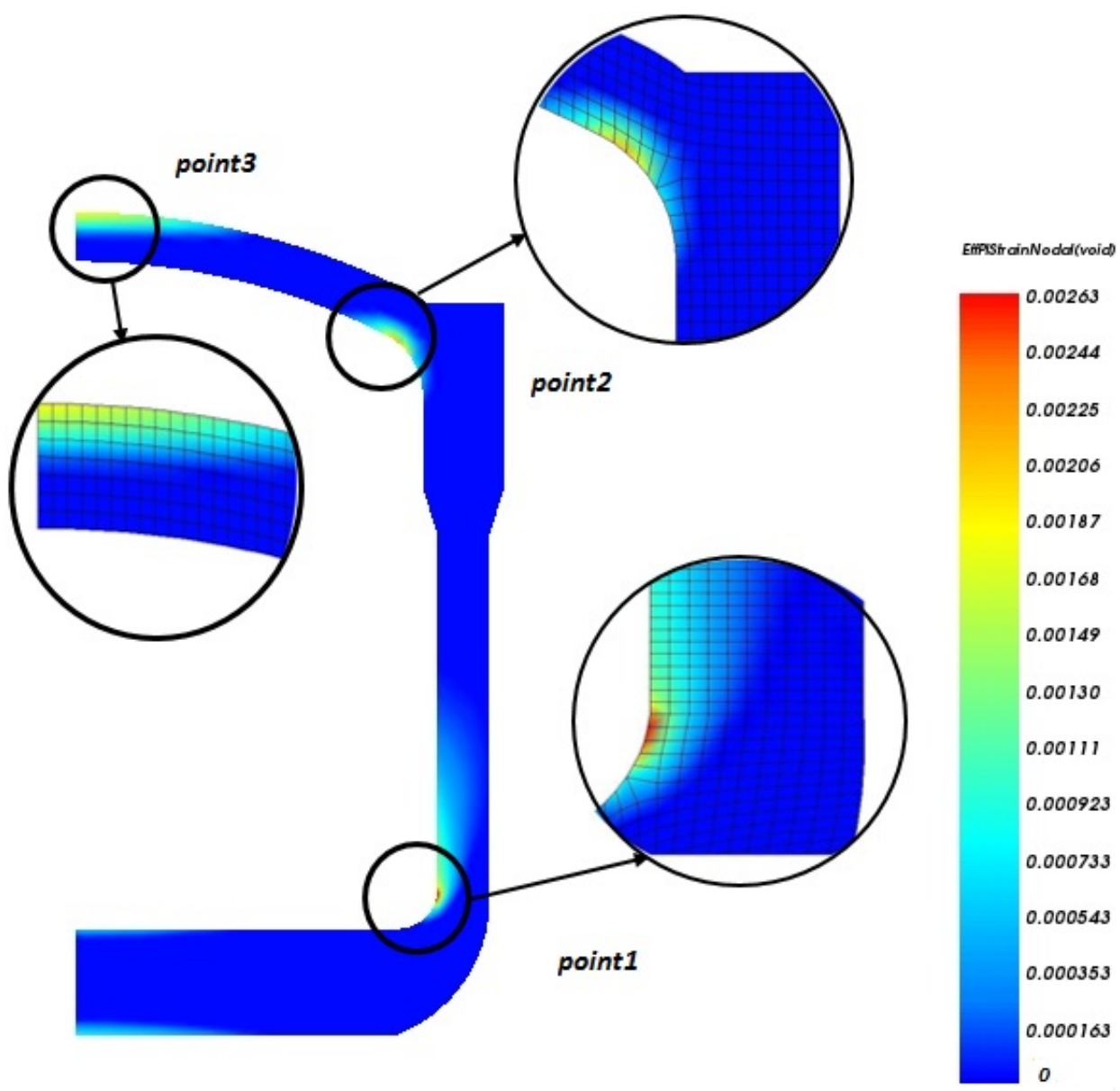

Figure 4.8: Showing the maximum EPS in the domain. Three points that have the maximum EPS are shown. 


\subsection{Optimized startup scenario}

As was discussed earlier, there are two main ways to do a start up procedure in reactors. The Sutanto, a sliding pressure start up model [21] was chosen. Water temperature is ramped up by two piecewise linear functions that are defined by three points $\left(t_{i}, T_{i}\right)$. The lower and upper bounds of DOE temperature of the thermal cycle are $310^{\circ} \mathrm{K}$ and $613^{\circ} \mathrm{K}$ respectively. The value of the design variable for ramping up time varies between $10,000 \mathrm{~s}$ and $170,000 \mathrm{~s}$ (roughly two days). Fig. 4.9 shows the design space for this problem. The design variables are $T_{i}$ and $t_{i}$. Each curve is characterized by the values of its $T_{i}$ and $t_{i}$ points. Each curve represents one point in the design space shown in Fig. 4.9. The pressure in the reactor in this method of startup is adjusted by controlling the level of water in the flash tank. This constrains the pressure. To be a function of temperature in such a way that the pressure is always $20^{\circ} \mathrm{C}$ higher than the boiling pressure. A plot of the pressure for a steam fraction equal to one is shown in Fig. 4.10 (from NIST table). To be sure that the pressure is higher than the boiling pressure, the temperature is shifted $20^{\circ} \mathrm{C}$ to the left. A cubic polynomial is fitted to the nodes in the plot and the resulting equation is the pressure boundary condition equation as a function of temperature. Both thermal and pressure use the same time stepping. A Kriging iteration has been set to five iterations and eight points were distributed in the design space for each iteration (a total of forty analyses). Fig. 4.11 shows the temperature profile and its corresponding pressure profile for several cases. 


\subsubsection{Objective Function}

The effective plastic strain (EPS) was used as an objective function. The maximum value of EPS is to be minimized at the end of a complete startup shut down cycle.

\subsubsection{The best scenario for ramping up temperature and time}

After doing the thermal and stress analysis of all forty rows of the DOE matrix and computing the objective function for each row, the optimum value of EPS was found. The optimum value of the objective function is shown in Fig. 4.12. The exact value of DOE temperature and DOE time at which the objective function value is minimum is shown in Fig. 4.12a separately. Fig. 4.12b shows evolution of objective function during Kriging alterations. It is clear that there is no need to do more than 5 than the Kriging iterations. Fig. 4.13 illustrates a 2D view of the optimized objective function where the horizontal axis is the DOE temperature and the vertical line is the DOE time. The light blue area that has a cluster of points is the region in which the optimum occurs and the dark blue is the maximum value of EPS that the designer should avoid.

\subsubsection{The effect of pressure ramping on the optimized ther- mal start up profile}

To find the effect of ramped pressure on the optimized thermal profile, the same design space for DOE temperature was chosen. However, the pressure was ramped up linearly for two different cases, one from $5 \mathrm{MPa}$ to $25 \mathrm{MPa}$ and the other one 
$10 \mathrm{KPa}$ to $25 \mathrm{MPa}$ in two days. The optimization problem tries to minimize the maximum EPS in the 2D axisymmetric domain. The boundary condition, governing equation and initial condition are the same as the former case. In the two following cases, the optimization ran five iterations. In each of Kriging iteration 8 points were seeded in the design space.

Fig. 4.14 a) and Fig. 4.16 a) express the exact value of DOE time and DOE temperature where the minimum happens for a linear ramp of DOE pressure from 5 $M P a$ to $25 \mathrm{MPa}$ and from $1 \mathrm{kPa}$ to $25 \mathrm{MPa}$ respectively. Fig. $4.14 \mathrm{~b})$ and Fig. $4.16 \mathrm{~b}$ ) shows the change of objective function for 5 iterations for the two mentioned cases. It shows that five iterations are sufficient to find the minimum. Fig. 4.15 depicts a $2 \mathrm{D}$ view of the optimized objective value and the DOE temperature and DOE time of mid point where it optimizes the maximum of EPS in the domain for the case where the DOE pressure ramped from $5 \mathrm{MPa}$ to $25 \mathrm{MPa}$. The same plot was used to show a $2 \mathrm{D}$ view of optimized objective value and the DOE temperature and DOE time of mid point for the case which the DOE pressure ramped from $1 \mathrm{~atm}$ to $25 \mathrm{MPa}$ (Fig. 4.17 )

By comparing three different ramping up scenarios, it will be concluded that, by changing the lower bound of pressure from $5 \mathrm{MPa}$ to $1 \mathrm{~atm}$ the middle point ramped pressure will approach to higher temperature and time. In other words, to achieve the least EPS the profile of temperature and pressure should be as far as possible to minimize the superposition effect on the EPS. Also by comparing linear pressure ramping up and Sutanto [21] ramping up scheme, it is concluded that, the time and temperature for a second point should be located at higher values for Sutanto [21] ramping up scenario. 


\subsection{Shake down analysis for the most susceptible points for initiation of a crack}

Because the superposition of the applied pressure and thermal load exceeds the limit of the upper bound of shake down, with any ramping up scenario shorter than two days, the point at which the maximum effective plastic strain happens (point 1), will not shake down. To find the lower/upper bound of shakedown and the maximum pressure that can shake down, ambient temperature was ramped up linearly from $300^{\circ} \mathrm{K}$ to $623^{\circ} \mathrm{K}$ and the maximum pressure which is ramped up over two days (same as ambient temperature) and is varying (See Fig. 4.18). For the case which the maximum EPS occurs (point 1) the lower bound for shake down (elastic region) was found to be $7.84 \mathrm{MPa}$. The upper bound of shake down was estimated to be 19.5 $M P a$.

Fig. 4.19 shows the EPS values versus time for point 1. To define the region for the state of loading described in the Bree diagram, the displacement of point 1 was computed. If the displacement increases in each loading cycle, it means that the domain at this point will ratchet. Figure 4.21 depicts the maximum and minimum displacement of point 1 for ten cycles. The tabular data shown an increasing displacement. If the displacement in each dimension did not increase in each cycle, but EPS did, then the point would be under going reverse plasticity. It's obvious that by changing the ramping up time and temperature, the position of point 1 in a Bree diagram will change. The approximate position of points 1, 2 and 3 in the Bree diagram will be in S2 region (See Fig. 2.6). There is a need to do a similar analysis for points 2 and 3. The results are depicted in Figure 4.20. It shows that both points 
2 and 3 shake down with a maximum $25 \mathrm{MPa}$ after 5 cycles. However, the maximum EPS is higher for point 2. The same trend was observed for the lower bound of shake down for points 2 and 3. The lower bound for shakedown is 15.05 $\mathrm{MPa}$ and $10 \mathrm{MPa}$ for points 2 and 3 respectively.

The shake down analysis is necessary for the structure integrity to ensure that structure survives the startup shut down cycles. Once this been achieved, then the designer should analyze the design to provide fatigue and creep life of 80 years. 


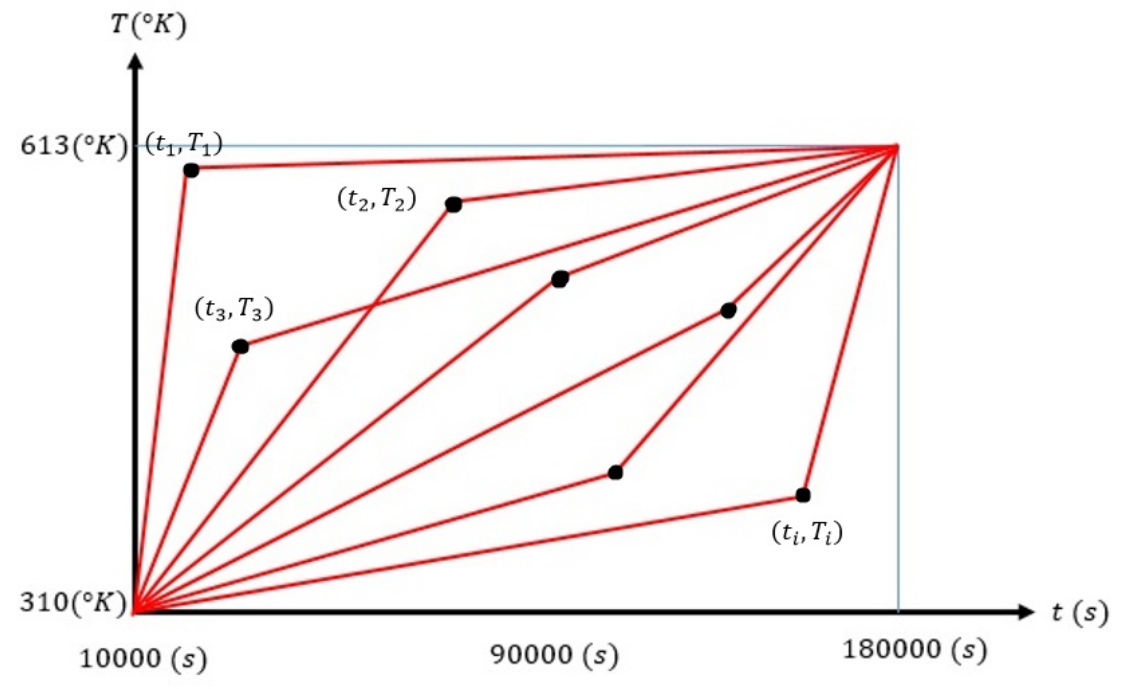

Figure 4.9: Design space for DOE temperature. Each profile is associated with one point in the design space $\left(t_{i}, T_{i}\right)$

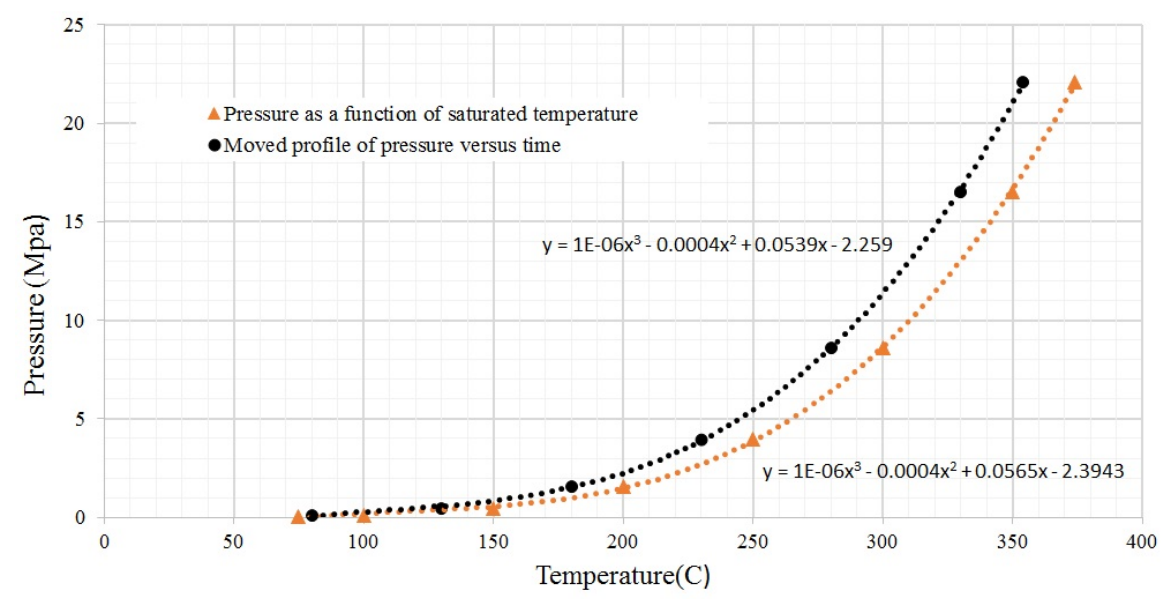

Figure 4.10: Pressure as a function of temperature with a cubic function fitted to it. The gray line is the one with temperature shifted 20 degrees to the left. 


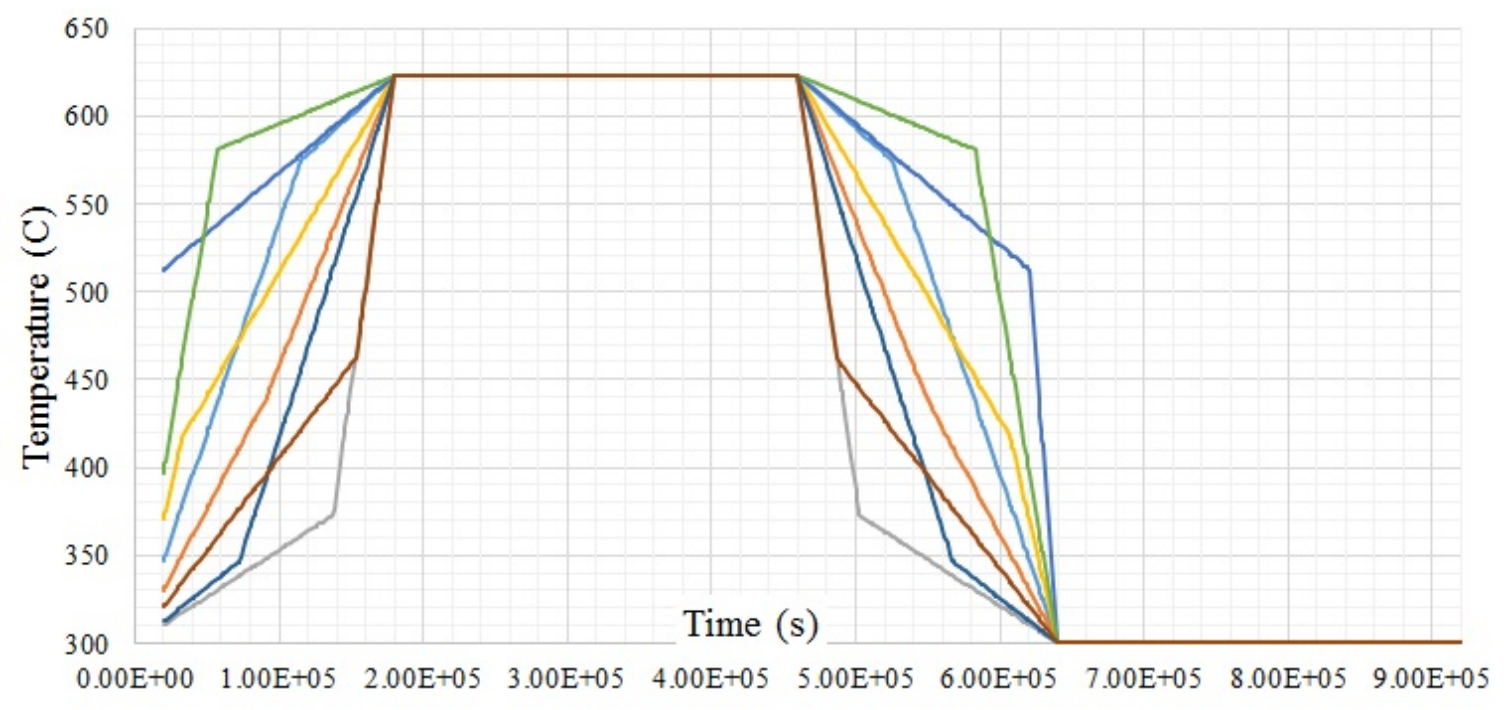

- Case1 - Case2 - Case3 - Case4 - Case5 - Case6 - Case7 - Case8

a)

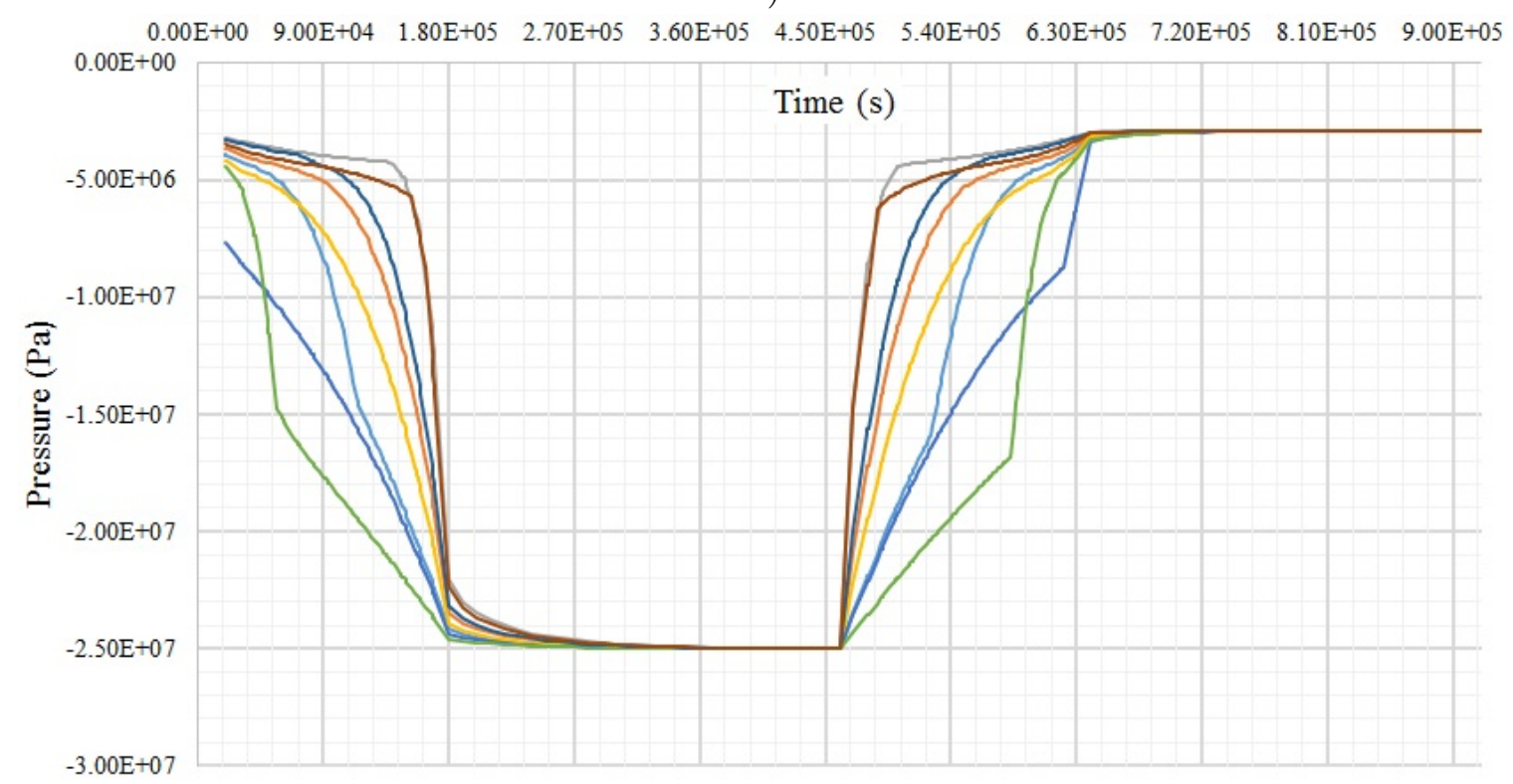

- Case1-Case2 - Case3 - Case4 - Case5 - Case6 - Case7 - Case8

b)

Figure 4.11: Arbitrary cases which shows; a) The ambient temperature applied to the inner surface of plenum. b) The corresponding pressure curve. 

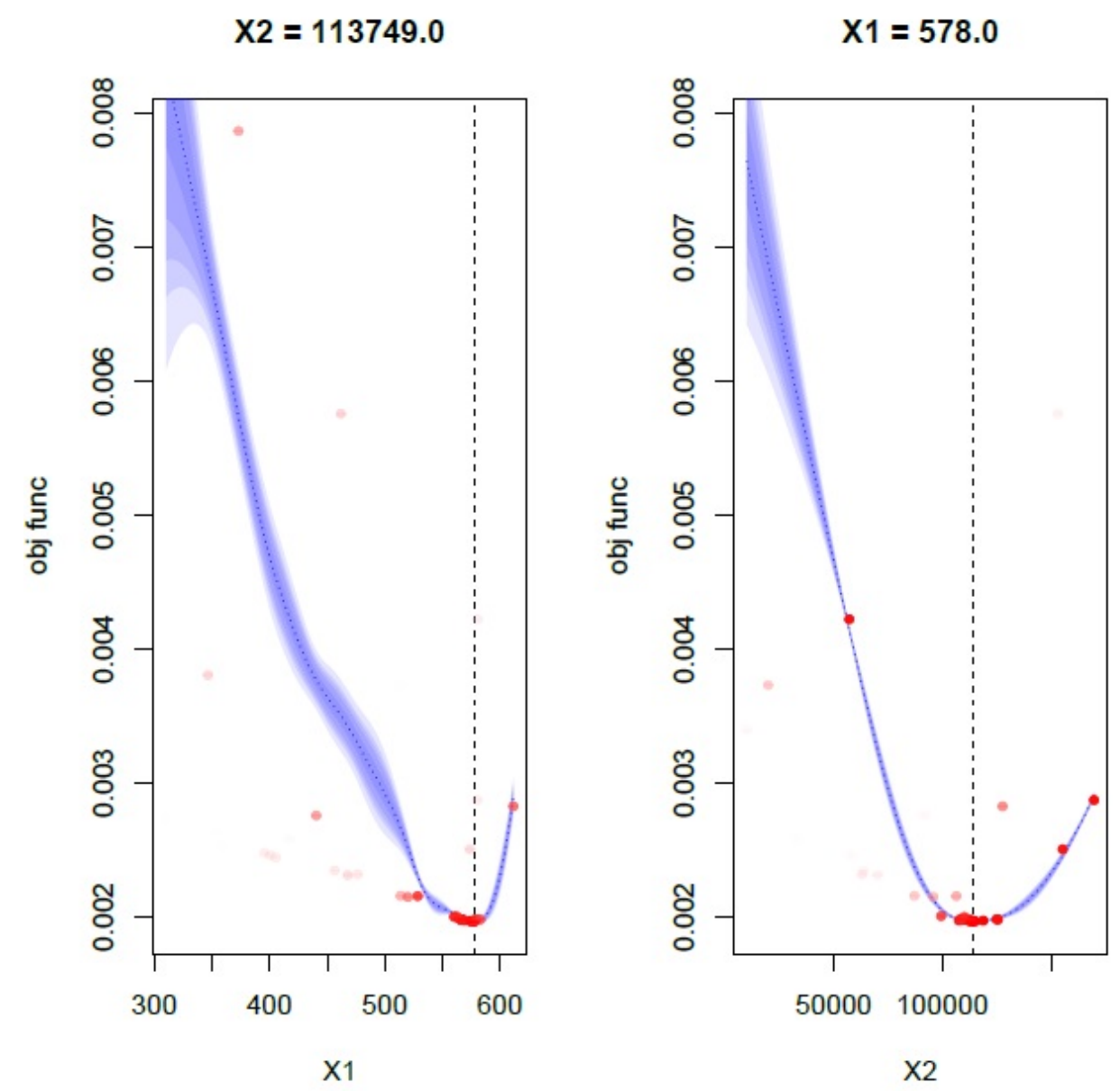

a)

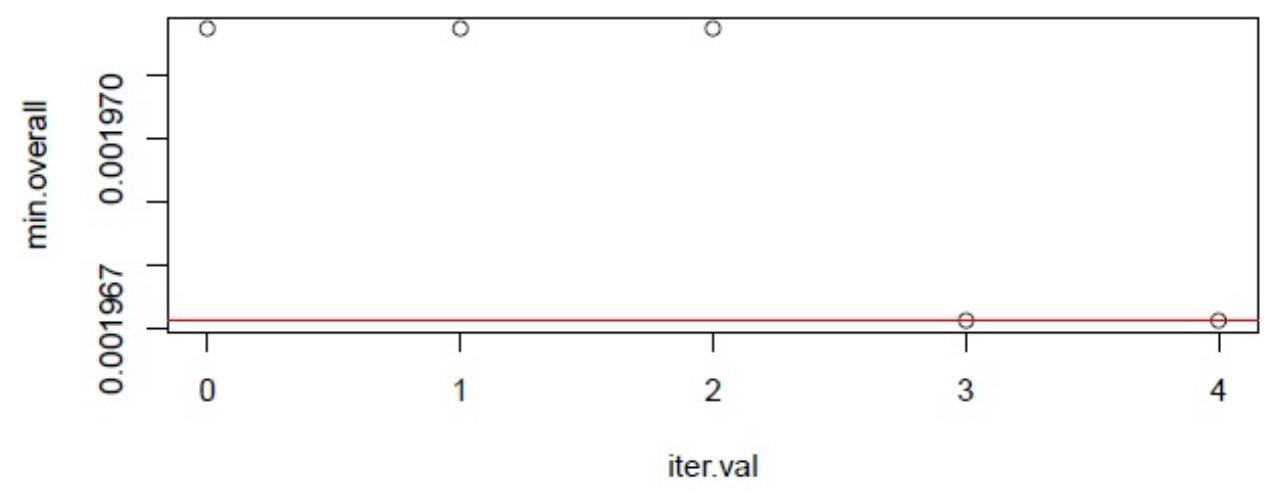

b)

Figure 4.12: Optimized value of DOE temperature. a) Optimized value of DOE temperature and its DOE time with the pbjective function value. b) evolution of objective function during Kriging iterations. 


\section{Predicted Kriging Model, initial+5 iterations}

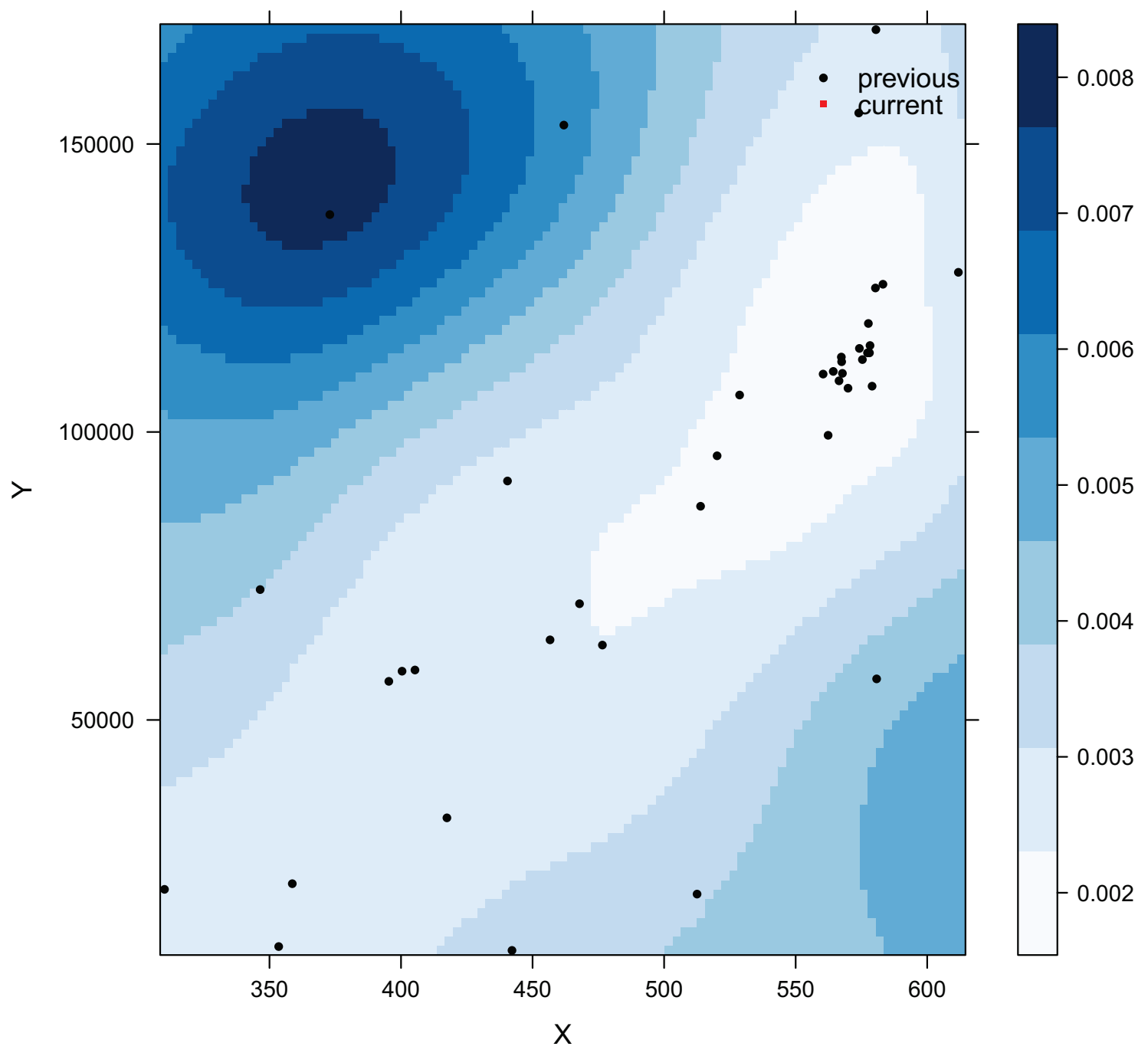

Figure 4.13: 2D plot of objective function EPS as function of DOE temperature, $T_{2}$ and DOE time, $t_{2}$ for the Sutanto start up scenario. 

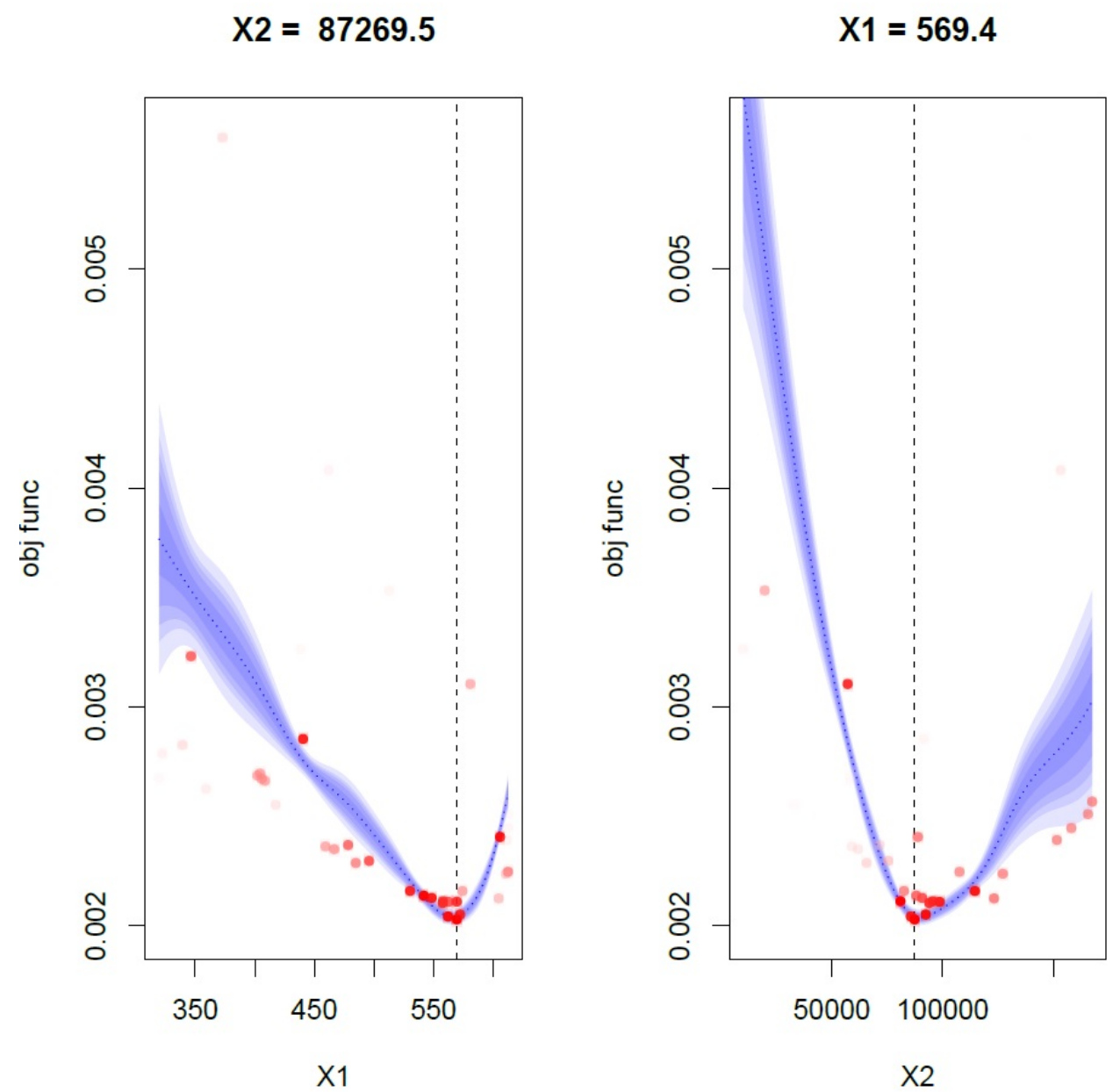

a)

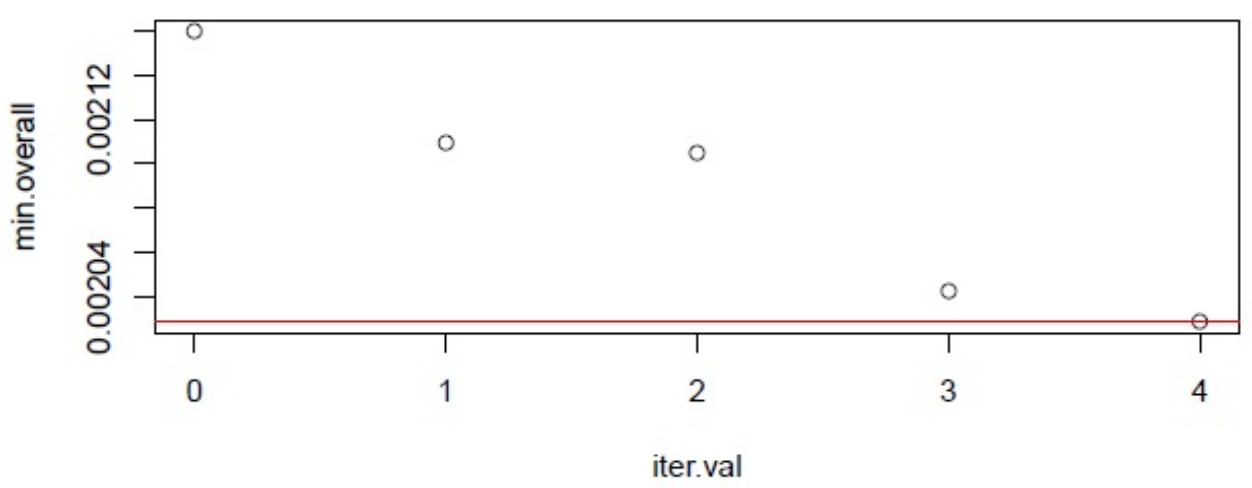

b)

Figure 4.14: Optimized value of DOE temperature. a) Optimized value of DOE temperature and its time with the objective function value. b) evolution of objective function during Kriging iterations. 


\section{Predicted Kriging Model, initial+5 iterations}

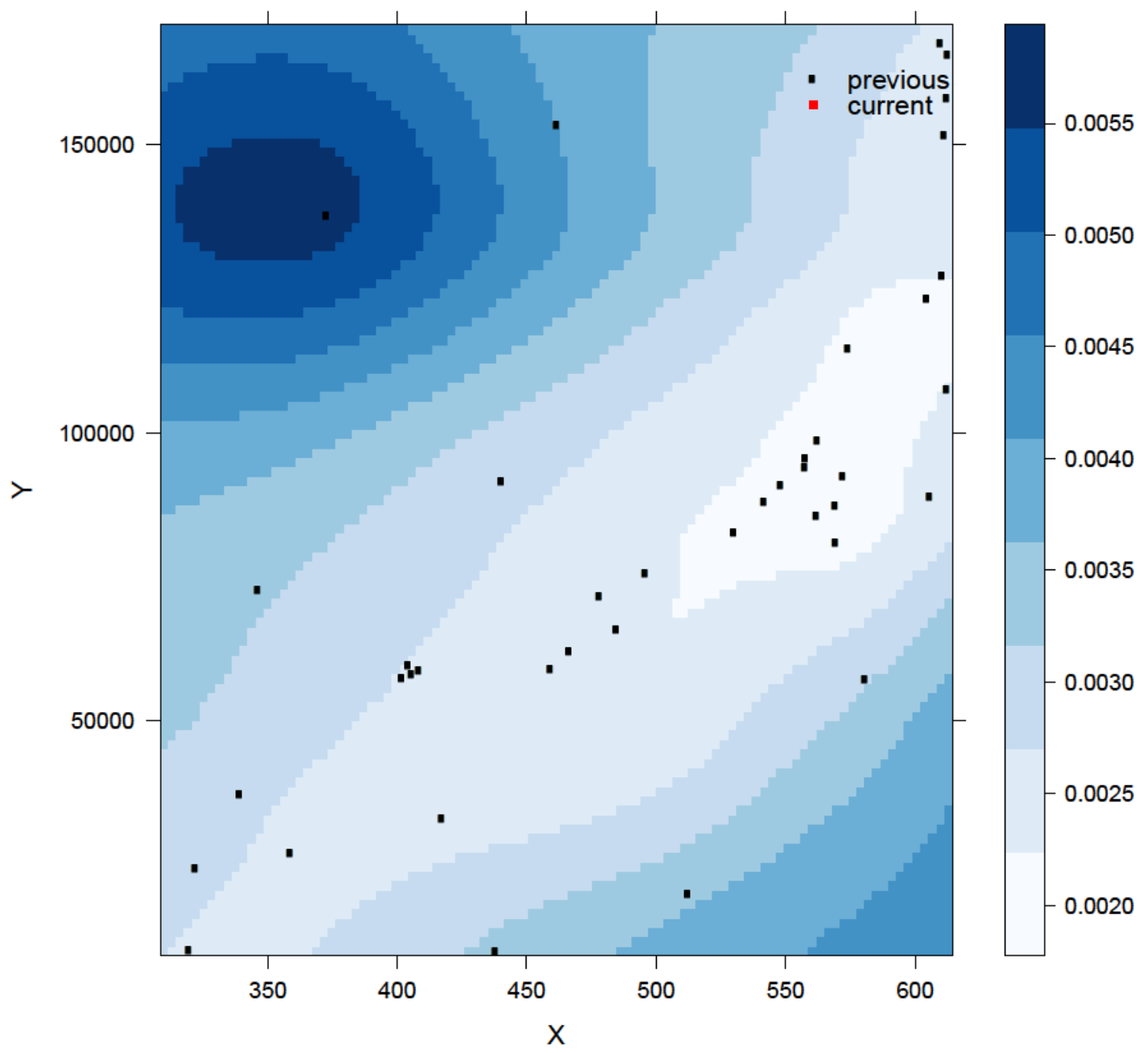

Figure 4.15: 2D plot of objective function EPS as function of DOE temperature, $T_{2}$ and DOE time, $t_{2}$ for the case which the pressure is ramped up linearly from $5 \mathrm{MPa}$ to $25 \mathrm{MPa}$. 

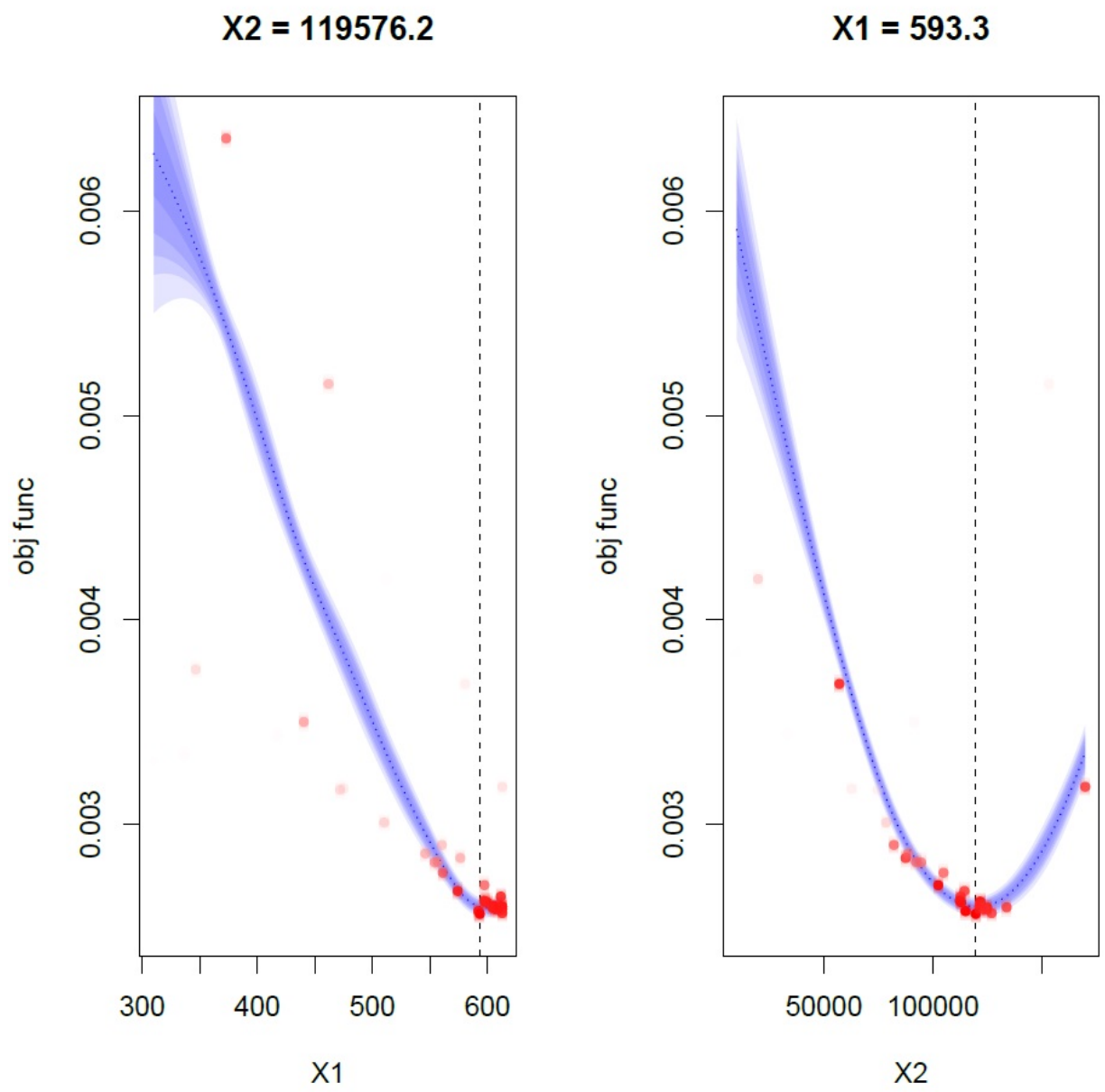

a)

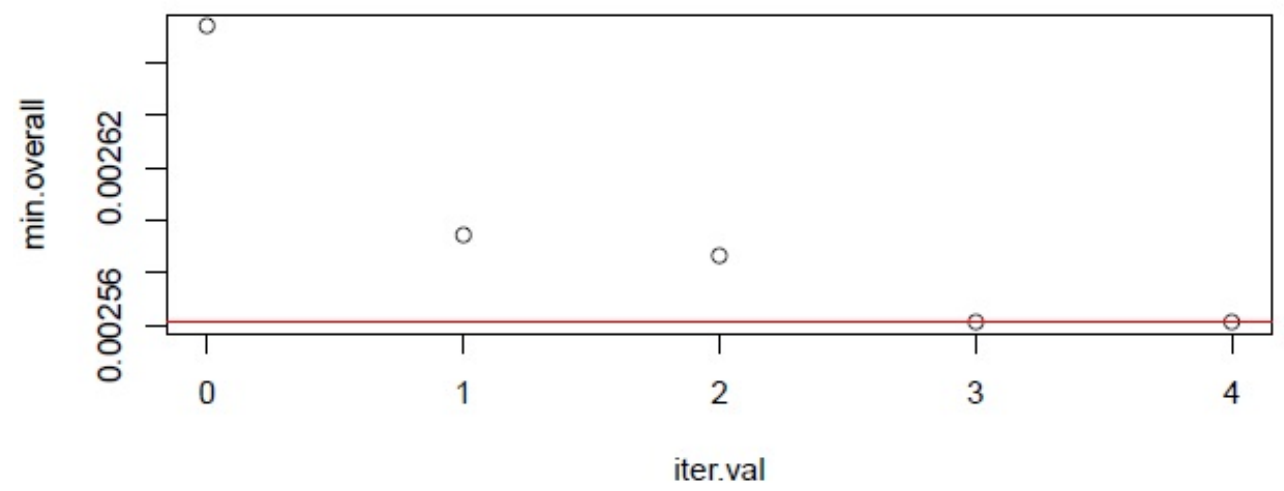

b)

Figure 4.16: Optimized value of DOE temperature. a) Optimized value of DOE temperature and its DOE time with the objective function value. b) evolution of objective function during Kriging iterations. 


\section{Predicted Kriging Model, initial+5 iterations}

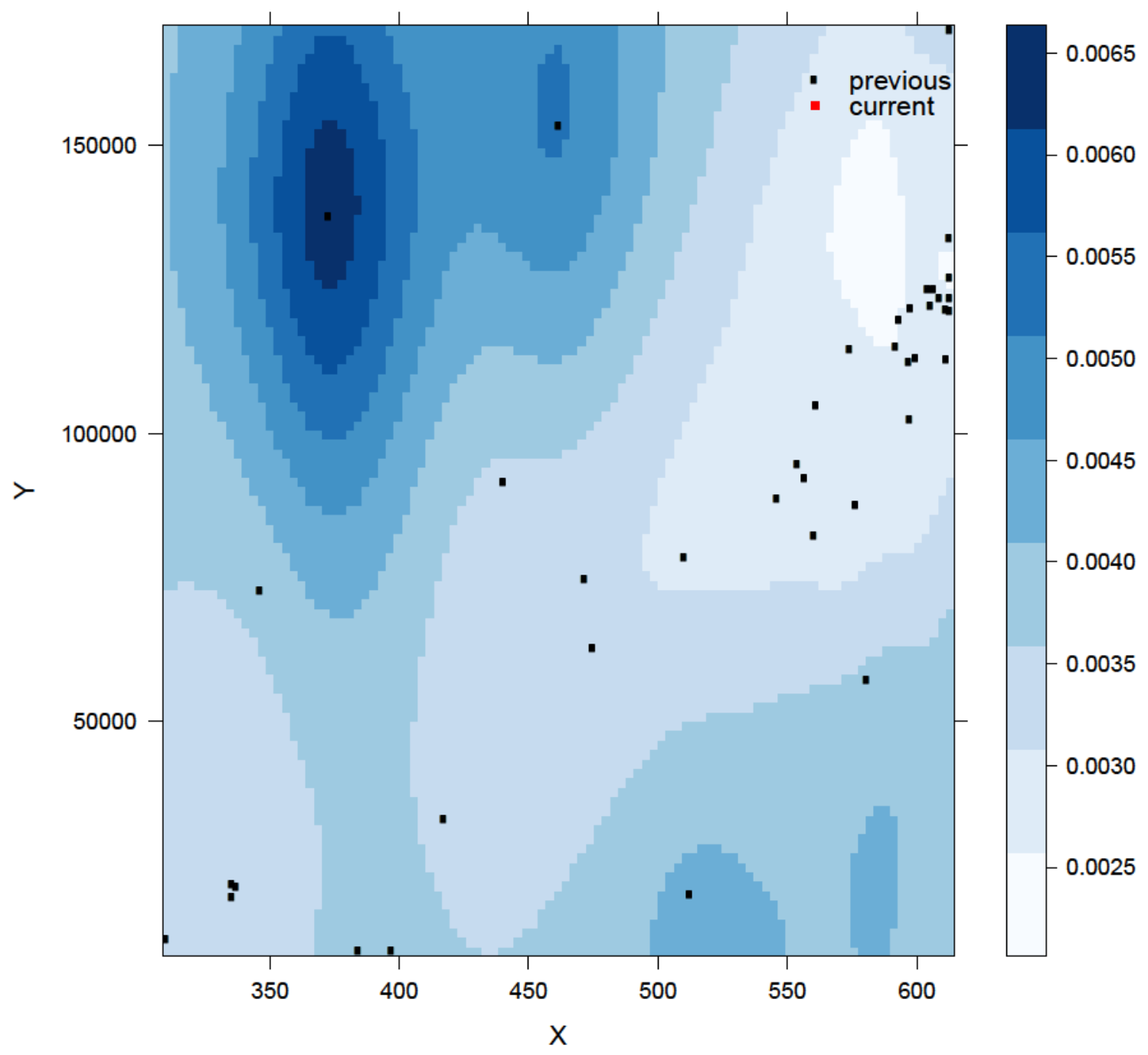

Figure 4.17: 2D plot of objective function EPS as function of DOE temperature, $T_{2}$ and $\mathrm{DOE}$ time, $t_{2}$ for the case which the pressure is ramped up linearly from $10 \mathrm{KPa}$ to $25 \mathrm{MPa}$. 


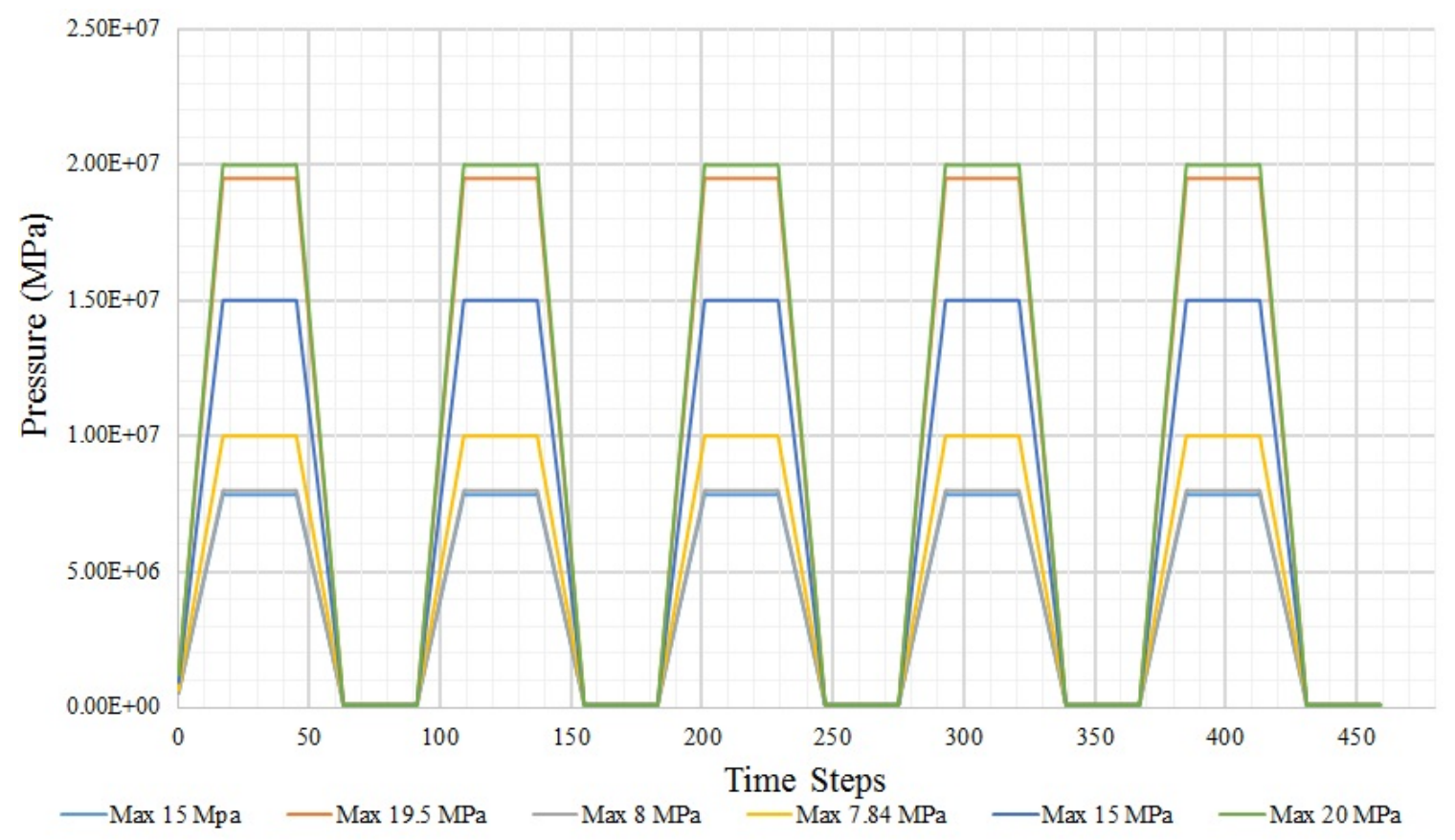

Figure 4.18: Pressure cycles used to find shake down bounds.

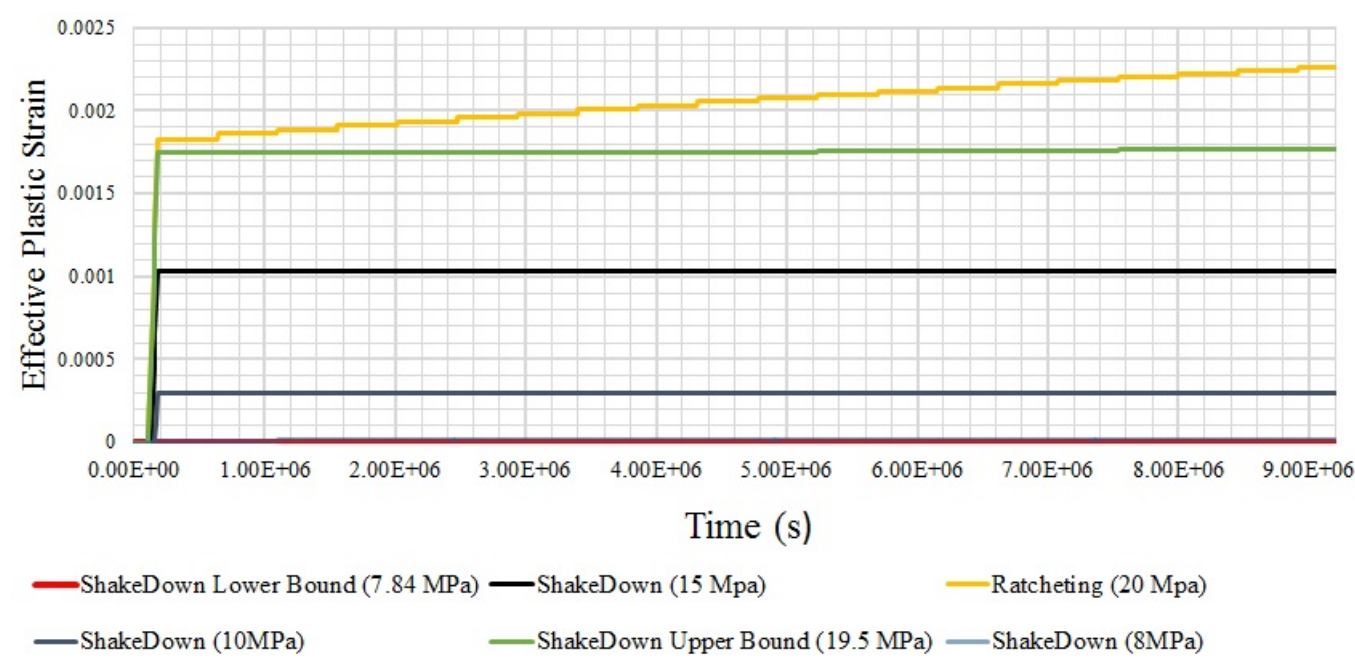

Figure 4.19: The upper and lower bounds of shakedown with comparing of the EPS for each loading case that is shown in Fig. 4.18 for point1. 


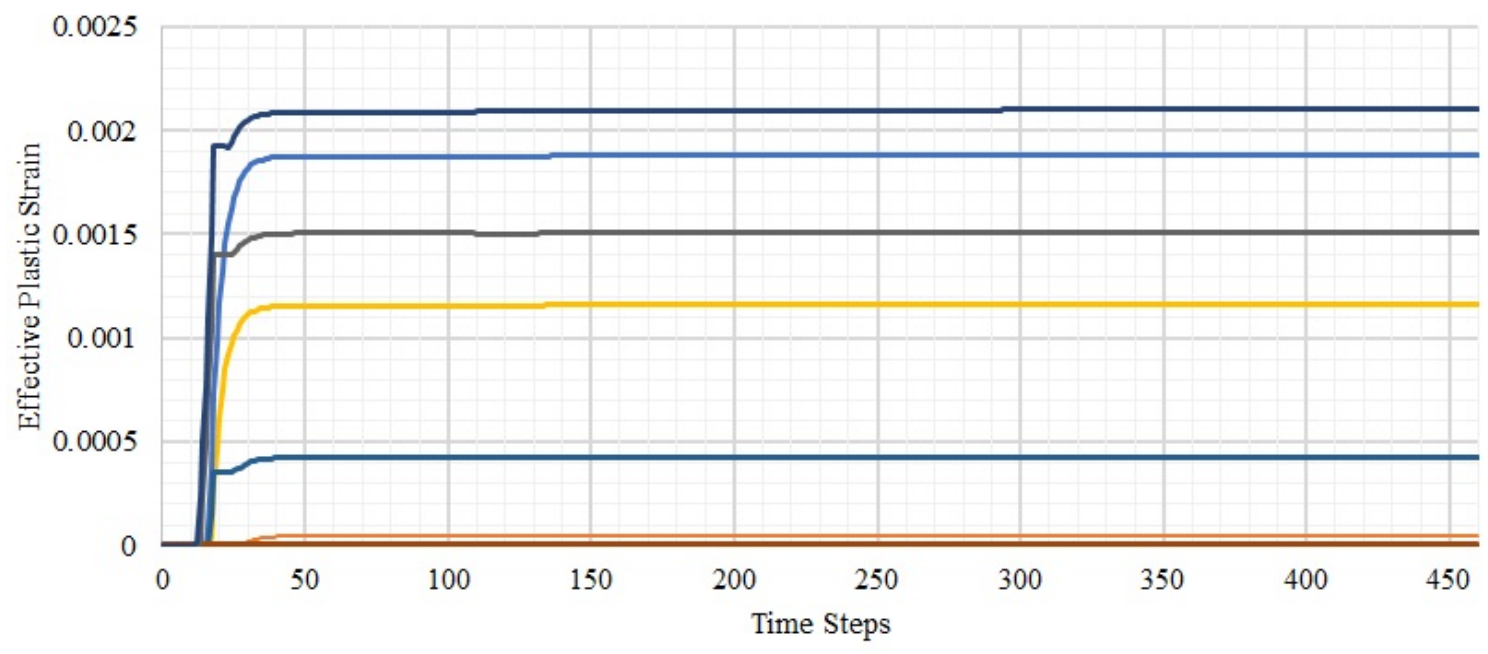

- Point 3 , Lower shakeDown bound at $10 \mathrm{MPa}$

Point 3 , at $24 \mathrm{MPa}$

- Point 2, at $17 \mathrm{MPa}$

- Point 2 , at $23 \mathrm{MPa}$

- Point 3 , at $17 \mathrm{MPa}$

Point 3 , at $25 \mathrm{MPa}$

Point 2 , Lower shakeDown bound at $15.05 \mathrm{MPa}$

- Point 2 , at $25 \mathrm{MPa}$

Figure 4.20: The upper and lower bounds of shakedown with comparison of the EPS for points 2 and 3 . 


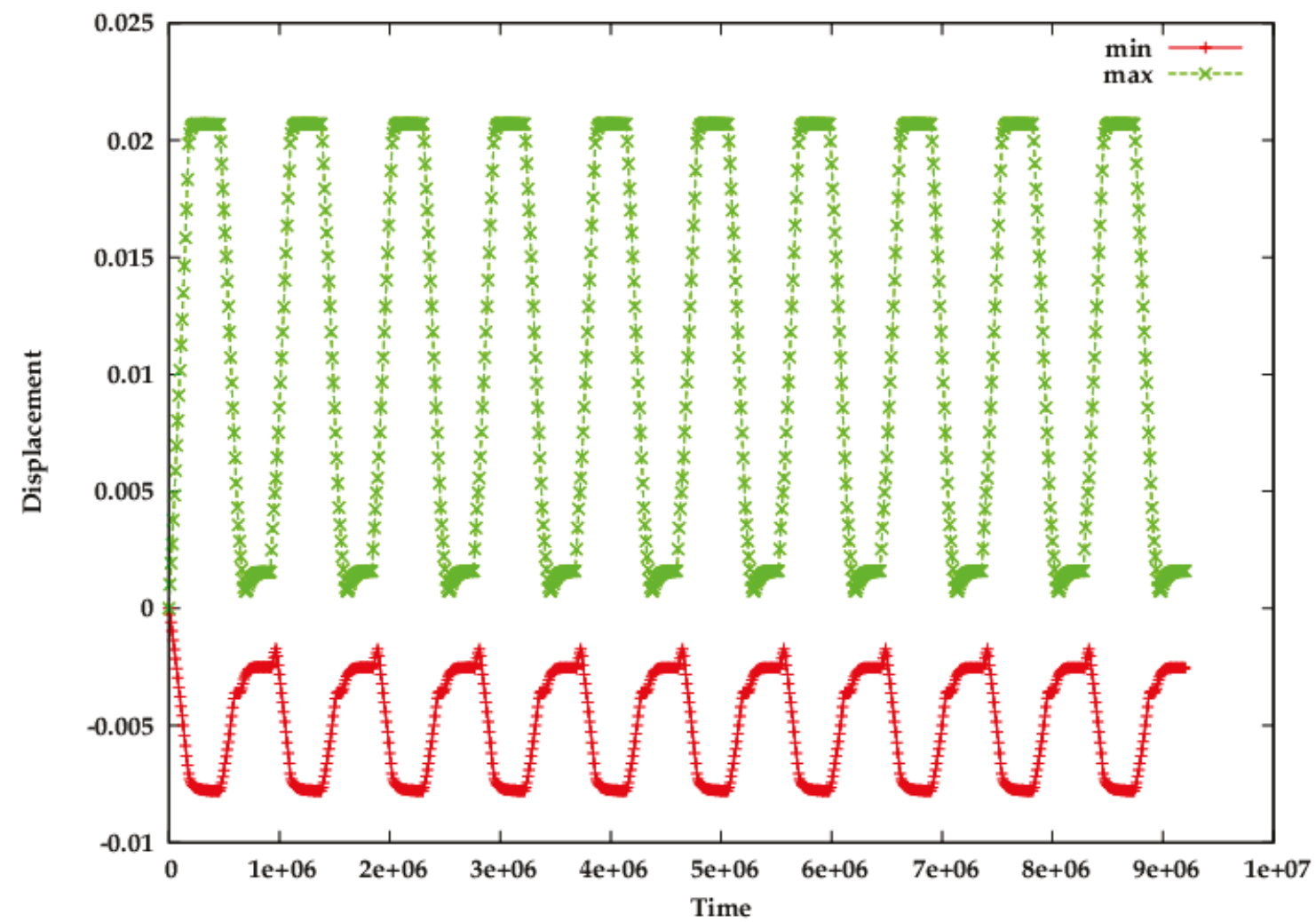

Figure 4.21: Displacement versus time for point 1 in the domain which is shown to be increasing. 


\section{Chapter 5}

\section{Optimization Of GEN IV plenum}

\section{geometry}

\section{$5.1 \quad$ Introduction}

As it was discussed in chapter 1, the use of design by analysis (DBA) is becoming common in ASME codes [27]. There are comprehensive papers that investigate the design and optimization of mechanical parts such as: turbine blades [28] and nozzles in pressure vessels [29]. Martin et al. [30] tried to optimize one nozzle in a vessel. They ran a full factorial design study (with the total number of 243 analysis at 3 levels) that consisted of 5 independent design variables on a $90^{\circ}$ segment of a vessel. They assumed two load cases for the optimization study: the strength of the material and shake down of the domain. The sensitivity of the objective function to design parameters was investigated by fitting polynomials to the design parameters. They concluded that among five parameters, three of them have a main effect on the 
analysis.

\subsection{Problem definition}

To facilitate optimization of design of the plenum, there is a need to have a parametric design including a parametric mesh for each part. A parametric mesh has three important advantages. The parameters can be used as design variables to optimize the design using Design of Experiment and optimization. Because a parametric mesh can be created automatically by a computer program, the user does not need to create new meshes to explore a design space. Because the parametric meshes are created automatically, time is minimized and the risk of human error is reduced.The designer should consider, the interaction of parts in the splitting the domain into different parts. Parametrization will ease the procedure of choosing design parameters in the optimization of the plenum. Each parametrized part has a specified number of parameters. There is always a trade off between minimizing the number of parameters and defining the topology of a domain properly. In other words, partitioning the domain will be done in such a way that it minimizes the number of parameters for parameterization.

Up to now, parametric meshers have been completed for three part of the GEN IV plenum. Each parametrized mesh has some parameters to control the geometry of the part and some parameters that defines the mesh. The parametric mesher for each of these three part is described below: 


\subsubsection{Tube sheet}

The tube sheet at the bottom of the plenum contains 336 pressure tubes. It's remarkable to say that, in the new design, the tube sheet is parameterized in a such a way that the number of pressure tubes and the FEM mesh of the tube sheet is a function of three scalar variables; the pitch size, pressure tube diameter and the outer diameter of tube sheet. To achieve the number of pressure tubes that are divisible by 12 , two flags have been added. The additional flags make it possible to put an additional pressure tube at $45^{\circ}$ if it is needed to make the total number of pressure tubes divisible by 12 and to shrink the distance between the outer circle and the nearest pressure tube (Fig 5.1 a))

Each hole contains a pressure tube. Associated with each hole in the tube sheet will be a sub-domain that will be solved for its Schur complement. For each subdomain thermal and stress will be solved separately and the results will be reported to make the total stiffness matrix. Solving the tube sheet and each pressure tube with their Schur complement will decrease the solving time (See Fig 5.1 b)).

\subsubsection{Plenum}

The plenum is parametrized by 11 parameters (See the Fig 5.2.). The parametrized mesher for the plenum could be improved. For example; the center of the inner and outer fillet should not be the same. It seems that, joining the fillet to the cylinder body should be redesigned with a slope to reduce the stress concentration. 


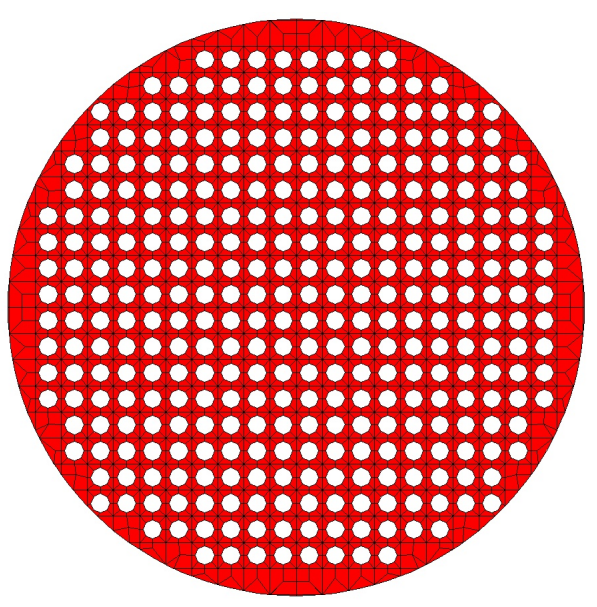

a)

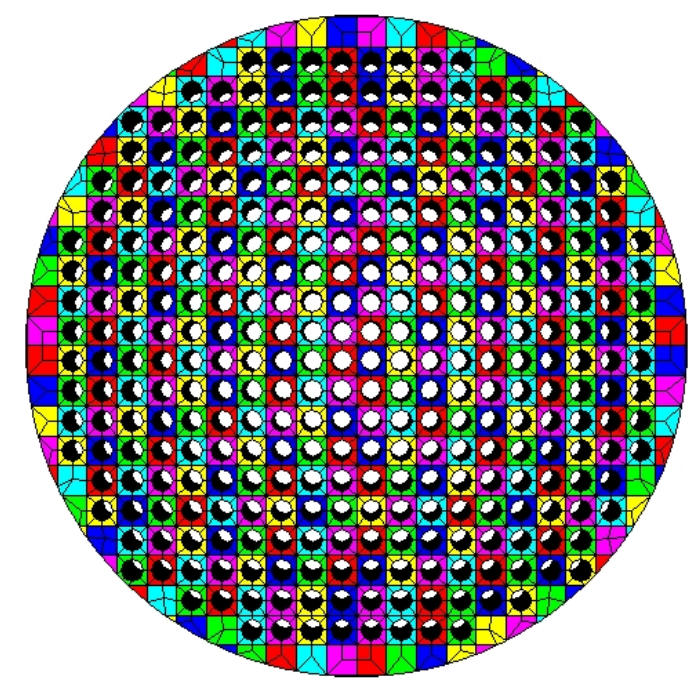

b)

Figure 5.1: Parametrized tube sheet mesher; a) 2D view b) Sub-domains of tube sheet.

\subsubsection{Nozzle and Vessel mesher}

The parametric 3D nozzle meshers, which are coded in VrSuite, contain 10 different parameters. These 10 parameters could be sorted into three different sets: 1) number of elements in each section; 2) the connection between the nozzle and the vessel with base size that defines the boundary of the nozzle mesh with the vessel; 3) the size of different parts inside the base. Due to the complexity of the parts, there is always a possibility of intersecting the parts in a way that will destroy the main topology of the nozzle. All parameters of the nozzle, have been classified as shown below (see Figure 5.3):

\section{Inner parameters are:}

1-Nozzle buffer size

2-Outer radius of nozzle 
3-Thickness of nozzle

4-Height nozzle

5-Height 1 connection

6-Height 2 connection

7-Thickness connection (the last three parameters defines the fillet of nozzle).

\section{Defining grid size in the total nozzle:}

1-Number of elements in the first connection

2-Number of elements in the second connection

\subsubsection{Constraints}

Based on trial and error, some constraints were defined. All parameters were kept constant except one and by testing visually the proposed constraints have been created. More tests are needed to be confident that all of these constraints are necessary and sufficient:

buffer size $\leq 1 / 5^{*}$ base width or length

(outer radius theight 2 connection) $/ 4 \leq$ base square

outer radius of nozzle $>$ thickness nozzle

height nozzle $\geq 1.5$ height 1 connection

heigh1 connection $\leq 2^{*}$ height 1 connection

thickness of nozzle $>2^{*}$ height1 connection

The above list of constraints are not necessarily the final constraints. There is still a possibility to add more constraints. 
To attach the nozzles to the vessel, the vessel should have the capability of detecting the buffer size of the nozzle and fit it to the vessel mesher. The vessel parameters which controls the interaction of vessel and nozzles are:

\section{Vessel parameters include:}

1-Outer radius of base

2-Thickness Base

3-Base Length

4 -Vessel $d X$

5-Number of elements in thickness and nozzle height

To replicate each nozzle, the number of nozzles and the angle of each nozzle were defined. So the tolerance of putting nozzle in its exact position is $d x / 2$. To reduce the tolerance $d x$ should be smaller which is applicable to the point that the number of elements doesn't exceed the limit that computer run out of memory.

To balance the force, two flags were added that make caps at the end of cylinder and the nozzles. Fig. 5.4 shows the vessel mesh most similar to the AECL design that is meshed by the parametric vessel mesher.

\subsection{The sensitivity of vessel mesher to its param- eters}

To find the sensitivity of the objective function to design parameters of the vessel mesher to the parameters which made it, thermal and stress analysis have been done. The boundary conditions are the same as in analysis that is done on the reference 
design. EPS is set as objective function. The analysis is done as a multi-project to see the effect of changing the value of each parameter between its upper and lower bounds on the EPS. The highest value of EPS are at the inner edge of nozzles. The topology of the mesh should be changed to avoid this stress concentration. Figure 5.5 depicts the maximum EPS for one complete start-up shut down cycle.

\subsubsection{Effect of changing nozzle angle and nozzle height on EPS}

The vessel with outlet nozzles but without inlet nozzles has been used as domain. The angle of the nozzle is changing from $20^{\circ}$ to $70^{\circ}$ from the $\mathrm{x}$ axis in the xy plane. The other nozzles in other quadrants have the same pattern. The second nozzle is symmetric to the yz plane and third and fourth nozzle are mirror images of the first and the second nozzle to the the xz plane. The result shows that (Fig. 5.6) at $45^{\circ}$ the EPS is maximum and as it approaches to zero or $90^{\circ}$, the EPS decreases. This means that as the nozzle get closer to each other they make each other stronger.

The same analysis was done to find the effect of the height of the nozzle on the maximum EPS. The height of outlet nozzles was varied from the bottom caps to the upper caps. The first nozzle is located at $45^{\circ}$ from $\mathrm{x}$ direction and all nozzles are orthogonal to each other. As the nozzles get close to the caps the EPS decreases. This shows that as nozzles approach to the end hemisphere they will strengthen the nozzles (Fig. 5.7).

The uncertainty is high in the analysis because the mesh is coarse and fitting the polynomial function to the changes has a problem because the change of the mesh 
function is not smooth enough;i.e, the parametric mesher can only change the nozzle angle by increments controlled by FEM element size. 


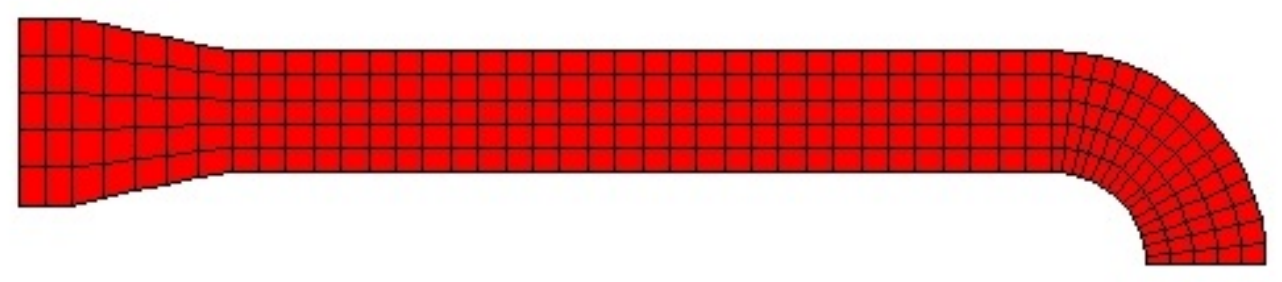

Figure 5.2: Parametrized Plenum.

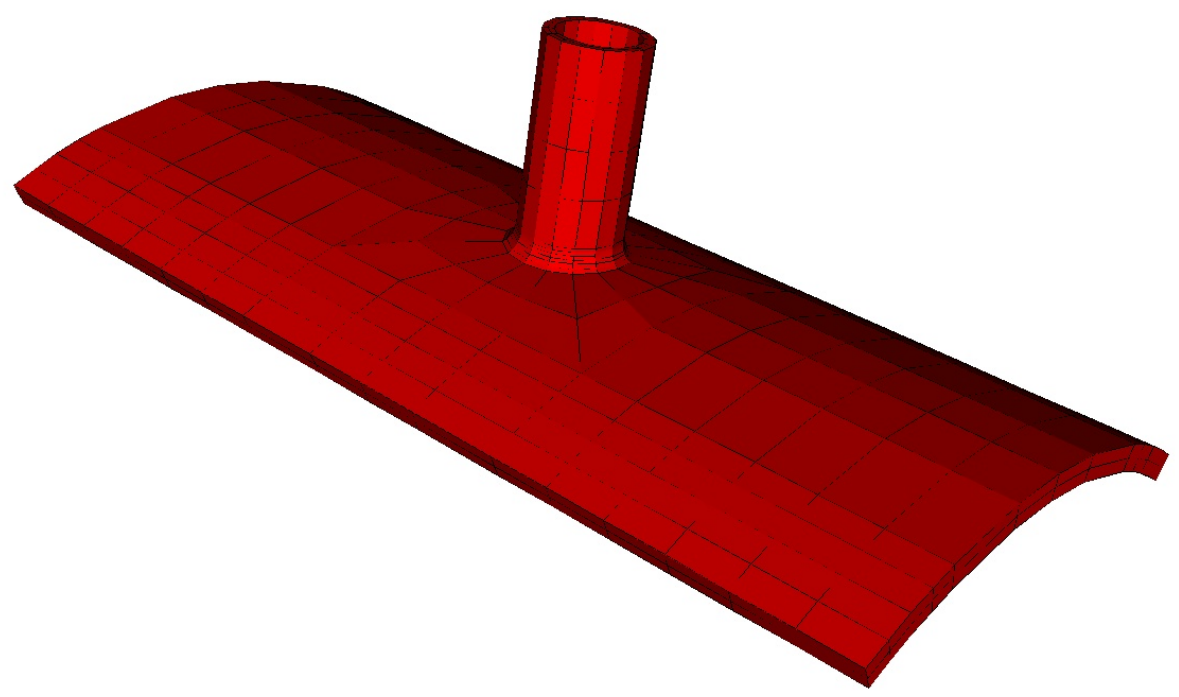

Figure 5.3: parametric FEM mesh generated with 3 separate parts. 


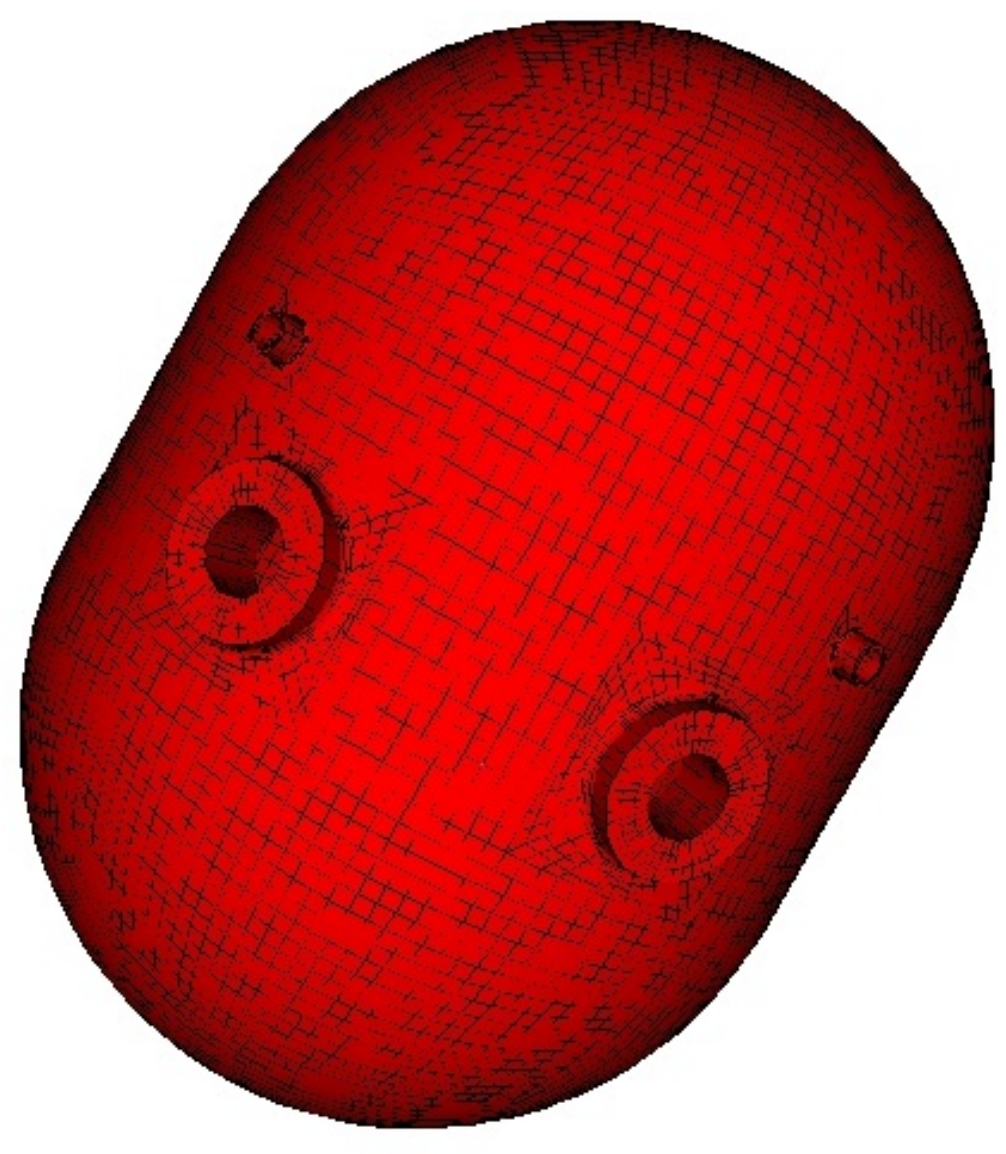

Figure 5.4: The meshed vessel with vessel that is most similar to the mesher to the AECL design . 


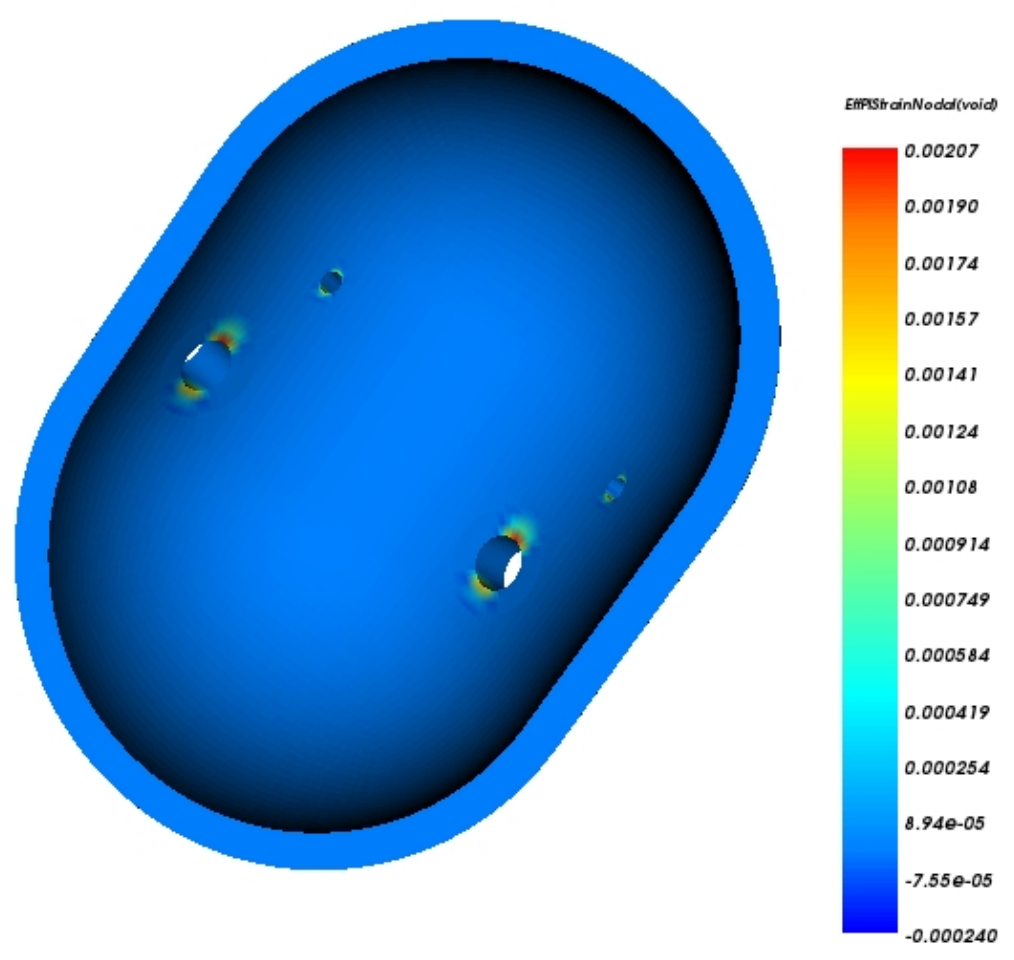

Figure 5.5: The maximum EPS for vessel that is similar to AECL geometry that is meshed with vessel mesher. 


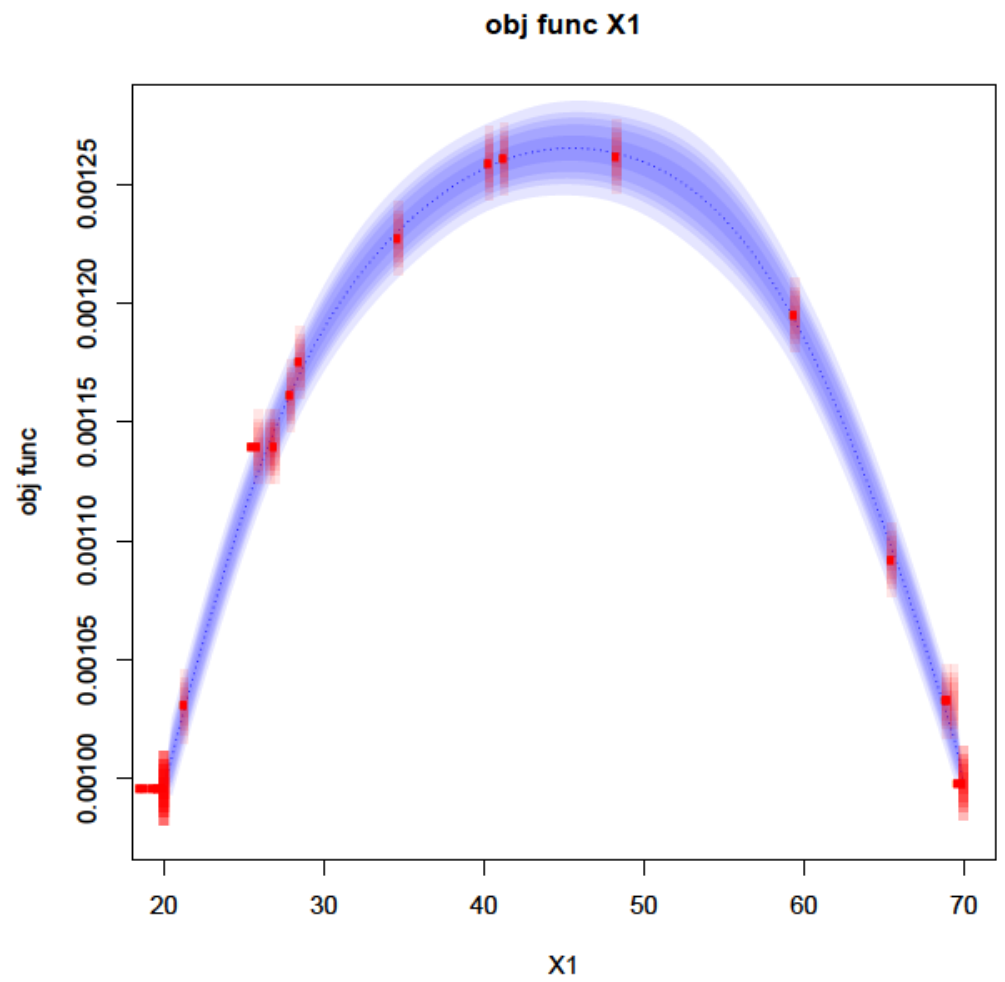

Figure 5.6: The maximum EPS as a function of the angle between nozzles. 


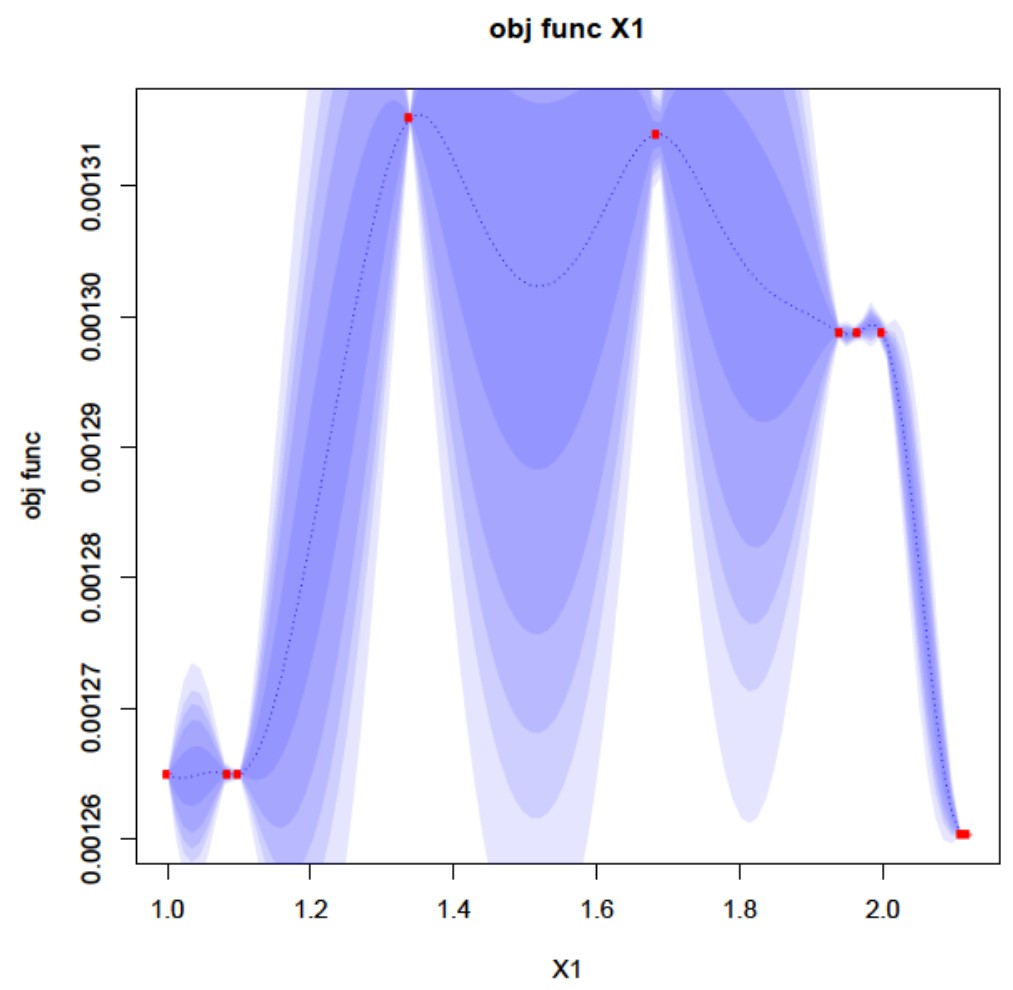

Figure 5.7: The maximum EPS change as a function of the height of nozzles. 


\section{Chapter 6}

\section{Conclusion and possible Future}

works

\subsection{Bree test verification with VrSuite}

Although there were some discrepancies with implementation of 1D Bree test with 3D axisymmetric analysis, the shake down behavior of a cylinder sheath was successfully

achieved. The importance of getting the behavior of Bree digram proved that, VrSuite can capture the shake down, ratcheting and reverse plasticity of the plenum. This enables the designer to optimize the design for shake down of the structure as objective function after finite number of cycles. 


\subsection{Reference analysis conclusion}

This thesis successfully demonstrated the capability to analyze a 3D transient nonlinear coupled holistic macroscopic thermo-viscoelastic-plastic model of the GEN IV

plenum. This approach solved a stress problem coupled with the temperature field and internal pressure. It also computed EPS, regular and principal nodal stress/strain which identified the areas that have the most elastic or plastic deformation. After completing one startup shut down cycle, it was determined that the design of the top head of the plenum and outer fillets of the outlet nozzles should be improved. Also high stresses are predicted at the sharp corner of the inner surface of the nozzles. It is recommended to round these corners to reduce stresses.

Ten similar cycles of startup, shut-down pressures and temperatures were applied on the plenum. Two points are identified that will ratchet (top of the plenum and hot nozzles) and lead to unelectable plastic strain. Also, there are some other points that experience high EPS: the points that join the vessel to pressure tubes and the corner of the top head, but they are expected to shake down over repeated startup/shutdown cycles. Analysis on the reference design provides the estimated time required for the plenum to reach steady state for any startup shut down cycle.

\subsection{Optimization of startup and shut down applied pressure and temperature profiles}

However, doing analysis on the reference design is preferred, doing optimization to find the optimum startup-shut down pressure/thermal scenarios will take time and 
practically it's not feasible because of long solving time for each iteration of optimization process. The 2D axisymmetric mesh provided by VrSuite to enable analyzing the axisymmetric 3D parts with axisymmetric 2D domains. Also, the Kriging tool in the VrSuite is used to perform the optimization process.

The Sutanto [21] startup scenario was chosen as a reference startup scenario, in which the pressure was a function of saturation temperature. The pressure and temperature profiles were expressed by two piece-wise linear functions defined by three points. The first and the last points were fixed at the upper and lower bounds and the two coordinates time, temperature of middle point was chosen to be the design variables to be varied to minimize the EPS. The optimum value was compared for cases that both temperature and pressure were ramped up linearly. It was concluded in which the optimum that was found has higher value of EPS in comparison with the first case. Also the effect of changing the lower bound of pressure was investigated. It was observed that, changing the pressure lower bound will increase the value of optimized EPS.

\subsection{Parametrization and future work}

To optimize the design of the GEN IV plenum, the mesh of the plenum should be parametrized. The $2 \mathrm{D}$ plenum, tube sheet and the plenum with nozzles were parametrized. The vessel mesher supported in VrSuite enables the designer to put any number of nozzles with different sizes and different locations in the vessel. The vessel mesher has the capability to optimize each nozzle separately or to optimize sets of nozzles. To preserve the topology of the mesh some constraints were modified. 
Also some design parameters of the vessel mesher were changed to find the interaction with the other design parameters. It was found that, as the nozzle approaches the end caps of the vessel, nozzles became stiffer. The same behavior was observed when the nozzles get close to each other.

\subsubsection{Future works}

Some suggestions for future works are:

1. Investigate possible startup-shut down scenarios with new objective functions; e.g., shakedown.

2. Run optimization of startup scenarios on a parametrized mesh.

3. Define a new support for the plenum. Sharp edges should be avoided to minimize stress concentrations.

4. Parametrize the top head of plenum. Also some of the parametrized meshes could be modified; e.g., the vessel mesher could be enhanced to create an improved design for the inside fillet for the nozzles.

5. The tube sheet mesher should be modified to have a chamfer for each pressure tube. The 2D plenum mesher should accept two eccentric curves as the outer and inner surfaces.

6. Find the interactions between parameters in all parametrized meshes.

7. Run a Taghuchi or Box-Behrken [31], [32] sensitivity analysis for the design parameters of the vessel mesher. 
8. Select the parameters of the design with the largest sensitivity to the objective function and optimize the design for those parameters.

9. Creep analysis and low cycle fatigue should be studied.

10. 1D analysis of Bree test should be done to minimize the difference between the analytical and simulated results of Bree test.

11. By adding Creep analysis to VrSuite, the availability of regions $S_{2}$ and $\mathrm{Rp}$ should be investigated. 


\section{Appendix A}

In this appendix the material properties of SA533 that was used in the thermal and stress solver, will be presented separately [33].

Thermal properties: 


\begin{tabular}{|l|l|l|}
\hline \multicolumn{2}{|c|}{ Stress parameters } \\
\hline & Temperature range ${ }^{\circ} \mathrm{K}$ & value \\
\hline Properties & 273 & 40 \\
& 473 & 40 \\
Thermal conductivity $\left(\mathrm{W} / \mathrm{m}^{\circ} \mathrm{K}\right)$ & 673 & 38 \\
& 1123 & 26 \\
& 1740 & 32 \\
& 1773 & 320 \\
\hline & 273 & 400 \\
Specific heat $\left(\mathrm{Kj} / \mathrm{Kg}^{\circ} \mathrm{K}\right)$ & 1023 & 900 \\
& 1073 & 1300 \\
& 1123 & 500 \\
\hline Thermal density $\left(\mathrm{Kg} / \mathrm{m}^{3}\right)$ & 1753 & 600 \\
\hline
\end{tabular}

Table 1: The temperature dependent material properties of SA533 that was used by thermal solver.

\begin{tabular}{|l|l|l|}
\hline \multicolumn{3}{|c|}{ Stress parameters } \\
\hline Properties & Temperature range ${ }^{\circ} \mathrm{K}$ & value \\
\hline \multirow{4}{*}{ Yield stress $(\mathrm{Pa})$} & 273 & $3.4 \mathrm{e} 8$ \\
& 673 & $2.6 \mathrm{e} 8$ \\
& 973 & $3 \mathrm{e} 7$ \\
& 1273 & $3 \mathrm{e} 6$ \\
& 1773 & 7000 \\
\hline \multirow{4}{*}{ Hardening $(\mathrm{Pa})$} & 273 & $2 \mathrm{e} 9$ \\
& 673 & $1.8 \mathrm{e} 9$ \\
& 773 & $1 \mathrm{e} 8$ \\
& 1700 & $1 \mathrm{e} 8$ \\
\hline \multirow{3}{*}{ Young modulus $(\mathrm{Pa})$} & 273 & $2.1 \mathrm{e} 11$ \\
& 673 & $1.8 \mathrm{e} 11$ \\
& 773 & $1 \mathrm{e} 10$ \\
& 1700 & $1 \mathrm{e} 5$ \\
\hline \multirow{2}{*}{ Poisson's ratio } & 273 & 0.3 \\
& 673 & 0.3 \\
\hline Viscosity $\left(\mathrm{m}^{2} / \mathrm{s}\right)$ & 1773 & 0.48 \\
\hline
\end{tabular}

Table 2: The temperature dependent material properties of SA533 that was used by stress solver 


\section{Appendix B}

This appendix will present the computation of effective stress, effective plastic strain and external free convection.

\section{Effective stress and effective plastic strain:}

The von Mises yield criterion states that yielding occurs when the effective or von Mises stress reaches the yield stress of the material in uniaxial tension. The effective stress is defined as [34]:

$$
\sigma_{e}=\sqrt{3 J_{2}}
$$

where $J_{2}$ is known as the second invariant of the deviatoric stresses. In terms of principal stresses $J_{2}$ will be:

$$
J_{2}=\frac{1}{6}\left(\left(\sigma_{1}-\sigma_{2}\right)^{2}+\left(\sigma_{2}-\sigma_{3}\right)^{2}+\left(\sigma_{3}-\sigma_{1}\right)^{2}\right)
$$

By substituting of $\epsilon_{p 1}, \epsilon_{p 2}$, and $\epsilon_{p 3}$ (which are three principal plastic strains) for $\sigma_{1}, \sigma_{2}$ and $\sigma_{3}, J_{2}$ will be effective plastic strain.

\section{External Free Convection:}

The average convective heat transfer coefficient of a cylinder is determined by the 
relation:

$$
\overline{h_{c y l}}=\frac{\overline{N u_{D}} \kappa}{D i a}
$$

$N u_{D}$ is the average Nusselt number, $\kappa$ is the thermal conductivity of the fluid $\left[W /\left(m^{\circ} K\right)\right]$, and is the diameter of the cylinder $[m]$. The average Nusselt number for a long horizontal cylinder undergoing free convection is determined using the empirical correlation of Churchill \& Chu [35]:

$$
\overline{N u_{D}}=\left[0.6+\frac{0.387 R a_{D}^{1 / 6}}{\left(1+\left[\frac{0.599}{P r}\right]^{9 / 16}\right)^{8 / 27}}\right]^{2}
$$

Prandtl and Rayleigh numbers for a cylinder, given by:

$$
\begin{gathered}
\operatorname{Pr}=\frac{\nu}{D} \\
R a_{a}=\frac{g \beta\left(T_{s}-T_{a}\right) D^{3}}{D \nu}
\end{gathered}
$$

$\nu$ is the kinematic viscosity $\left[\mathrm{m}^{2} / \mathrm{s}\right], D$ is the thermal diffusivity $[\mathrm{m} 2 / \mathrm{s}], g$ is the acceleration due to gravity $\left[\mathrm{m} / \mathrm{s}^{2}\right], \beta$ is the thermal expansion coefficient, $T_{s}$ is the surface temperature and $T_{a}$ is the temperature of ambient air. For the free convection of a vertical cylinder, an alternative Nusselt number correlation is provided by Churchill \& Chu [35].

$$
\overline{N u_{D}}=\left[0.825+\frac{0.387 R a_{D}^{1 / 6}}{\left(1+\left[\frac{0.492}{P r}\right]^{9 / 16}\right)^{8 / 27}}\right]^{2}
$$




\section{Appendix C}

This appendix will compare the exact solution and simulated result for a prescribed convection boundary condition. It also explains the end effect for problem and the effect of choosing long time steps (or a coarse mesh) on the analysis. In the second part, the results of a $2 \mathrm{D}$ and $3 \mathrm{D}$ axisymmetric mesh are compared with the exact solution for both thermal and stress problem.

\section{Problem Description}

The FEM solution of a semi infinite bar with a convection heat transfer and exact solution were compared. The problem is solved for 3 different times. The transient solution for convection heat transfer with evolution of time is analyzed. Also the axisymmetric 2D mesh and 3D axisymmetric and exact solution were verified. In each problem the same cross section was used for 2D analysis, for 3D analysis and for the exact solution.

\section{Domain and Boundary condition}

The rectangular bar with length of $0.45 \mathrm{~m}$ (based on minimum thickness of the plenum) and with the height that satisfies a condition for the element aspect ratio equal to one was considered as domain. The whole bar is divided to equally space

elements (The cross section of each element has an area $0.01 \times 0.01 \mathrm{~m}^{2}$ ). For the 
axisymmetric 3D mesh analysis analysis, the same cross section with different number of circumferential elements was used. All the plots are drawn for $0.45 \mathrm{~m}$ of the total length. The energy equation was solved for thermal problem with convection boundary condition and applied Dirichlet temperature. See the Fig. 1 for contour plots of temperature field.

The convection coefficient assumed $h=1000 \mathrm{~W} / \mathrm{m}^{2 \circ} \mathrm{K}$ and the ambient temperature is considered $T_{\infty}=400^{\circ} \mathrm{K}$. For Dirichlet $\mathrm{BC}$ these values were set: $T_{\text {Dirichlet }, \text { inside }}=300^{\circ} \mathrm{K}$, $T_{\text {Dirichlet }, \text { outside }}=400^{\circ} \mathrm{K}$. The material is SA533.

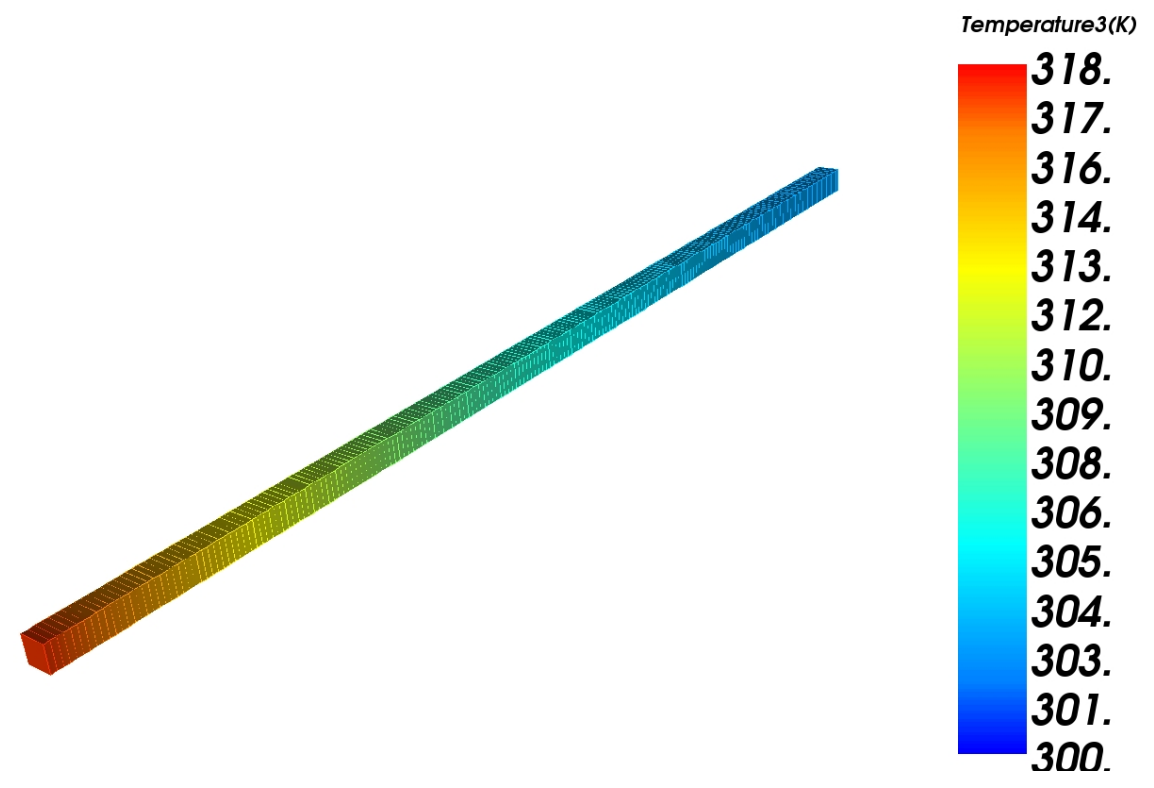

Figure 1: Contour Plots of Temperature Field for Semi infinite Bar.

For the stress problem, $25 \mathrm{MPa}$ inside pressure was applied also the nodal force was computed for 1 rad section of cylinder and was applied to two nodes of the internal edge of a 2D axisymmetric cross section of cylinder (the nodal force will change by changing of the height of an element). To verify the code, the computed 
displacement was applied to the same geometry to internal points and the effective stress was compared with the pressurized cylinder that gave that displacement. The BCs were set as follows:

$$
P_{i}=5 \mathrm{MPa}, P_{o}=0.1 \mathrm{MPa} \text {, Computed Displacement }=2.28817 \mathrm{e}-5 \mathrm{~m} \text {, Nodal force }
$$
for element with height $=0.05 \mathrm{~m}$ equals $62500 \mathrm{~N}$

\section{The exact solution for the problem}

The exact solution for a plate with imposed surface convection is:

$$
\frac{T_{(x, t)}-T_{i}}{T_{\infty}-T_{i}}=\operatorname{Erfc}\left(\frac{x}{2 \sqrt{\alpha t}}\right)-\operatorname{Erfc}\left(\frac{x}{2 \sqrt{\alpha t}}+\frac{h \sqrt{\alpha} t}{\kappa}\right) * \exp \left(\frac{h x}{\kappa}+\frac{h^{2} \alpha t}{\kappa^{2}}\right)
$$

The exact solution for steady state 1D cylindrical problem could be summarized as:

$$
T(r)=\frac{T_{s, i}-T_{s, o}}{\ln \left(r_{i} / r_{o}\right)} \ln \left(\frac{r}{r_{o}}\right)+T_{s, o}
$$

$T(r)\left[{ }^{\circ} \mathrm{K}\right]$ is the radial temperature in each radius of cylinder $r[\mathrm{~m}], T_{s, i}$ and $T_{s, o}$ is the temperature of inside and outside respectively $\left[{ }^{\circ} \mathrm{K}\right], r_{i}$ and $r_{o}$ are inside and outside radius respectively $[m]$.

$$
\begin{gathered}
\sigma_{r}=\frac{\left(p_{1} \cdot r_{1}^{2}-p_{2} \cdot r_{2}^{2}\right)}{\left(r_{2}^{2}-r_{1}^{2}\right)}+\frac{\left(p_{2}-p_{1}\right) \cdot r_{1}^{2} \cdot r_{2}^{2}}{r^{2} \cdot\left(r_{2}^{2}-r_{1}^{2}\right)} \\
\sigma_{t}=\frac{\left(p_{1} \cdot r_{1}^{2}-p_{2} \cdot r_{2}^{2}\right)}{\left(r_{2}^{2}-r_{1}^{2}\right)}-\frac{\left(p_{2}-p_{1}\right) \cdot r_{1}^{2} \cdot r_{2}^{2}}{r^{2} \cdot\left(r_{2}^{2}-r_{1}^{2}\right)} \\
\sigma_{a}=p_{1} \frac{r_{1}^{2}}{\left(r_{2}^{2}-r_{1}^{2}\right)}
\end{gathered}
$$


where $\sigma_{r}$ is radial stress, $\sigma_{t}$ and $\sigma_{a}$ are tangential and axial direction respectively. Where the $P_{1}$ and $P_{2}$ are internal and external pressure [MPa], $r_{1}$ and $r_{2}$ are internal and external radius of the cylinder $[m]$.

\section{Results}

As is obvious in the Figures below, the result of the exact solution is almost identical with the FEM solution for both thermal and stress problems.

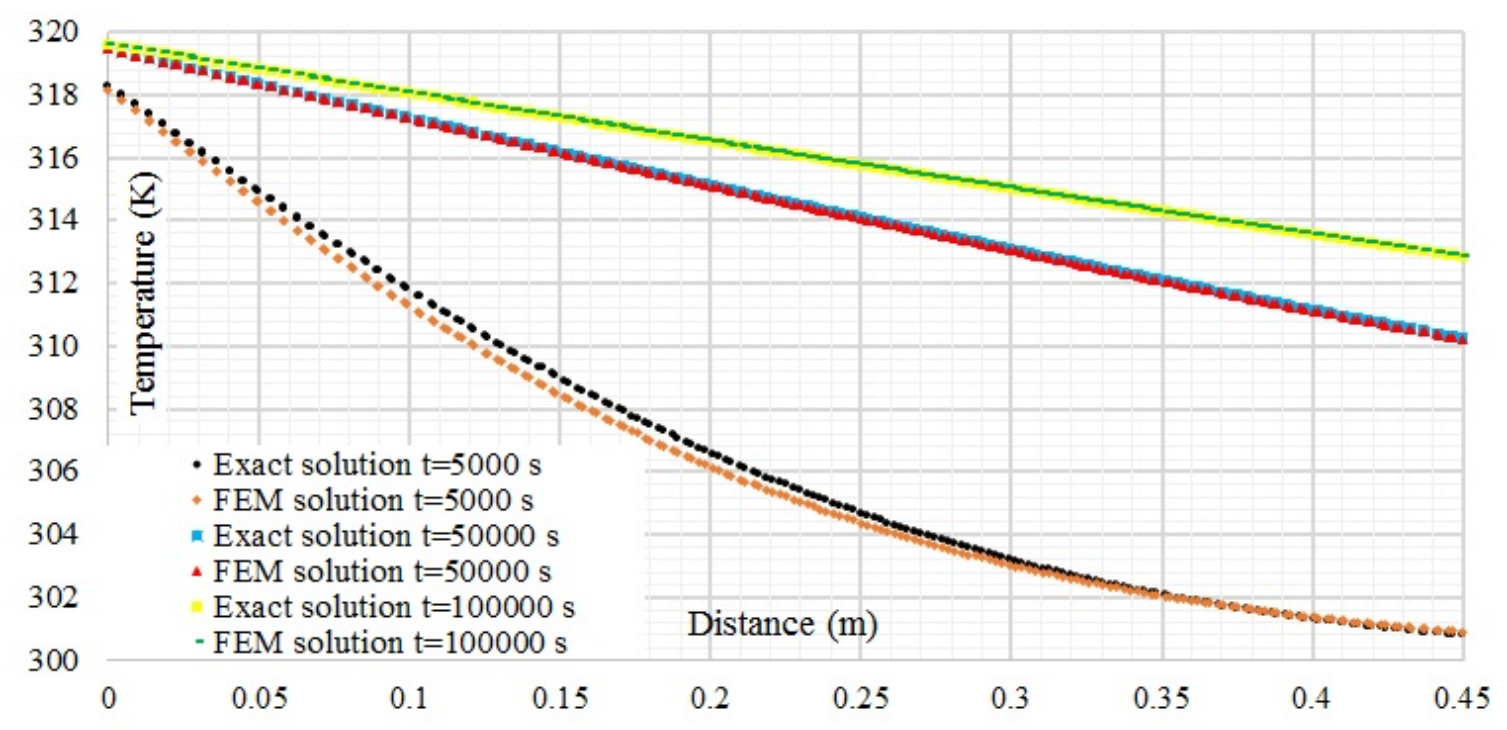

Figure 2: Comparison of FEM solution and exact solution for 3 different times. 


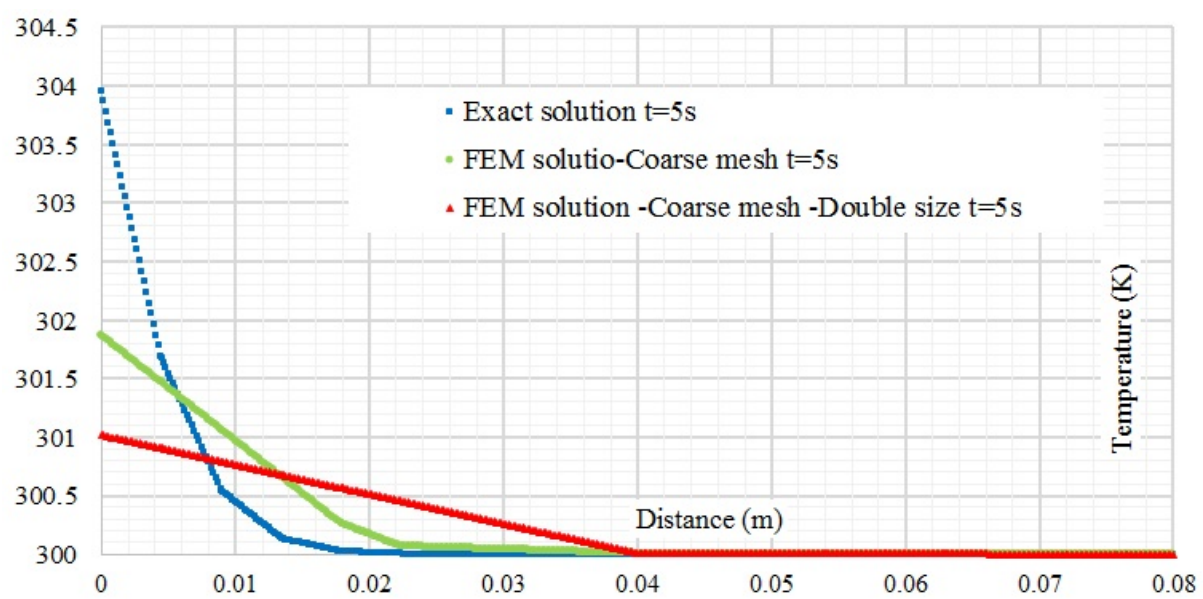

Figure 3: The error which made by choosing wrong time steps or element length.

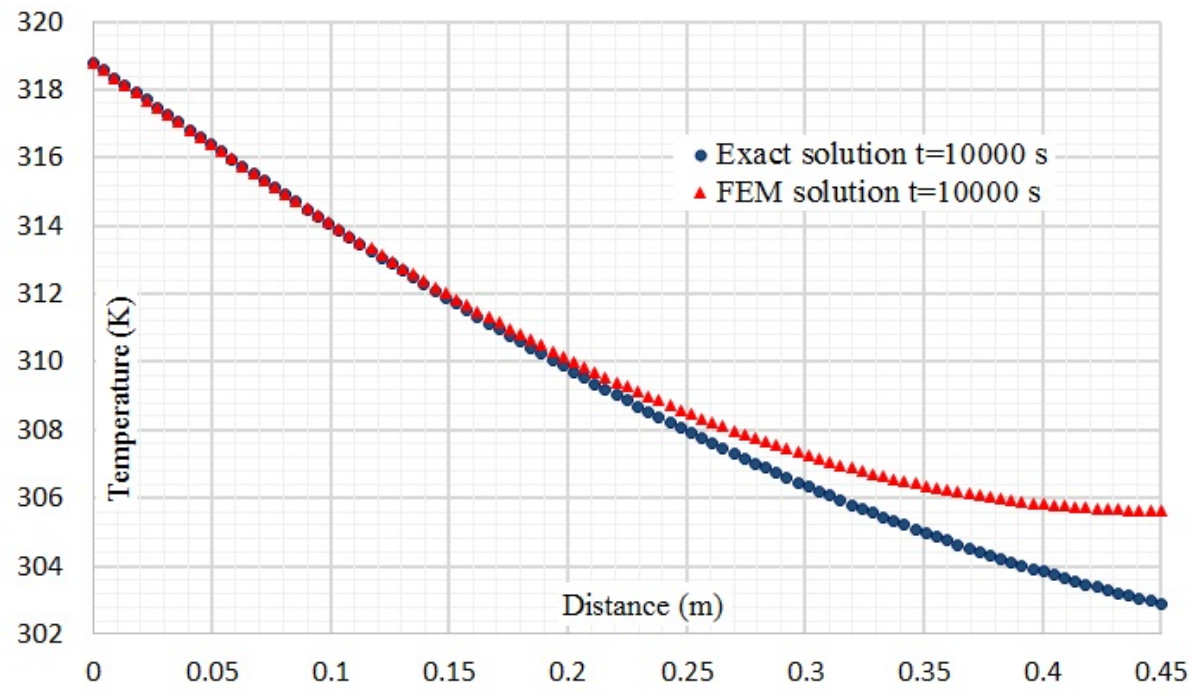

Figure 4: Comparison of FEM solution with end effect and eliminating end effect. 


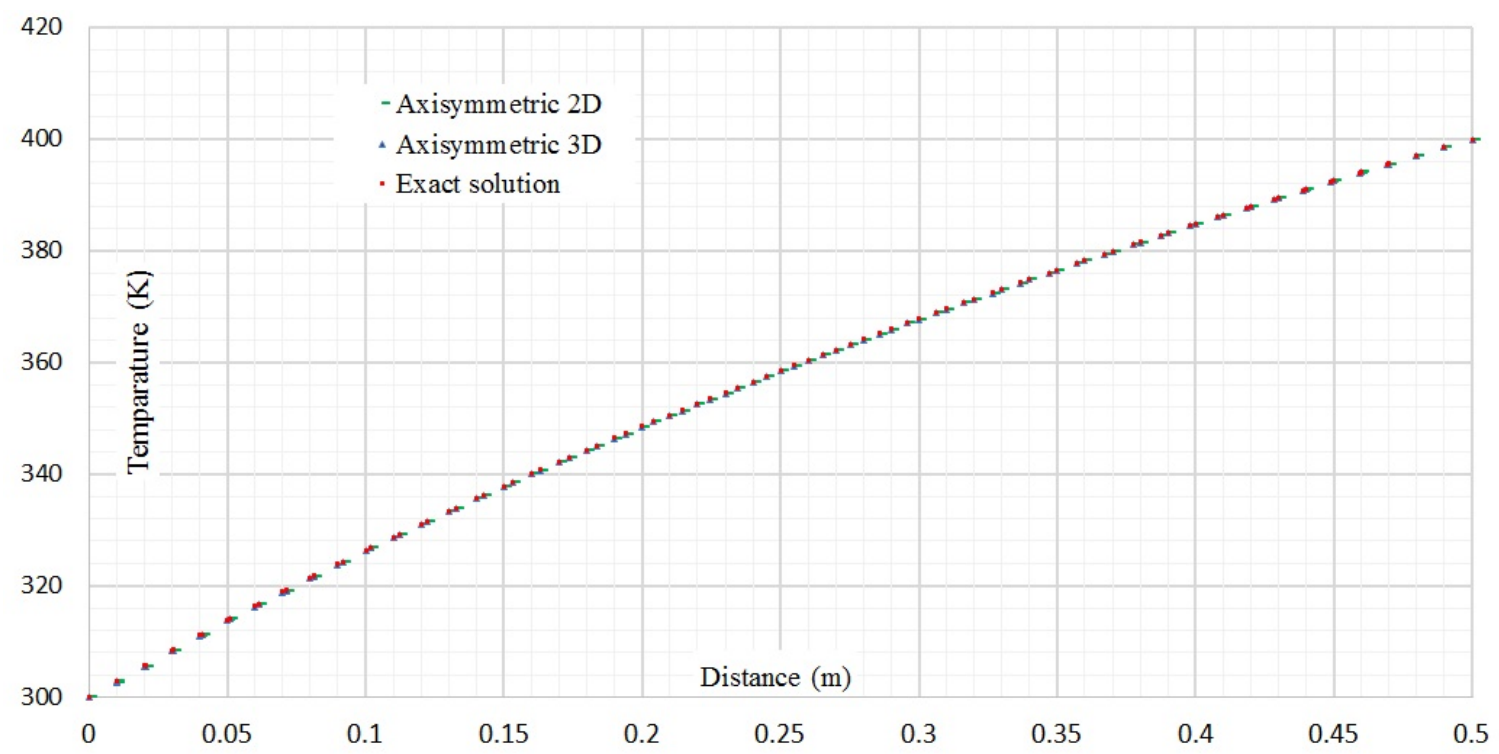

Figure 5: Comparison of temperature field for 2D, 3D and exact solution.

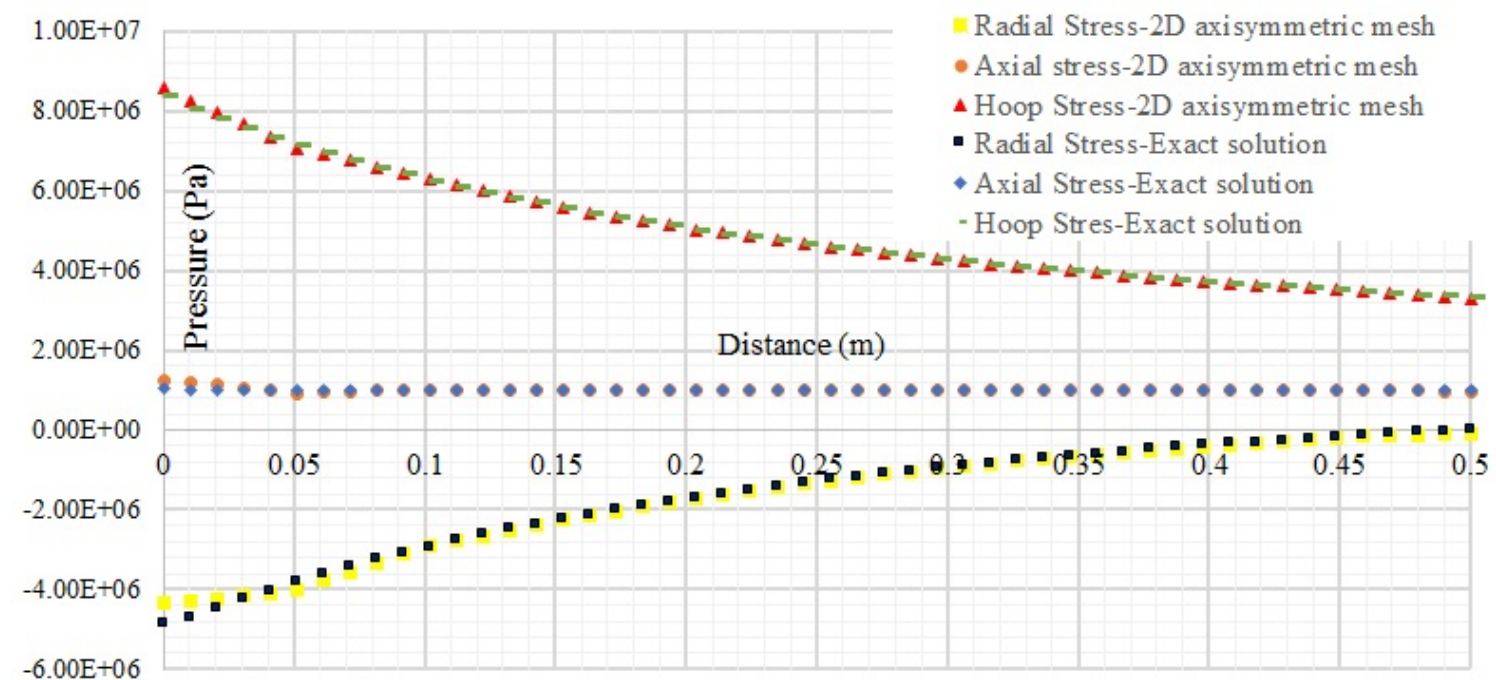

Figure 6: Comparison of nodal stresses for 2D, 3D and exact solution at the last time step. 


\section{References}

[1] Wikipedia. [Online]. Available: http://en.wikipedia.org/wiki/ASME_Boiler_ and_Pressure_Vessel_Code_(BPVC)

[2] G. Box, Statistics as a Catalyst to Learning by Scientific Method Part I-An Example, Jan,31 1999,ABI/INFORM Global.

[3] A. J. Keane and P. B. Nair, "Computational approaches for aerospace design," June 2005. DOI: 10.1002/0470855487.

[4] J. A. Goldak, "A direct search computational weld mechanics optimization using least square approximation," Proceedings of the ASME 2012 Pressure Vessels 83 Piping Conference, PVP2011.

[5] J. Goldak, "Framework for designer driven exploration of computational weld mechanics design space," Proceedings of the ASME 2012 Pressure Vessels 83 Piping Conference, PVP2011.

[6] K. Masabuchi, "Analysis of welded structures," MIT USA, Pergomon Press, International Series on Materials Science and Technology, vol. 33, pp. 172-187, 1980. 
[7] J.Bree, "The journal of strain analysis for engineering design," 1967. 226-38.

[8] S. Jahanian, "Thermoelastoplastic stree analysis of thick-walled tube of nonlinear strain hardening," ASME, 1996.

[9] A. Ponter, Shakedown and limit analyses for 3D structures using the linear matching method, 2001.

[10] M. Engelhardt, "Limit analysis for a general class of yield conditions," vol. 19, pp. 1-21, 2000.

[11] J. Corum, "Future needs for inelastic analysis in design of high-temperature nuclear plant components," vol. 13, pp. 231-240, 1981.

[12] M. Martin, "Application of direct cyclic analysis to the prediction of plastic shakedown of nuclear power plant components," 2008. 61067.

[13] S. Radoslav, User's Design Specification Preparation for 2Cr-1Mo-V Reactors in Accordance With ASME Section VIII, Division 2 Code and Code Case 2605, PVP 2013.

[14] R. A. Ainsworth, "Assessment procedure for the high temperature response of structures," 2003.

[15] H. Chen, The linear matching method applied to the high temperature life integrity of structures. Part 1. Assessments involving constant residual stress fields, 2006, vol. 83 . 
[16] H. F. Chen, The linear matchingmethod applied to the high temperature life integrity structures. Part 2. Assessments involving changing residual stress fields. Int J Pressure Vessels Piping, November 2005.

[17] M. Engelhardt, "Computational modelling of shakedown," PhD thesis. University of Leicester, 1999.

[18] A. Zakharchenko., "Development of a new methodology for the design and optimization of piping systems." Master thesis, Carleton University. 2000.

[19] O. Zienkiewicz, The Finite Element Method, University of Wales, McGRAWHILL Book Company (UC) Limited, 1977.

[20] Nakatsuka, "Startup thermal considerations forsupercritical-pressure light watercooled reactors," p. 221230, J. Nucl. Sci. Technol. 134 (3).

[21] Sutanto, "Time dependent start-up thermal analysis of a super fast reactor," Nuclear Engineering and Design.

[22] Q. Tanga, Various startup system designs of HPLWR and their thermal analysis.

[23] Y. I. TinTin YI, "Startup thermal analysis of a high-temperature supercriticalpressure light water reactor," Journal of Nuclear Science and Technology, pp. 790-801.

[24] I. Lee, Sampling-based RBDO using the stochastic sensitivity analysis and Dynamic Kriging method, 2011), vol. 44. 
[25] M. Schonlau, "Global versus local search in constrained optimization of computer models," New Developments and Applications in Experimental Design, vol. 34, pp. 11-25, 1998.

[26] D. R. JONES, "Efficient global optimization of expensive black-box functions," Journal of Global Optimization, vol. 13, p. 455492, 1998.

[27] ASME Boiler and Pressure Vessel Code Section III -Division 1 Subsection NB, ASME 2010.

[28] B. H. Dennis, Parallel thermo-elasticity optimization of 3D serpentine cooling passages in turbine blades, 2003.

[29] A. C. Timothy Gilman, Stress based environmental fatigue monitoring of PWR charching nozzle, PVP2013.

[30] M. Martin, "Large vessel nozzle penetration geometric optimization study in 3d incorporation design of experiments techniques," Proceedings of the ASME 2012 Pressure Vessels 83 Piping Conference, PVP2013.

[31] Taguchi, "The taguchi approach to parameter design," ASQC Congress Transactions, Anaheim, Calif., U.S.A, p. 168, 1986.

[32] B. Clemson, "Efficient methods for sensitivity analysis," p. 3149, 26 DEC 2006.

[33] Material library. VrSuite, 2014.

[34] M. A. Bhatti, Ed., Advanced Topics in Finite Element Analysis of Structures: With Mathematica and MATLAB Computations. John Wiley \& Sons Ltd, 2005, vol. 2 . 
[35] F. P. Incorpera, Ed., Fundamentals of Heat and Mass Transfer. John Wiley \& Sons Ltd, 2006. 